\title{
Measuring the Free Energy of Self-assembling Systems in Computer Simulation
}

\section{Dissertation}

zur Erlangung des Doktorgrades

der Mathematisch-Naturwissenschaftlichen Fakultäten der Georg-August-Universität zu Göttingen

vorgelegt von

Yuki Norizoe

aus Tokyo, Japan

Göttingen, 2010 
D 7

Referent:

Prof. Dr. Marcus Müller

Korreferent:

Prof. Dr. Thomas Pruschke

Tag der mündlichen Prüfung: 


\section{Abstract}

Membrane fusion is involved in a multitude of biological processes like endo- and exocytosis, viral infection or synaptic release. The detailed mechanism, however, is not well understood because the time and length scales - microseconds and tens of nanometers, respectively - make a direct experimental observation difficult. The study of this collective phenomena, involving many lipid molecules, is also difficult in simulations with atomistic resolution. While the details of the fusion pathways are still under debate, most fusion scenarios start with the formation of a stalk, which is a hour-glass shaped connection between the two apposing membranes that are going to fuse. Understanding the properties of this initial fusion intermediate is a key to controlling fusion of bilayer membranes.

We use a coarse-grained model for bilayer membranes. The lipid molecules are described by a simple bead-spring model and the solvent degrees of freedom are integrated out. The effective non-bonded interactions between the beads of the lipid molecules take the form of a virial expansion. Within the mean-field approximation, the coefficients of the expansion are related to the density and compressibility of the hydrophobic interior of the bilayer membrane and the repulsion between the hydrophilic and hydrophobic units. In order to employ such an excess free energy density functional for the non-bonded interactions in a particle-based simulation, the local densities are calculated from the explicit particle coordinates via a collocation lattice. This soft, solvent-free, coarse-grained model for bilayer membranes allows for an efficient simulation of membrane properties.

This coarse-grained model has been employed to study the excess free energy of stalks that form between apposing membranes as a function of the molecular asymmetry of the lipid molecules and the membrane tension. To this end, we have devised a general strategy for calculating free energies in self-assembling systems. The method relies on constructing a reversible thermodynamic path that connects the system of two apposed bilayers and the stalk configuration. This path is constructed in an extended state space using an inhomogeneous, external field that is designed to direct the assembly of the system into the two apposed membranes or the stalk structure in the absence of non-bonded interactions. Using expanded ensemble simulations it is demonstrated that the path is reversible and that the Helmholtz free energy can be obtained with high accuracy. Combining this result with grandcanonical simulations, we have determined the excess free energy of a stalk as a function of the membrane tension. 
In order to compute the dependence of the excess free energy of a stalk on the molecular architecture, we have used a semi-grandcanonical ensemble, where Monte Carlo moves mmutatellipids of one architecture into molecules with another architecture and vice versa. In this ensemble, the composition of the mixed bilayer membranes is controlled by the chemical potential difference between the species and we can compute the free energy change upon exchanging lipids with different architecture with relative ease.

With these computational techniques we systematically investigate the stability of the stalk structure. The simulations show that the excess free energy of stalks in on the order of $10 k_{B} T$, where $k_{B} T$ denotes the thermal energy unit. Stalks are comprised of a few tens of lipid molecules and the excess free energy increases with membrane tension. The stability of a stalk strongly depends on the molecular architecture. Amphiphiles with a large head groups give rise to highly metastable stalks, whereas very asymmetric amphiphiles can even reduce the excess free energy of the stalk to negative values, which correspond to a thermodynamically stable structure. 


\section{Zusammenfassung}

Die Fusion von Membranen ist Bestandteil einer Vielzahl von biologischen Prozessen, wie z.B. der Endo- und Exozytose, Virusinfektion oder synaptischen Ausschüttung. Der detaillierte Mechanismus ist jedoch nicht vollständig verstanden, weil die mesoskopischen Zeit- und Längenskalen von Mikrosekunden und einigen Nanometern eine direkte experimentelle Beobachtung erschweren. Die Untersuchung dieser kollektiven Prozesse, an denen viele Lipidmoleküle teilnehmen, ist auch für atomistische Simulationen schwierig. Während die Details des Fusionspfades immer noch strittig sind, beginnen die meisten Fusionsszenarien mit der Bildung einer sanduhrförmigen Verbindung (,Stalk”) zwischen den beiden gegenüberliegenden Membranen, welche fusionieren. Das Verständnis der Eigenschaften dieser ersten Übergangsstruktur ist ein Schlüssel zur Kontrolle der Fusion von Membranen.

Wir verwenden ein vergröbertes Modell für Doppelschichtmembranen. Die Lipidmoleküle werden durch ein einfaches Kugel-Feder-Modell beschrieben und die Freiheitsgrade des Lösungsmittels sind herausintegriert. Die effektiven, nichtgebundenen Wechselwirkungen zwischen den Segmenten der Lipidmoleküle haben die Form einer Virialentwicklung. Im Rahmen der Molekularfeldnäherung sind die Entwicklungskoeffizienten mit der Dichte und Kompressibilität des hydrophoben Membraninnerens und der Abstossung zwischen hydrophilien und hydrophoben Segmenten verknüpft. Um ein solches Dichtefunktional der ungebundenen Wechselwirkungen in Teilchensimulationen zu verwenden, werden die lokalen Dichten aus den expliziten Teilchenkoordinaten auf ein Gitter abgebildet. Dieses lösungsmittelfreie vergröberte Modell für Membranen mit weichen Wechselwirkungen erlaubt die effiziente Simulation von Membraneigenschaften.

Dieses vergröberte Modell wurde verwendet, um die freie Energie eines „Stalks” zwischen zwei gegenüberliegenden Membranen als Funktion der molekularen Asymmetrie der Lipide und der Membranspannung zu bestimmen. Zu diesem Zweck haben wir ein allgemeines Verfahren zur Berechnung von freien Energien in selbstordnenden Systemen entwickelt. Die Methode beruht auf einem thermodynamischen Pfad, welcher das System von zwei unverbundenen Membranen mit der ,Stalk”Struktur reversibel verbindet. Dieser Pfad verläuft in einem erweiterten Zustandsraum mit einem inhomogenen externen Feld. Dieses ist entworfen, um das System ohne ungebundene Wechselwirkungen zur Bildung der zwei gegenüberliegenden Membranen bzw. der „Stalk”-Struktur zu veranlassen. Mittels „expandedensemble"-Simulationen wird gezeigt, dass der Pfad tatsächlich reversibel ist und 
die Helmholtz'sche freie Energie wird mit großer Genauigkeit bestimmt. Durch zusätzliche großkanonische Simulationen haben wir die freie Energie des „Stalks” als Funktion der Membranspannung gemessen.

Um die Abhängigkeit der freien Energie von der molekularen Architektur zu berechnen, verwenden wir ein semi-großkanonisches Ensemble, in dem MonteCarlo-Schritte Lipide von einer Sorte in eine andere Lipidsorte mit verschiedener Architektur „mutieren” und umgekehrt. In diesem Ensemble wird die Zusammensetzung der Membran durch die Differenz der chemischen Potentiale der Spezies bestimmt und die Änderung der freien Energie bei dem Austausch einer Lipidsorte durch die andere kann leicht ermittelt werden.

Mit diesen Rechentechniken haben wir die Stabilität von „Stalks” systematisch untersucht. Die Simulationen zeigen, dass die freie Energie eines „Stalks” in der Größenordnung von etwa $10 k_{B} T$ liegt, wobei $k_{B} T$ die thermische Energieeinheit ist. „Stalks” bestehen aus 10-100 Lipidmolekülen und die freie Energie eines „Stalks” nimmt mit der Membranspannung zu. Die Stabilität hängt sehr stark von der Molekülarchitektur ab, Amphiphile mit grossen Kopfgruppen führen zu höchst metastabilen „Stalks”, während sehr asymmetrische Lipide die freie Energie von „Stalks” sogar zu negativen Werten verschieben, welche thermodynamisch stabilen Strukturen entsprechen. 


\section{Contents}

1 Introduction 1

2 Solvent-free model 9

2.1 Single component solvent-free homopolymers . . . . . . . . . . . 10

2.1.1 Free energy in solvent-free single component systems . . 10

2.1.2 Mean field approximation . . . . . . . . . . . . 11

2.1.3 Dimensionless parameters for solvent-free single component homopolymers . . . . . . . . . . . . . 12

2.1.4 Phase diagram for the single component solvent-free system 12

2.2 Solvent-free model of linear amphiphiles . . . . . . . . . . . . 13

2.3 Particle-Particle/Particle-Mesh (PPPM) method . . . . . . . . . . 14

2.3.1 Original PPPM method . . . . . . . . . . . . . . . . 15

2.3.2 PPPM improved with a linear interpolation . . . . . . . 16

2.3.3 Program test . . . . . . . . . . . . . . . 17

2.3 .4 Benchmark . . . . . . . . . . . . . . 20

2.4 Simulation of solvent-free homopolymers . . . . . . . . . 22

2.4.1 Locating the liquid-gas coexisting line . . . . . . . . 22

2.4.2 Simulation results of solvent-free homopolymers . . . . 23

3 Simulation method 29

$3.1 \quad$ NVT-Monte Carlo simulation technique . . . . . . . . . . . 29

3.1.1 Metropolis algorithm . . . . . . . . . . . . . . 30

3.1.2 Monte Carlo step (MCS) and acceptance ratio . . . . . . . 32

3.1.3 Mersenne Twister . . . . . . . . . . . . . . . . 32

3.2 Statistics for molecules with intra-structure . . . . . . . . . 33

3.2.1 Molecules with the internal structure . . . . . . . . . 33

3.2 .2 Ideal molecules . . . . . . . . . . . . . . . . . . . 33

3.2.3 Statistics of molecules in $\mu V T$-ensemble . . . . . . 37

3.3 Configurational-bias method in $\mu V T$-ensemble $\ldots \ldots \ldots . \ldots 38$

3.3.1 Particle insertion . . . . . . . . . . . . . . . 39

3.3.2 Detailed balance condition of the configurational-bias method 41

3.3.3 Test simulation of the configurational-bias method . . . 44

3.4 Chemical potential in NVT-ensemble . . . . . . . . . . 50 
3.4.1 Widom's insertion and deletion methods . . . . . . . . . 50

3.4.2 Chemical potential calculation via Rosenbluth sampling . 53

3.4 .3 Total chemical potential . . . . . . . . . . 55

$4 \quad$ Free energy calculation $\quad 57$

$4.1 \quad$ Free energy of crystals . . . . . . . . . . . . . . 57

4.2 Helmholtz free energy calculation based on an external field . . . 58

4.2.1 Molecules in the external field . . . . . . . . . . 58

4.2.2 Thermodynamic integration based on an external field . . 59

4.2.3 Method of expanded ensembles . . . . . . . . . . 61

4.2.4 Determining the external field . . . . . . . . . . . 63

4.3 Grand potential calculation . . . . . . . . . . . . . . 63

4.4 Semigrand canonical ensemble . . . . . . . . . . . . . 65

4.4.1 Statistics of semigrand canonical ensemble . . . . . . . 65

4.4.2 Semigrand canonical ensemble simulation . . . . . . . 67

4.4.3 Free energy difference via semigrand canonical simulation 67

4.4.4 Test simulation in semigrand canonical ensemble . . . . . 68

5 Simulation of bilayer membranes $\quad 73$

5.1 Simulation results of tensionless bilayers . . . . . . . . . 73

5.1.1 Tensionless bilayers at $N_{A}=28 \ldots \ldots \ldots \ldots$

5.1.2 Tensionless bilayers at $N_{A}=29$ and $27 \ldots \ldots \ldots$

5.2 Helmholtz free energy of the stalk at $N_{A}=28 \ldots \ldots \ldots$

5.2.1 External field calculation . . . . . . . . . . . 80

5.2.2 Expanded ensemble simulations at $N_{A}=28 \ldots \ldots$. . 80

5.2.3 Helmholtz free energy difference along each branch . . . . 81

5.3 Helmholtz free energy of the stalk at $N_{A}=29 \ldots \ldots$. . . . . 85

5.4 Helmholtz free energy of the stalk at $N_{A}=27 \ldots \ldots \ldots$. . . . 86

5.5 Grand potential of the stalk . . . . . . . . . . 90

6 Conclusions $\quad 95$

A Gaussian chain $\quad 99$

A.1 Microscopic polymer structure . . . . . . . . . . . . . . . . . . . . 99

A.2 Gaussian polymer chain . . . . . . . . . . . . . 102

A.2.1 Persistence length . . . . . . . . . . . . . . . . . . . 102

A.2.2 End-to-end vector . . . . . . . . . . . . . . . . 102

A.2.3 Gaussian chain . . . . . . . . . . . . . . . . . 104

A.2.4 Size of Gaussian chain . . . . . . . . . . . . . . . 104

A.2.5 Derivation of Gaussian chain . . . . . . . . . . . . 105

$\begin{array}{ll}\text { B Particle deletion } & 109\end{array}$

$\begin{array}{ll}\text { Bibliography } & 111\end{array}$ 
CONTENTS

Acknowledgements 117

Zusammenfassung

Erklärung

121

Lebenslauf

125 


\section{List of Figures}

1.1 Chemical structure of sphingosine $\ldots \ldots \ldots \ldots$

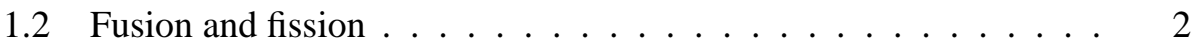

1.3 Classical bilayer membrane fusion process . . . . . . . . . . 3

1.4 Electron density map of a stalk derived from x-ray diffraction . . . 4

1.5 A phase diagram of the membrane fusion process. . . . . . . . 5

2.1 Phase diagram of solvent-free single component linear homopolymers constructed via mean-field approximation $\ldots \ldots \ldots 12$

2.2 Snapshot of diblock copolymers at $\chi N=16.0 \ldots \ldots \ldots \ldots$

2.3 Distribution of A-segments for diblock copolymers . . . . . . . 19

2.4 Distribution of A-segments for homopolymer blend . . . . . . . 19

2.5 Snapshots of the system at $\rho_{p}^{\prime} / \rho_{p c}^{\prime}=10.0$ and $3 \times 10^{6} \mathrm{MCS} \ldots .24$

2.6 Density profile along $z$-axis at $\rho_{p}^{\prime} / \rho_{p c}^{\prime}=10.0 \ldots \ldots \ldots 24$

2.7 Binodal lines calculated both via mean field approximation and via Monte Carlo simulation . . . . . . . . . . . . . . . 25

2.8 Snapshot of the system at $\rho_{p}^{\prime} / \rho_{p c}^{\prime}=1.0$ and $v^{\prime} / v_{c}^{\prime}=0.1$, taken at

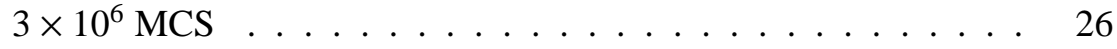

2.9 Density profile of the segments per cell at $\rho_{p}^{\prime} / \rho_{p c}^{\prime}=1.0 \ldots \ldots 26$

2.10 Density profile of the segments per cell _ . . . . . . . . 27

2.11 Density profile of the segments per cell at $\rho_{p}^{\prime} / \rho_{p c}^{\prime}=100.0$ and

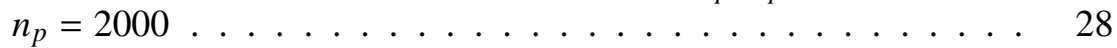

3.1 An example of a molecular configuration schematically represented by bond vectors . . . . . . . . . . . . . . . . 36

3.2 Step 2) in the particle insertion . . . . . . . . . . . . 41

3.3 The number of polymers sampled every MCS . . . . . . . . . 45

3.4 The number of polymers at $\rho_{\text {res }}^{\prime}=51$ sampled every MCS . . . . 47

3.5 The number of polymers at $\rho_{\text {res }}^{\prime}=171$ sampled every MCS $\ldots . \quad 47$

3.6 The number of polymers at $\rho_{\text {res }}^{\prime}=2646$ sampled every MCS $\ldots 48$

3.7 Probability density of $n_{p}$ at $\rho_{\text {res }}^{\prime}=171$ and $200 \ldots \ldots \ldots$

4.1 Triangular thermodynamic integration path . . . . . . . 60

4.2 Thermodynamic integration paths via semigrand canonical ensemble 69

$4.3\left(n_{p \alpha} / n_{p}\right)$ vs chemical potential difference between $\alpha$ and $\gamma \ldots 69$ 
4.4 Snapshots of the system at $7 \times 10^{5}$ MCS in semigrand canonical

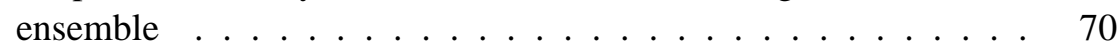

4.5 Probability densities of $n_{p \alpha}$ at $\Delta \mu:=\mu_{\alpha}-\mu_{\gamma}=0.3053 k_{B} T$ and

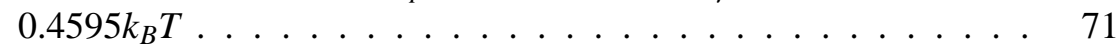

5.1 Molecular density profile across a tensionless bilayer . . . . . 74

5.2 Mean square displacement of one molecule at $N_{A}=28$ in the single tensionless bilayer . . . . . . . . . . . . . . 75

5.3 Chemical potential calculation of a tensionless bilayer at $N_{A}=28 \quad 76$

5.4 Thermodynamic integration paths for the Helmholtz free energy calculation at $N_{A}=28 \ldots \ldots \ldots \ldots \ldots$

5.5 2D contour plots of the molecular distribution around the stalk . . 81

5.6 Time evolution of $\lambda_{E}$ in the middle of the expanded ensemble sim-

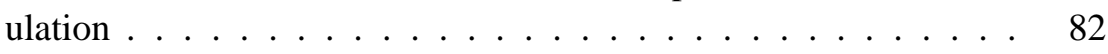

5.7 Thermodynamic integrands on the branches $1 \rightarrow 2$ and $4 \rightarrow 5 \ldots 83$

5.8 Thermodynamic integrands on the branches $2 \rightarrow 3$ and $3 \rightarrow 4 \quad \ldots \quad 84$

5.9 Thermodynamic integrands on the branch $2 \rightarrow 4 \ldots \ldots$. . . 84

5.10 Thermodynamic integration paths for the Helmholtz free energy calculation at $N_{A}=29 \ldots \ldots \ldots \ldots$

5.11 Semigrand canonical ensemble simulation results between $N_{A}=$ 29 and $N_{A}=28 \ldots \ldots \ldots \ldots \ldots \ldots \ldots$

5.12 Semigrand canonical ensemble simulation results between $N_{A}=$ 29 and $N_{A}=28 \ldots \ldots \ldots \ldots \ldots \ldots \ldots \ldots \ldots$

5.13 Semigrand canonical ensemble simulation results between $N_{A}=$ 28 and $N_{A}=27 \ldots \ldots \ldots \ldots \ldots \ldots \ldots$

5.14 Number of molecules per area in the grand canonical ensemble at

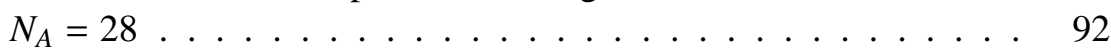

5.15 Excess grandcanonical potential of the stalk at $N_{A}=28 \ldots \ldots 2$

5.16 Increase in molecular density distribution of tails in stalk morphology with $\mu$ at $N_{A}=28 \ldots \ldots \ldots \ldots \ldots$

5.17 Number of molecules per area in the grand canonical ensemble at $N_{A}=29 \ldots \ldots \ldots \ldots \ldots \ldots \ldots$

5.18 Excess grandcanonical potential of the stalk at $N_{A}=29 \ldots \ldots 94$

A.1 Ethylene and polyethylene . . . . . . . . . . . . . . 99

A.2 Steric structure of the carbon backbone in a linear polymer on an atomic scale . . . . . . . . . . . . . . . 100

A.3 Energy between the carbon atoms as a function of internal rotation angle $\phi \ldots \ldots \ldots \ldots \ldots 1 . \ldots \ldots 1$

A.4 Planar trans-zigzag conformation . . . . . . . . . . . . . . . . 101

A.5 Polymer conformation on a large scale . . . . . . . . . . . 101

A.6 Linear ideal polymer . . . . . . . . . . . . . . . . . . 103

A.7 Complex integration paths for Gaussian chain . . . . . . . . 106 


\section{LIST OF FIGURES}

B.1 Step 2) in the particle deletion . . . . . . . . . . . 110 


\section{List of Tables}

2.1 Virial coefficients for solvent-free linear amphiphiles . . . . . . 15

2.2 Simulation parameters for benchmark on 1:1 A-B monomer blend 20

2.3 Computational time for $1: 1$ A-B monomer blend . . . . . . . . 20

2.4 Simulation parameters for benchmark on A-B diblock copolymers 21

2.5 Computational time for A-B diblock copolymers . . . . . . . . 21

2.6 Simulation parameters in the single component solvent-free homopolymers . . . . . . . . . . . . . . . 22

3.1 Simulation parameters for the test simulation of single-component solvent-free homopolymers on configurational-bias method in grand canonical ensemble . . . . . . . . . . . . . . . 46

$3.2 \rho_{\text {res }}^{\prime}$ and corresponding $\rho_{p}^{\prime}$ via mean-field approximation $\ldots \ldots .47$

5.1 Fixed simulation parameters for the solvent-free lipid model . . . 73

5.2 System size and $n_{p}$ for the Helmholtz free energy calculation $\ldots 80$

5.3 Helmholtz free energy difference on each branch at $N_{A}=28 \ldots 83$

5.4 Chemical potentials on each state at $N_{A}=28 \ldots \ldots \ldots$. . . . 91

5.5 Chemical potentials on each state at $N_{A}=29 \ldots \ldots \ldots$. . . . 91

A.1 Complex integration paths for Gaussian chain $\ldots \ldots \ldots$ 


\section{Chapter 1}

\section{Introduction}

We study transition states of bilayer membrane fusion by Monte Carlo simulation using a solvent-free model [1]. Figure 1.1 presents the chemical structure of a sphingosine as a particular example of lipid molecules. A lipid molecule, also called a lipid, is an amphiphilic molecule composed of a hydrophilic block, called a head, and a hydrophobic block, a tail. In an aqueous solution, the tails of many amphiphilic molecules gather into one planar structure, i.e. a membrane, to reduce the interface between water and the tails. The heads are excluded from the inside of the membrane, i.e. repelled from the dense tails, and collected on the surfaces of the membrane. This structure is called a bilayer membrane. Cell membranes, an example of biological membranes, belong to this class of morphology.

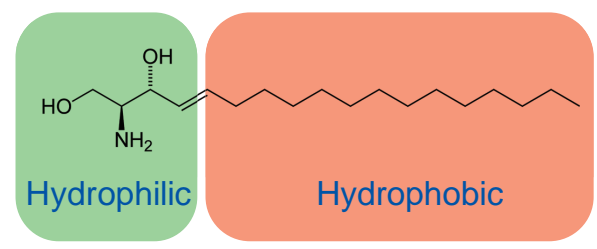

Figure 1.1: Chemical structure of sphingosine, a lipid molecule, with molecular formula $\mathrm{C}_{18} \mathrm{H}_{37} \mathrm{NO}_{2}$. A lipid is divided in hydrophilic block, called heads, and hydrophobic block, denoted as tail.

Interestingly, bilayer structures are also observed in another class of assembling systems: the solutions of amphiphilic block copolymer molecules [2-5]. The similarities of these systems to lipids does not remain only on the level of comparable self organization patterns. They exhibit also similar dynamical phenomena such as fusion and fission of the bilayer membranes [6]. An example of these phenomena observed in the case of polymeric vesicles (i.e. spherical bilayer membranes) is shown in Fig. 1.2. This universal behavior, demonstrated by amphiphilic systems with very different chemical structure, should be attributed to a generic feature characterizing these systems: the connectivity of hydrophylic and hydrophobic parts. This observed universality has a very important consequence: it suggests that a qualitative understanding of the behavior of the amphiphilic sys- 
tems on mesoscale can be obtained within the framework of simple minimal models, incoorporating only the relevant interactions that are necessary to reproduce the phenomena of interest [7]. By this way, incoorporating atomistic details can be avoided, and the collective phenomena (i.e. processes involving a large number of amphiphilic molecules) can be addressed within computationally efficient coarse-grained simulation techniques.
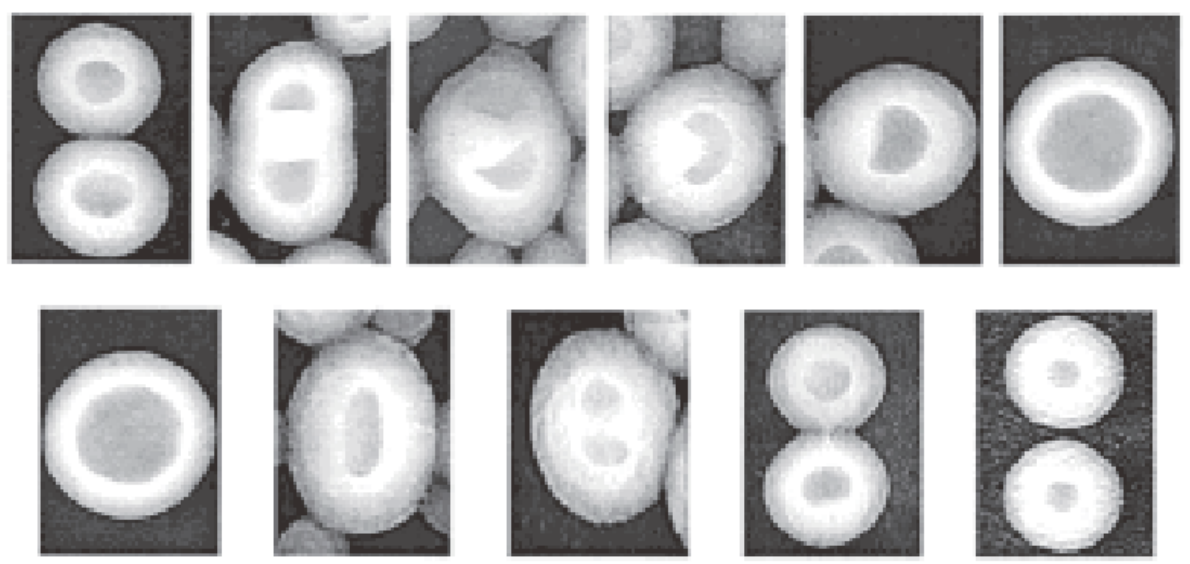

Figure 1.2: Vesicle fusion, shown in the first row, and vesicle fission, in the bottom row (from Ref. [6]); an analogy of similar processes observed in biological lipid membranes.

The extent of the decimation of the degrees of freedom in coarse-grained models is closely related to the phenomena one would like to address. In this scope, a frequent approach when studying structure formation and evolution in amphiphilic membranes, is to describe these systems within implicit solvent models. In this description the solvent molecules are "integrated out" and the solvent presence is taken effectively into account by proper selection of the interaction potentials between the beads of the coarse-grained amphiphiles. An early example of such an approach is presented in the work of Drouffe et al [8]. These authors studied the assembly of amphiphilic vesicles by representing the amphiphiles with spheres interacting with a combination of hard core repulsions, orientation-dependent potentials, and multibody interactions. The multibody character of the interactions had to be introduced to mimic the "hydrophobic effect" so that a stable, fluid "membrane" in the form of a single monolayer of spheres formed at high temperatures. A more realistic solvent-free minimal model of amphiphilic bilayers was introduced by Noguchi and Takasu [9],followed by Farago [10] and Cooke and Deserno [11, 12]. One of the main drawbacks of these models is the rather "ad-hoc" choice of the interaction potential, which cannot be connected in a straightforward way to the thermodynamic and the mechanical properties of the membrane.

In our work we present a solvent-free model based on a density functional description $[1,13]$ of the amphiphilic membranes. The parameters of the model can be connected in a straightforward way to thermodynamic properties such as the density of the bilayer, its compressibility and incompatibility between the hy- 
drophobic/hydrophilic beads. The density-functional based representation serves as a basis for a Monte Carlo simulation scheme [14-17]. Essentially, the DFTinteractions are equivalent to soft intermolecular potentials similar to those that are used in dissipative particle dynamics (DPD) [18-20]. Unlike DPD, however, the present model facilitates the simulation due to an absence of explicit solvent molecules. In this work, the DFT-based Monte Carlo technique will be used to study the process of membrane fusion via the free energy of an important intermediate structure.

Fusion of bilayer membranes is an event essential in biological systems, e.g. viral infection, synaptic release, and endocytosis [21-24]. At the initial stages of the fusion, the isolated membranes are brought into proximity controlled by proteins (e.g. SNARE protein family [25]). After the membranes are approaching, fusion events alter the membrane topology. This is a collective phenomenon, in which a large number of molecules in the membranes participate. In phenomenological theories, intermediate structures along the fusion process are assumed, as is illustrated in Fig. 1.3. Among these intermediate structures, the stalk morphology plays a vital role in the fusion process. This morphology is the initial intermediate that is formed by an hour-glass shaped connection between the two apposing membranes. The evolution pathways through which the stalk evolves into the fusion pore are still under debate [26].

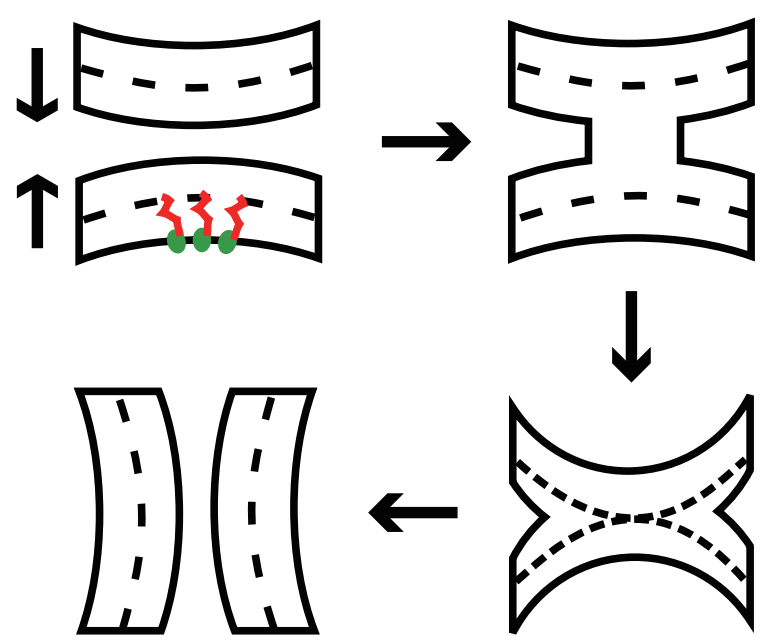

Figure 1.3: Classical bilayer membrane fusion process. First a pair of apposed bilayers forms the stalk, an hourglass-shaped structure, that bridges these bilayers. From this structure, they start the fusion and form, via the hemifusion diafragm, the final fusion pore. Arrows show the time order of these intermediate states. Hydrophobic tails and hydrophilic heads of amphiphiles are drawn in red and green respectively.

The stability of the stalk itself will depend on specific properties of the amphiphilic system. To this end one can encounter cases of highly unstable stalks as well as systems where the bilayers are bridged by dense arrays of stalks. The latter case has been experimentally observed in aqueous solutions of lipids $[27,28]$ and 
diblock copolymer melts [29]. Figure 1.4 shows an example of an electron density map of such a stable stalk structure derived from x-ray diffraction for lipid bilayer membranes.

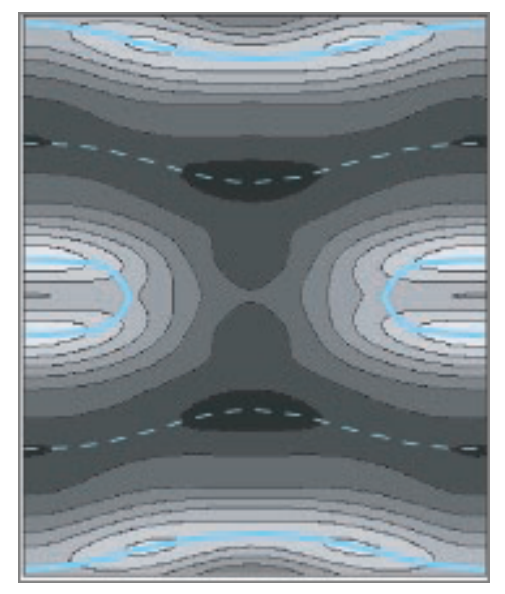

Figure 1.4: Electron density map of a stalk derived from x-ray diffraction patterns observed in lipid bilayer membranes [27]. These bilayers form stable stalk structures arranged on a hexagonal lattice. The bilayer membrane fusion process stops at this state in this experiment.

From numerical self-consistent field calculations [30,31], it is known that membrane fusion is observed only in a small region of molecular asymmetry and membrane tension according to the stability of the stalk, as is shown in Fig. 1.5. In particular, when the amphiphilic molecules are too symmetric, the stalk structure, from which the fusion always starts, is not even metastable and the membrane fusion process proceeds very slowly. On the other hand, when the amphiphilic molecular asymmetry is too high, bilayers become unstable and the stalk spontaneously expands into an elongated shape. The membrane tension plays also a significant role in stalk evolution promoting the opening of the fusion pore. Of course, when the membrane tension is too high, fusion competes with membrane rupture. These experimental and theoretical observations indicate that investigating the stalk stability, i.e. quantifying the excess free energy of the stalk, is a key to understanding and controlling membrane fusion and that the stability of the stalk structure depends on the molecular architecture and the membrane tension.

Early phenomenological theories estimated the excess free energy of the stalk, in other words, the free energy difference between the apposed bilayers and the stalk morphology, to be on the order of a few hundreds $k_{B} T$, where $k_{B} T$ denotes the thermal energy, an impractically large value for biological systems. An improved theoretical description [32], incorporating a more realistic representation of the stalk structure, significantly lowered this result to $30-40 k_{B} T$. Subsequently self-consistent field calculations $[30,31]$ have been used to determine the free energy of the stalk and other intermediate structures along the fusion pathway without assumptions about the detailed geometry and molecular conformations. 


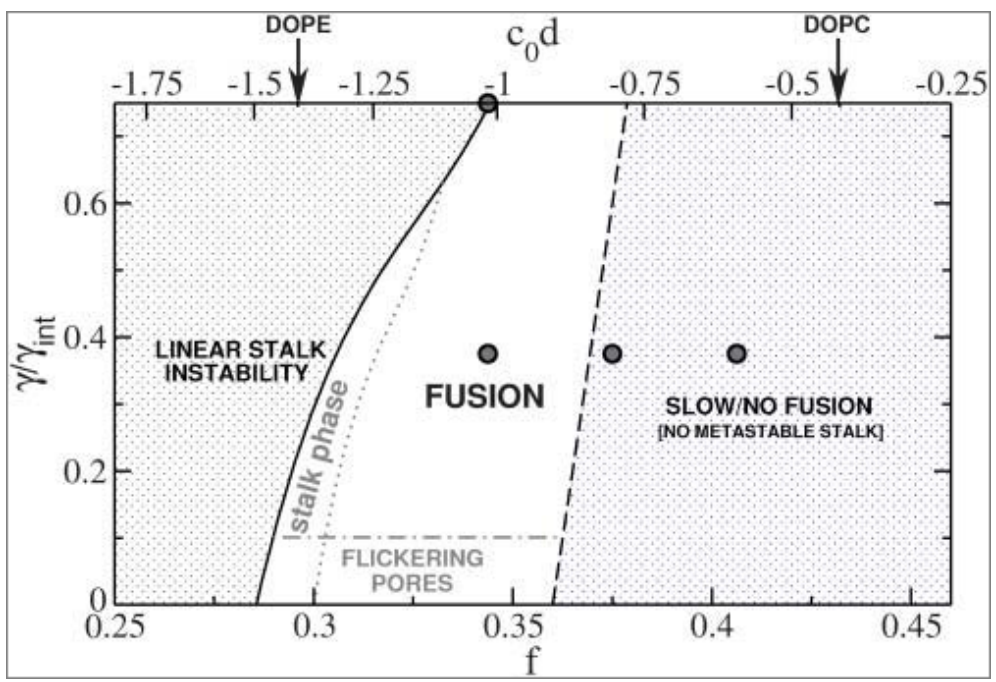

Figure 1.5: A phase diagram of the membrane fusion process by numerical self-consistent field calculations [30]. $f$ denotes the fraction of the hydrophilic head in one molecule and $\gamma$ the membrane tension. Within the unshaded region, the fusion process is successfully finished and the fusion pore appears.

These mean-field calculations have yielded an even lower excess free energy of the stalk [30,31], on the order of $13 k_{B} T$. The self-consistent field calculations, however, were limited to a random-walk-like molecular architecture (Gaussian chain model) and did not incorporate fluctuations. Both assumptions are appropriate for polymeric membranes but less so for lipid membranes. In the present dissertation, we determine the excess free energy of the stalk via molecular simulation method based on Monte Carlo simulations.

Generally calculating the excess free energy of self-assembled structures by computer simulation, however, is a challenge because the free energy of a system is not a simple function of the particle coordinates, and special simulation techniques have been devised [33]. In hard-condensed matter systems, e.g. crystals, one popular method consists in calculating the free energy by thermodynamic integration along a path that reversibly connects the structure of interest to a reference state of known free energy. For crystalline solids, the Einstein crystal is an appropriate reference state, where non-interacting particles are harmonically tethered to their ideal lattice position. The free energy of the ordered system is derived [34] from thermodynamic integration based on gradually decreasing the strength of the tethers and, in turn, increasing the interactions between particles. In selfassembling fluids, however, there is no analog of the Einstein crystal because even in the defect-free, self-assembled state molecules diffuse and are not constrained to be at some preferential positions; hence the above technique can not be easily generalised to particle-based simulations (cf. Ref. [35] for a field-theoretic approach).

An alternative technique [36,37], inspired by similar methods developed for crystalline solids [38-40], consists in calculating the free energy difference be- 
tween the structures of interest by transforming them reversibly into each other with the help of an external, ordering field. Like the transition from a liquid to a crystal, self-assembly or transformation between different morphologies in response to a physically relevant control parameter (e.g., temperature, density, or repulsion between amphiphilic entities) occur via first-order transitions. Using an external, ordering field, whose spatial structure and strengths are adopted to the self-assembled structure and varying the intermolecular interactions, one can avoid the first-order transition and transform one structure into another via a reversible path. For the self-assembly from a disordered structure of an ideal gas, such a transformation path is comprised of two branches: Along the first branch, one transforms the self-assembled system into an ideal gas that exhibits the same (or very similar) spatial organisation due to the presence of external, ordering fields. Along this branch, the intermolecular interactions are gradually decreased to zero while, simultaneously, the strength of the external, ordering field is increased such that the structural changes along this branch are minimised [38]. Optimally, the morphology remains unaltered during the entire transformation, therefore, this transformation is free of thermodynamic singularities, and the concomitant free energy difference between the self-assembled fluid and the ideal gas in the external fields can be obtained by thermodynamic integration. Along the second branch, we transform the externally structured, ideal gas into a disordered one by progressively reducing the strength of the auxiliary fields. This is also a reversible process because of the absence of collective, ordering effects in the non-interacting system, and the free energy difference along this branch can be obtained by thermodynamic integration (TDI). Along this transformation path one transforms a self-assembled fluid into an ideal gas without passing through a first-order transition.

In this dissertation we choose the second option and construct a reversible path that connects the configurations of two apposed bilayers reversible with the stalk structure with the help of a specifically disigned external field. Simulating the bilayers in our solvent-free model, we measure the excess free energy of the stalk via TDI based on the external field with an accuracy of $10^{-3} k_{B} T$ per molecule.

Experimental works [29] indicate that large molecular asymmetries, which correspond to the large tail blocks at the fixed molecular weight, increase the stability of the stalk. This is also shown by self-consistent field calculations [30]. In this work, we study this dependence of the stalk stability on the molecular architecture by determining the excess free energy of the stalk at various block ratios between the heads and the tails, and also the dependence on the membrane tension.

We begin our discussion with the solvent-free model in chapter 2 . Combined with PPPM method [41-43], our solvent-free model facilitates the Monte Carlo simulation. Details of the simulation methods are introduced in chapter 3. In addition to the conventional Metropolis algorithm, a configurational-bias method used for simulation in the grand canonical ensemble and for the chemical potential calculation is derived. Free energy calculation methods are discussed in chapter 4. Expanded ensemble simulations quantitatively verify the absence of a first-order transition along the path that reversibly connects two apposing bilayers with the stalk 
morphology. Via semigrand canonical ensemble, the Helmholtz free energy difference between single component systems composed of different molecular species is directly measured. Simulation results on the bilayer membranes are presented in chapter 5. The dependence of the excess free energy of the stalk on molecular architecture and the membrane tension is obtained. The number of molecules composing the stalk structure is analyzed in the grand canonical ensemble. We summarize our work in chapter 6 and provide an outlook on further applications. 


\section{Chapter 2}

\section{Solvent-free model}

Membranes are self-assembled structures that are composed of a large number of molecules. Even though industry keeps making improvement to our computers, we cannot simulate, with use of the atomistic simulation technique, phenomena found in macromolecular systems on large spatial and temporal scales on account of many degrees of freedom. Whereas these simulation methods in an atomistic scale yield correct data, we need to study coarse-grained simulation methods in a molecular level in order to reduce required computational time and reproduce large systems.

In conventional coarse-grained molecular simulation, due to a large number of particles within the cutoff distance of pair interaction potetnial, computational time increases at high density. PPPM techniques [41-43] discussed in section 2.3 solves this issue. Despite the efficiency of PPPM, however, countless solvent molecules that surround solute molecules still pose a computational challenge that cannot be treated within acceptable computational time.

For the sake of avoiding this difficulty, Drouffe, Maggs, and Leibler proposed a solvent-free model [8]. In their model solvent molecules are replaced with an effective interaction potential between solute molecules, i.e. implicit solvents, for the purpose of integrating out the explicit solvent. Their solvent-free model attained the efficiency which could not be reached by models including explicit solvent molecules. Other solvent-free models have also been invented [44].

Due to the absence of solvent molecules a solvent-free model cannot induce aggregation without attractive interaction force between particles. This is one of the significant properties of solvent-free model. For example, in dissipative particle dynamics (DPD) technique, particles interact only via repulsive pair interaction potential [20].

In the present dissertation, we present a solvent-free model based on virial expansion coupled with our improved PPPM method and study the phenomena observed in macromolecular systems on a large scale. The virial expansion yields a simple physical interpretation of the excess free energy functional of the nonbonded interactions compared to previous solvent-free models. 
The solvent-free model composed of single component homopolymers is discussed first. Next, we apply this simulation method to the solvent-free model of diblock copolymers studying bilayers.

\subsection{Single component solvent-free homopolymers}

We construct our single-component solvent-free linear homopolymer system based on the bead-spring model discussed in section A.2. Two types of interactions can be distinguished:

i) Bonded, intramolecular interactions characterize the molecular architecture and they represent the connectivity along the backbone of a macromolecule or a lipid.

ii) Non-bonded interactions characterize the repulsion between the amphiphilic units and they drive the self-assembly into bilayer membranes.

Our bonded potential between a pair of linked segments is given by a harmonic spring (see eq. (A.5)):

$$
\frac{H_{\text {spring }}}{k_{B} T}=\frac{3}{2} \frac{N-1}{R_{e}^{2}} r^{2}
$$

where $r$ is the distance between the linked segments and $N$ the polymerization degree. $R_{e}$, the end to end distance of an ideal linear polymer, is used as the unit of length scale (cf. eq. (A.6)).

\subsubsection{Free energy in solvent-free single component systems}

The non-bonded interactions are described by an excess free energy in our coarsegrained model.

Here we discuss our solvent-free single component homopolymers in the canonical ensemble. We assume that the total Helmholtz free energy of our system is defined as

$$
F\left(T, V, n_{p}\right):=F_{\text {ideal }}\left(T, V, n_{p}\right)+F_{\text {excess }}\left(T, V, n_{p}\right),
$$

where $n_{p}$ is the number of polymers and $V$ denotes the system volume.

The ideal part of the free energy is given in equations (3.22) and (3.24).

$$
\frac{F_{\text {ideal }}\left(T, V, n_{p}\right)}{k_{B} T}=-n_{p} \log \left(\frac{V e}{n_{p} \Lambda^{3}} Z_{0}\right)
$$

The phenomenalogical non-ideal part of free energy is defined via a virial expansion as

$$
\frac{F_{\text {excess }}\left(T, V, n_{p}\right)}{k_{B} T}:=\int_{V} d V\left(-\frac{1}{2} v \rho_{s}^{2}+\frac{1}{3} w \rho_{s}^{3}\right),
$$

where $\rho_{s}$ denotes the local number density of segments. Constant parameters $v>0$ and $w>0$, which correspond to the attractive and repulsive interaction strength 
between the segments, respectively. At large $v$ and finite $w$, the polymers aggregate into one dense droplet floating in a vacuum, i.e. macro-phase separation, whereas at finite $v$ and extremely high $w$ the system is in homogeneous phase.

\subsubsection{Mean field approximation}

We can investigate the phase behavior of our system using the mean field approximation [45]. In the framework of the mean field theory we assume that the number density of the segments $\rho_{s}$ is homogeneous. This yields the excess free energy

$$
\frac{F_{\mathrm{excess}}\left(T, V, n_{p}\right)}{k_{B} T} \approx V\left(-\frac{1}{2} v \rho_{s}^{2}+\frac{1}{3} w \rho_{s}^{3}\right)
$$

Therefore the total free energy becomes

$$
f\left(\rho_{s}\right):=\frac{F\left(T, V, n_{p}\right)}{V k_{B} T} \approx-\frac{\rho_{s}}{N} \log \left(\frac{N e Z_{0}}{\rho_{s} \Lambda^{3}}\right)-\frac{1}{2} v \rho_{s}^{2}+\frac{1}{3} w \rho_{s}^{3}
$$

The critical point on the $\rho_{s}-v$ phase diagram (see section 2.1.4 and Fig. 2.1), $\left(\rho_{s c}, v_{c}\right)$, of our system is determined from the conditions

$$
\begin{aligned}
& \frac{d^{2}}{d \rho_{s}^{2}} \frac{F\left(T, V, n_{p}\right)}{V k_{B} T}=\frac{1}{N \rho_{s}}-v+2 w \rho_{s}=0 \\
& \frac{d^{3}}{d \rho_{s}^{3}} \frac{F\left(T, V, n_{p}\right)}{V k_{B} T}=-\frac{1}{N} \rho_{s}^{-2}+2 w=0 .
\end{aligned}
$$

We find that the critical point is placed at

$$
\left(\rho_{s c}=\frac{1}{\sqrt{2 N w}}, v_{c}=2 \sqrt{\frac{2 w}{N}}\right) .
$$

The binodal line in our phase diagram (see Fig. 2.1) is determined from the simultaneous conditions

$$
\left.\frac{d f\left(\rho_{s}\right)}{d \rho_{s}}\right|_{\rho_{s}=\rho_{s 0}}=\left.\frac{d f\left(\rho_{s}\right)}{d \rho_{s}}\right|_{\rho_{s}=\rho_{s 1}}=\frac{f\left(\rho_{s 1}\right)-f\left(\rho_{s 0}\right)}{\rho_{s 1}-\rho_{s 0}},
$$

where the chemical potential is given by

$$
\frac{d f\left(\rho_{s}\right)}{d \rho_{s}}=\frac{1}{N}\left(\log \left(\frac{\rho_{s} \Lambda^{3}}{N e Z_{0}}\right)+1\right)-v \rho_{s}+w \rho_{s}^{2}
$$

and the system is separated into the two phases, a gas and a dense liquid, $\rho_{s}=\rho_{s 0}$ and $\rho_{s}=\rho_{s 1}$. 
Binodal line determined numerically from mean field approximation

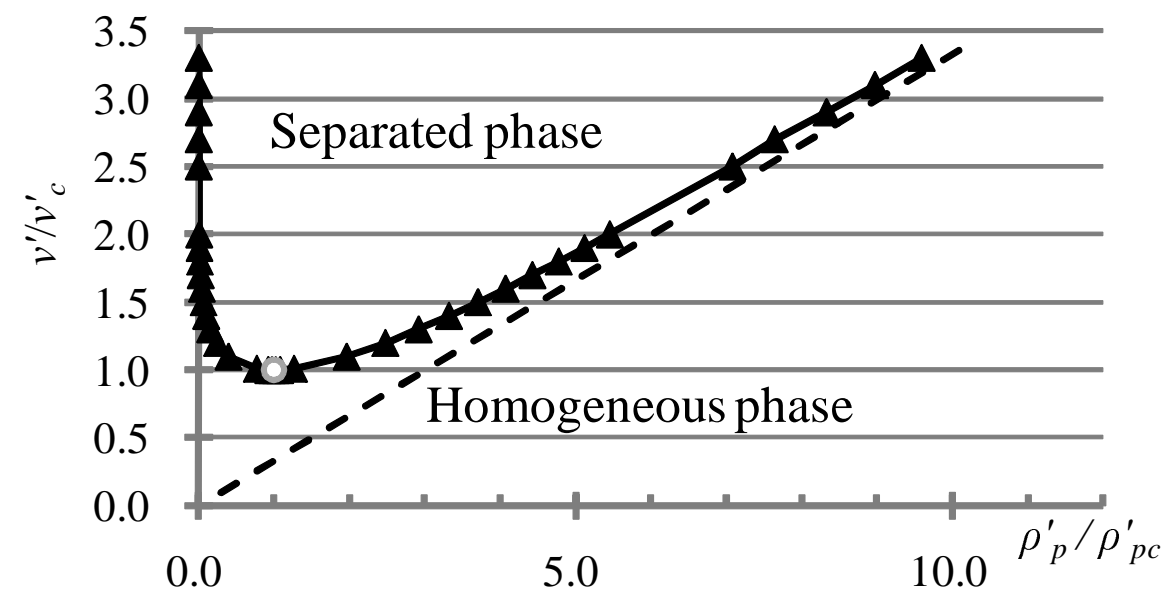

Figure 2.1: Phase diagram of solvent-free single component linear homopolymer system numerically constructed from simultaneous equations (2.12) and (2.13). The critical point is pointed by a circle. The broken line shows the asymptotic behavior of the binodal line given in eq. (2.17) at high density. $w^{\prime}$ is fixed at 1.0.

\subsubsection{Dimensionless parameters for solvent-free single component ho- mopolymers}

In our calculations we use dimensionless units. The dimensionless polymer density is defined as $\rho_{p}^{\prime}=\rho_{s} R_{e}^{3} / N$, the dimensionless parameter $w^{\prime}=w N^{3} / R_{e}{ }^{6}$, and the dimensionless parameter $v^{\prime}=v N^{2} / R_{e}^{3}$. With these dimensionless parameters, the critical point (2.7) is located on $\rho_{p c}^{\prime}=\rho_{s c} R_{e}^{3} / N$ and $v_{c}^{\prime}=v_{c} N^{2} / R_{e}{ }^{3}$,

$$
\left(\rho_{p c}^{\prime}=\frac{1}{\sqrt{2 w^{\prime}}}, v_{c}^{\prime}=2 \sqrt{2 w^{\prime}}\right)
$$

and the non-ideal part of the free energy (2.3) is represented by the dimensionless expression

$$
\frac{F_{\text {excess }}\left(T, V, n_{p}\right)}{k_{B} T}=\int_{V} \frac{d V}{R_{e}{ }^{3}}\left(-\frac{1}{2} v^{\prime} \rho_{p}^{\prime 2}+\frac{1}{3} w^{\prime} \rho_{p}^{\prime 3}\right)
$$

\subsubsection{Phase diagram for the single component solvent-free system}

The binodal line in our phase diagram can be calculated from the equations (2.8) which are based on the mean field approximation. With our dimensionless parameters defined in section 2.1.3, this condition is changed into the dimensionless 
equations

$$
\begin{aligned}
\rho_{p 1}^{\prime}\left\{\log \left(\frac{\rho_{p 0}^{\prime}}{\rho_{p 1}^{\prime}}\right)+1\right\}-\rho_{p 0}^{\prime} & +v^{\prime}\left(-\rho_{p 1}^{\prime} \rho_{p 0}^{\prime}+\frac{1}{2} \rho_{p 1}^{\prime}{ }^{2}+\frac{1}{2} \rho_{p 0}^{\prime}{ }^{2}\right) \\
& +w^{\prime}\left(\rho_{p 1}^{\prime} \rho_{p 0}^{\prime}{ }^{2}-\frac{2}{3} \rho_{p 0}^{\prime}{ }^{3}-\frac{1}{3} \rho_{p 1}^{\prime}{ }^{3}\right)=0
\end{aligned}
$$

and

$$
\log \left(\frac{\rho_{p 0}^{\prime}}{\rho_{p 1}^{\prime}}\right)+v^{\prime}\left(\rho_{p 1}^{\prime}-\rho_{p 0}^{\prime}\right)+w^{\prime}\left(\rho_{p 0}^{\prime 2}-\rho_{p 1}^{\prime}{ }^{2}\right)=0,
$$

where the system is separated into the two phases $\rho_{p}^{\prime}=\rho_{p 0}^{\prime}$ and $\rho_{p}^{\prime}=\rho_{p 1}^{\prime}$.

In the dense liquid $\rho_{p 1}^{\prime} \gg \rho_{p 0}^{\prime}$, these two equations are reduced to

$$
\log \left(\frac{\rho_{p 0}^{\prime}}{\rho_{p 1}^{\prime}}\right)+1+\frac{1}{2} v^{\prime} \rho_{p 1}^{\prime}-\frac{1}{3} w^{\prime} \rho_{p 1}^{\prime}{ }^{2}=0
$$

and

$$
\log \left(\frac{\rho_{p 0}^{\prime}}{\rho_{p 1}^{\prime}}\right)+v^{\prime} \rho_{p 1}^{\prime}-w^{\prime} \rho_{p 1}^{\prime}{ }^{2}=0 .
$$

We can exactly solve these reduced simultaneous equations (2.14) and (2.15) and determine the binodal line,

$$
\rho_{p 1}^{\prime}=\frac{\frac{3 v^{\prime}}{4 w^{\prime}}+\sqrt{\left(\frac{3 v^{\prime}}{4 w^{\prime}}\right)^{2}-\frac{6}{w^{\prime}}}}{2}
$$

At $v^{\prime 2} \gg w^{\prime}$, this solution shows the linear relation between $\rho_{p 1}^{\prime}$ and $v^{\prime}$,

$$
\rho_{p 1}^{\prime} \cong \frac{3 v^{\prime}}{4 w^{\prime}} .
$$

In this limit, a dense polymer melt (i.e. the interior of a lipid bilayer) coexists with a vapor of vanishingly small density. The coexistence pressure is virtually zero.

In the intermediate density regions, solving numerically the two simultaneous equations (2.12) and (2.13) with the two parameters $\rho_{p 0}^{\prime}$ and $\rho_{p 1}^{\prime}$, we can obtain the prediction of the binodal line shown in Fig. 2.1. Note that at this density the pressure vanishes.

\subsection{Solvent-free model of linear amphiphiles}

We apply the solvent-free single component homopolymer model to linear amphiphilic molecules composed of an A-block and a B-block. Segments are bonded 
by the harmonic springs given in eq. (2.1). For these amphiphilic molecules, $F_{\text {excess }}\left(T, V, n_{p}\right)$ is defined, in terms of the reduced dimensionless parameters, as

$$
\begin{aligned}
& \frac{F_{\text {excess }}\left(T, V, n_{p}\right)}{k_{B} T} \\
& =\int_{V} \frac{d V}{R_{e}^{3}}\left\{-\frac{1}{2} v_{\mathrm{AA}}^{\prime} \rho_{p \mathrm{~A}}^{\prime 2}-\frac{1}{2} v_{\mathrm{BB}}^{\prime} \rho_{p \mathrm{~B}}^{\prime 2}+v_{\mathrm{AB}}^{\prime} \rho_{p \mathrm{~A}}^{\prime} \rho_{p \mathrm{~B}}^{\prime}\right. \\
& \left.\quad+\frac{1}{3}\left(w_{\mathrm{AAA}}^{\prime} \rho_{p \mathrm{~A}}^{\prime 3}+3 w_{\mathrm{AAB}}^{\prime} \rho_{p \mathrm{~A}}^{\prime 2} \rho_{p \mathrm{~B}}^{\prime}+3 w_{\mathrm{ABB}}^{\prime} \rho_{p \mathrm{~A}}^{\prime} \rho_{p \mathrm{~B}}^{\prime 2}+w_{\mathrm{BBB}}^{\prime} \rho_{p \mathrm{~B}}^{\prime 3}\right)\right\}
\end{aligned}
$$

where

$$
\begin{aligned}
\rho_{p \omega}^{\prime} & :=\frac{\rho_{s \omega} R_{e}{ }^{3}}{N} \\
v_{\omega \eta}^{\prime} & :=\frac{v_{\omega \eta} N^{2}}{R_{e}{ }^{3}} \\
w_{\omega \eta \tau}^{\prime} & :=\frac{w_{\omega \eta \tau} N^{3}}{R_{e}{ }^{6}},
\end{aligned}
$$

$\omega, \eta$, and $\tau$ denote the segment species i.e. A or $\mathrm{B}, \rho_{s \omega}$ is the local segment density of $\omega$-species, and $\rho_{p \omega}^{\prime}$ dimensionless molecular density of $\omega$-species. $v_{\omega \eta}$ and $w_{\omega \eta \tau}$ are the 2 nd and the 3 rd virial coefficients respectively.

The virial coefficients used in the present thesis are shown in Table 2.1. With these interaction parameters, our solvent-free linear amphiphilic molecules form a stable bilayer structure, which is simulated in chapter 5. The A-block and the B-block correspond to the hydrophobic tail and the hydrophilic head of lipids respectively. In the bilayer structure consisting of these amphiphilic molecules, the A-blocks form a dense molecular solution inside the bilayer and the B-blocks are repelled toward the outside. This means that the average molecular density of Ablocks in this dense solution, denoted by $\left\langle\rho_{p \mathrm{~A}}^{\prime}\right\rangle_{\text {inside }}$, can be evaluated by the mean field theory of a single component dense homopolymer solution i.e. eq. (2.17). Therefore,

$$
\left\langle\rho_{p \mathrm{~A}}^{\prime}\right\rangle_{\text {inside }} \cong \frac{3 v_{\mathrm{AA}}^{\prime}}{4 w_{\mathrm{AAA}}^{\prime}}=40.4
$$

is obtained.

\subsection{Particle-Particle/Particle-Mesh (PPPM) method}

In order to use the excess free energy of non-bonded interaction in a particle-based computer simulation, the local densities, $\rho_{p \mathrm{~A}}^{\prime}$ and $\rho_{p \mathrm{~B}}^{\prime}$, have to be expressed in terms of the explicit particle coordinates. Formally, the relation is given by

$$
\rho_{p \mathrm{~A}}^{\prime}(\boldsymbol{r})=\sum_{i \mathrm{~A}} \frac{R_{e}^{3}}{N} \delta\left(\boldsymbol{r}-\boldsymbol{r}_{i \mathrm{~A}}\right),
$$


Table 2.1: Virial coefficients for solvent-free linear amphiphiles used in the present thesis.

$\begin{aligned} v_{\mathrm{AA}}^{\prime} & =5.15 \\ v_{\mathrm{BB}}^{\prime} & =0.01 \\ v_{\mathrm{AB}}^{\prime} & =-1.775 \\ w_{\mathrm{AAA}}^{\prime} & =0.095625 \\ w_{\mathrm{AAB}}^{\prime} & =0.095625 \\ w_{\mathrm{ABB}}^{\prime} & =0.095625 \\ w_{\mathrm{BBB}}^{\prime} & =0.0\end{aligned}$

where the summation runs over all A-segments in the system. The Dirac $\delta$-functions, however, are unsuitable for computations. To solve this problem, one can either regularize the $\delta$-function by a weighting function or one can use a collocation grid to compute the local densities. In the following we choose the second option, which is particularly efficient for calculating the non-bonded interactions in dense systems. The computational gain compared to the first option scales roughly with the number of particles a reference bead interacts with. Thus it is particularly suitable for systems with long-range interactions in plasma physics or astrophysics or in systems with high molecular densities (like e.g. polymer systems).

Schemes to assign particle coordinates like the PPPM method have been devised since 1980 [41]. We test and apply PPPM, as a particular example, to dense polymer and monomer systems.

\subsubsection{Original PPPM method}

We are simulating linear polymers with A-B binary component segments by beadspring model. Our coarse-grained segments are bonded by a harmonic spring potential, eq. (2.1).

In addition to this intra-molecular interaction potential, external pair interaction potential acts between the coarse-grained segments (see also section 3.2). We assume that our system is at high density; there are no solvent molecules in the system.

The external pair interaction potential, however, requires long computational times at high density owing to a large number of segments within the cutoff distance. We can overcome this problem, after dividing the system box into $M_{\text {cell }}$ spatially fixed cubic cells, by replacing the pair potential by a grid-based energy in each divided cell. This scheme is called Particle-Particle/Particle-Mesh (PPPM) [41]. To illustrate the technique, we use in the following section a nonbonded excess free energy that is suitable for dense, nearly incompressible polymer melts that are comprised of two segment species A and B. The technique is straight forwardly carried out to the compressible, solvent-free model for bilayer membranes.

We assume that our phenomenological total non-bonded interaction energy in 
the system is given by,

$$
\begin{gathered}
\frac{H_{t}}{k_{B} T}=\sum_{i=1}^{M_{x}} \sum_{j=1}^{M_{y}} \sum_{k=1}^{M_{z}} \frac{\rho \Delta V}{N}\left(\chi N \phi_{A} \phi_{B}+\frac{1}{2} \kappa N\left(\phi_{A}+\phi_{B}-\phi_{0}\right)^{2}\right) \\
\phi_{\alpha}=\frac{U_{\alpha}}{\rho \Delta V}
\end{gathered}
$$

where $(i, j, k)$ represent indices of cells, $\left(M_{x}, M_{y}, M_{z}\right)$ the number of cells in $x, y, z$ direction respectively i.e. $M_{\text {cell }}=M_{x} M_{y} M_{z}, \rho$ is the average number density of the total segments over the system box, $\Delta V$ the volume of the divided cell, $N$ polymerization degree, $U_{\alpha}$ the number of the $\alpha$-segments in the cell, and $\alpha=A, B$. Here the reference density, $\phi_{0}$, is set to unity. The Flory-Huggins parameter, $\chi N$, describes the incompatibility between the $\mathrm{A}$ and $\mathrm{B}$ species and the inverse compressibility $\kappa N$ is chosen large enough to suppress density fluctuations on the length scale, $R_{e}$.

Whereas this original method can effectively reduce the computational time, there are no non-bonded interactions between particles in neighboring (different) cells. While the segment moves within one cell, the interaction energy between this segment and the others does not change. When the segment crosses the border between the cells, impulsive interaction force suddenly acts. These situations sometimes cause simulation artifacts. Phase separation of dense simple liquids is illustrated as an example of such artifacts. In this system, neighboring cells are filled with one molecular species independently of other cells.

\subsubsection{PPPM improved with a linear interpolation}

To reduce this spatial discontinuity in the interaction, we often adopt a PPPM method improved with the linear interpolation [41-43].

Although we still use the same Hamiltonian (2.23), we change formulation about the volume fraction $\phi_{\alpha}$. In the original method, volume fraction concerning one particle, $1 / \rho \Delta V$, is added to the grid point where this particle belongs. In the improved method, with the linear interpolation, $1 / \rho \Delta V$ is divided by the 8 grid 
points which are surrounding this particle:

$$
\begin{aligned}
& \phi_{\alpha}^{\prime}(i, j, k)=\frac{\left(R_{i+1}^{x}-r_{x}\right)\left(R_{j+1}^{y}-r_{y}\right)\left(R_{k+1}^{z}-r_{z}\right)}{\Delta V} \frac{1}{\rho \Delta V} \\
& \phi_{\alpha}^{\prime}(i, j, k+1)=\frac{\left(R_{i+1}^{x}-r_{x}\right)\left(R_{j+1}^{y}-r_{y}\right)\left(r_{z}-R_{k}^{z}\right)}{\Delta V} \frac{1}{\rho \Delta V} \\
& \phi_{\alpha}^{\prime}(i, j+1, k)=\frac{\left(R_{i+1}^{x}-r_{x}\right)\left(r_{y}-R_{j}^{y}\right)\left(R_{k+1}^{z}-r_{z}\right)}{\Delta V} \frac{1}{\rho \Delta V} \\
& \ldots \\
& \phi_{\alpha}^{\prime}(i+1, j+1, k+1)=\frac{\left(r_{x}-R_{i+1}^{x}\right)\left(r_{y}-R_{j}^{y}\right)\left(r_{z}-R_{k}^{z}\right)}{\Delta V} \frac{1}{\rho \Delta V}
\end{aligned}
$$

where $(i, j, k)$ represent the indices of the grid point where the particle belongs, $\alpha$ segment species of the particle, $\phi_{\alpha}^{\prime}$ the divided volume fraction on each surrounding grid point, $\left(r_{x}, r_{y}, r_{z}\right)$ the particle coordinate, $\left(R_{v_{x}}^{x}, R_{v_{y}}^{y}, R_{\nu_{z}}^{z}\right)$ the coordinate of the grid point specified by indices $\left(v_{x}, v_{y}, v_{z}\right)$. We can obtain the total volume fraction on each grid point by summing $\phi_{\alpha}^{\prime}$ over all the particles adjacent to the grid point.

Without huge computational cost (about a factor of 2) this improved technique relieves the problems on the method mentioned in section 2.3.1.

\subsubsection{Program test}

As a program test, using the linear interpolation method, we simulate linear diblock copolymers and linear homopolymer blend in the cubic system box via canonical Monte Carlo simulations. Interactions have been defined in section 2.3.1. In all the simulations, parameters are: polymerization degree $N=32$, the total number of polymers $n_{p}=1000$, average number density of all kinds of the polymers $\rho_{0}=$ $122.0 /\left(R_{e}\right)^{3}, \kappa N=60.0$, the width of the cell $\approx R_{e} / 6.0$, where $R_{e}$ is the end to end distance of an ideal linear chain and set as a unit length. After $10^{5} \mathrm{MCS}$, during which the system relaxes, particle configuration is collected every 1000 MCS and 10 independent data are sampled. We vary parameter $\chi N$ and observe the phase behavior.

\subsection{3-a Diblock copolymers}

First, we simulate diblock copolymers. The block ratio is fixed at 0.5. Müller and Daoulas simulated this system via Single-Chain-in-Mean-Field method and calculated the free energy difference between the ordered phase and the disordered phase based on thermodynamic integration and expanded ensemble simulation techniques [36]. 
We take snapshots of the system to check the phase behavior. These snapshots show the disordered state at $\chi N \lesssim 14.0$ whereas lamellae are observed at $\chi N \gtrsim 16.0$ (Fig. 2.2). To investigate this change in phase behavior, we also calculate the density distribution of A-segments inside a lattice cell (Fig. 2.3). We can observe the sudden change in this density distribution from a single-peaked to a binodal distribution around $\chi N \approx 14.0$, which indicates the transition between the disordered phase and the lamella phase.

These results are consistent with the mean field theory that predicts the microphase separation at $\chi N=10.5[36,45]$ although its value is shifted due to the fluctuations which are ignored in the mean field theory [46].

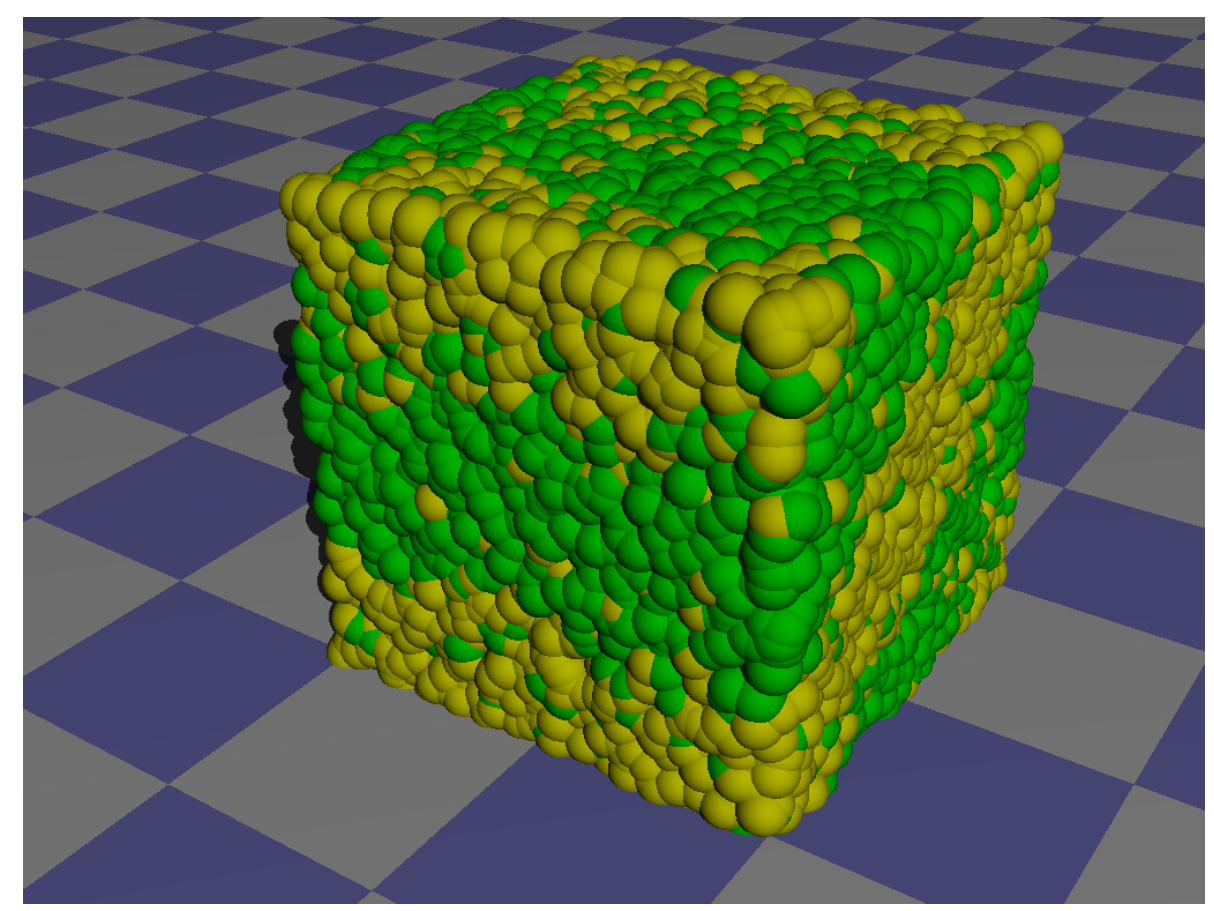

Figure 2.2: Snapshot of diblock copolymers at $\chi N=16.0$. Lamellae are observed in the system.

\subsection{3-b Homopolymer blend}

Second, we simulate the homopolymer blend, which has the same number of Ahomopolymers and B-homopolymers. We control the parameter $\chi N$ and monitor the phase behavior. Figure 2.4 shows the change in the density distribution of A-segments with the parameter $\chi N$. At $\chi N \approx 4.0$ the sudden change of this distribution is observed, which shows that macro phase separation occurs in this region.

The transition point is shifted to a larger $\chi N$ compared to the prediction $\chi N=$ 2 of the mean field approximation [45] owing to the finite size effect and, more importantly, fluctuations. 
Density profile of segment A for diblock copolymers

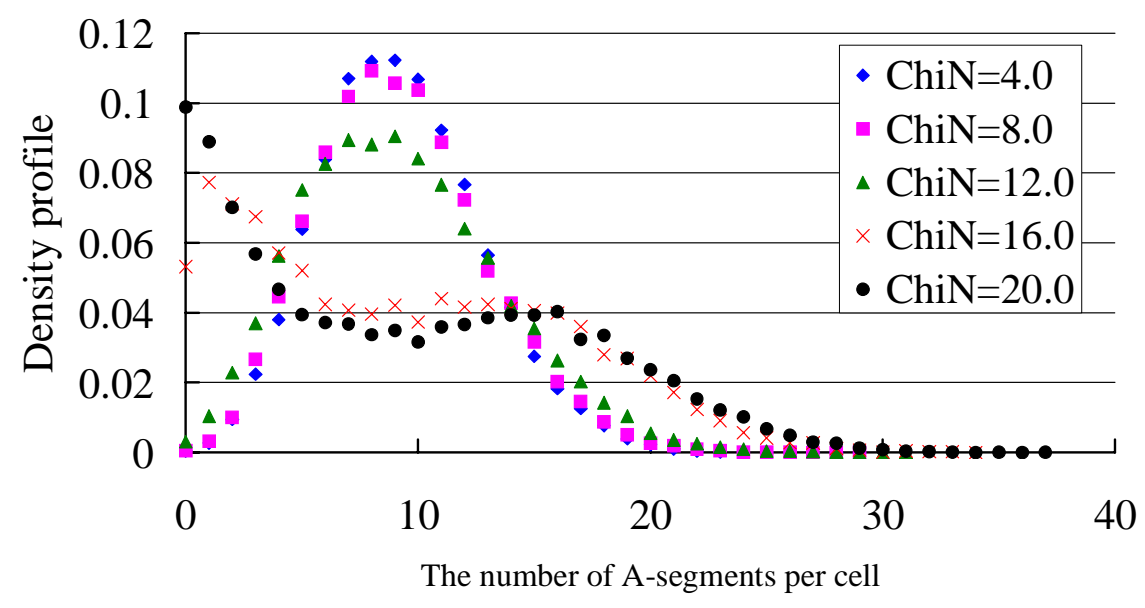

Figure 2.3: Distribution of A-segments for diblock copolymers. Micro phase separation occurs around $\chi N \approx 14.0$.

Density profile of segment A for homopolymer blends

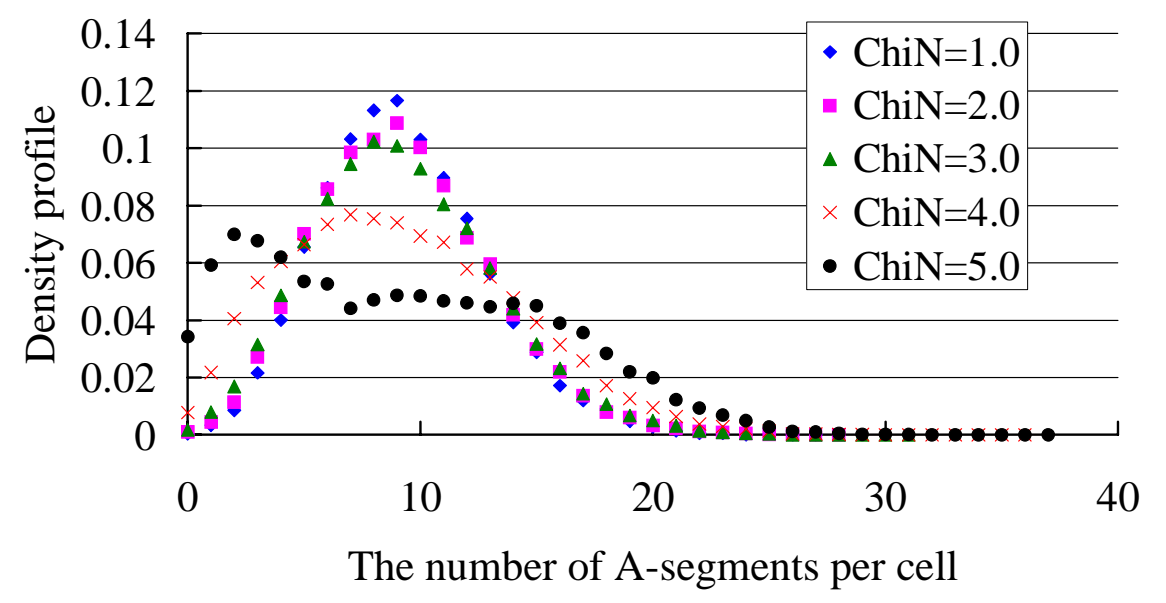

Figure 2.4: Distribution of A-segments for homopolymer blend. Macro phase separation occurs around $\chi N \approx 4.0$. 


\subsubsection{Benchmark}

We have carried out benchmark tests to compare the original method and the linear extrapolation. We simulate different systems and monitor the difference in the computational time between both the methods. The programs implemented based on these methods are compiled by $\mathrm{g}++$ version 4.0.1 with optimization option -O3. These benchmark programs are run on a server placed in our institute (Intel(R) Xeon(TM) CPU 2.40GHz, 32 bit Linux OS).

\subsection{4-a Benchmark on monomer blend}

Benchmark on 1:1 A-B monomer blend that is a mixture of $50 \%$ A-segments and $50 \%$ B-segments. The simulation parameters and benchmark results are shown in Tables 2.2 and 2.3 respectively.

Table 2.2: Simulation parameters for benchmark on 1:1 A-B monomer blend.

$\begin{aligned} \chi \chi & =5.0 \\ \kappa & =30.0 \\ \text { The total number of monomers } & =64000 \\ \text { The average number of monomers in one cell } & =64 \\ \text { The number of cells } & =1000 \\ \text { Calculated steps } & =1000 \mathrm{MCS}\end{aligned}$

Table 2.3: Computational time for 1:1 A-B monomer blend.

\begin{tabular}{rc}
\hline & Time $(\mathrm{sec})$ \\
\hline Original method & 78 \\
Improved method & 205 \\
\hline
\end{tabular}

\subsection{4-b Benchmark on diblock copolymers}

Benchmark on A-B diblock copolymers. Tables 2.4 and 2.5 show the parameter set of the simulation and the benchmark results.

Test simulations and benchmarks discussed indicate that our linear extrapolation method and implementation yield reasonable physical property and the computational cost approximately 2.5 times larger than the computational time for the original method. 
Table 2.4: Simulation parameters for benchmark on A-B diblock copolymers, where the end to end distance of an ideal chain is defined as the unit length.

\begin{aligned} & Polymerization degree $N=32 \\ & \chi N=20.0 \\ & \kappa N=50.0 \\ &$ Block ratio $=0.5 \\ &$ The number of polymers $=8000 \\ &$ Number density of polymers $=122.0 \\ &$ Width of the cell $\approx 1.0 / 6.0 \\ &$ Calculated steps $=500 \mathrm{MCS} \\ &$\hline\end{aligned}

Table 2.5: Computational time for A-B diblock copolymers.

\begin{tabular}{rc}
\hline & Time $(\mathrm{sec})$ \\
\hline Original method & 274 \\
Improved method & 642 \\
\hline
\end{tabular}




\subsection{Simulation of solvent-free homopolymers}

Using the solvent-free system discussed in section 2.1, we simulate a single component linear homopolymers in the canonical ensemble. The phase behavior observed in the simulation is compared with the results from the mean field theory in section 2.1. The bonded and non-bonded interaction potentials between the segments in our simulation system is described by equations (2.1) and (2.11), respectively.

In our simulation, we control the parameters $\rho_{p}^{\prime}$ and $v^{\prime}$ whereas the other parameters are fixed. These fixed parameters are shown in Table 2.6. $k_{B} T$ is the energy unit. The homopolymers are arranged in the rectangular parallelepiped system box with the size $x \times y \times z=L \times L \times 2 L$. Mainly the simulation results at $n_{p}=250$ homopolymers are shown in the present thesis. We have verified, however, that our simulation results do not substantially change where we simulate a larger system with $n_{p}=2000$ homopolymers.

After $3 \times 10^{6} \mathrm{MCS}$ at $\rho_{p}^{\prime} / \rho_{p c}^{\prime}=0.1,1.0,4.0$, and 10.0 and $10^{6} \mathrm{MCS}$ at other densities, by which the system relaxes to the equilibrium state, we acquire data every $10^{4}$ MCS and get 10 independent samples of the segment configurations.

Table 2.6: Simulation parameters in the single component solvent-free homopolymers.

\begin{aligned} & Polymerization degree $N=32 \\ & w^{\prime}=1.0 \\ &$ The number of polymers $n_{p}=250 \\ &$ The width of the cell $\Delta L \approx(1.0 / 6.0) R_{e} \\ &$ The maximum trial displacement $=\Delta L \\ &$\hline\end{aligned}

\subsubsection{Locating the liquid-gas coexisting line}

Prior to simulation results, we discuss two methods for locating the liquid-gas coexisting line in molecular simulation.

\subsection{1-a Methods via rectangular parallelepiped system box}

Using a rectangular parallelepiped system box, we can determine the liquid-gas coexisting line.

Our particles are arranged in a rectangular parallelepiped system box with size $x \times y \times z=L \times L \times L_{z}$, where $L<L_{z}$. We assume that the particles separate into the two coexisting phases i.e. a dense polymermelt and a dilute gas, after the simulation starts. In order to quantitatively discuss the phase transition between the gas phase and the liquid phase and construct the phase diagram, the density profile along $z$-axis is calculated.

Owing to the anisotropy of the rectangular parallelepiped system box in $z$ direction, the interfaces between both the phases tend to be parallel with $x y$-plane when the system is located on the coexisting line. We can find these interfaces from 
the density profile along $z$-axis and quantitatively understand the phase behavior. If the initial average density, $\rho_{p}^{\prime}$, is located inside the miscibility gap, the system will phase-separate into two domains. The properties in the interior of the domains correspond to those of the two coexisting phases.

This method can be applied to a variety of systems. It is well suited for strongly segregated systems deep inside the miscibility gap. Upon approaching the critical point, however, the method fails because

i) the interface that separates the two domains becomes very broad and comparable to the system size and

ii) strong fluctuation make the monitoring of the density profiles increasingly difficult.

\subsection{1-b Block density distribution technique}

In block density distribution technique [47] the system box, cubic or rectangular parallelepiped, is divided into finite small blocks or cells. Each block holds particles in response to the phase of the block. We calculate the density profile of the particles per block.

When the system is located in the homogeneous phase, i.e. the gas phase or the liquid phase, this density profile shows one sharp peak on the average density and the density of each cell is fluctuating around this peak.

Inside the miscibility gap, two peaks are found in the density profile at low density and high density; these peaks correspond to the blocks in the gas phase and the ones in the liquid phase respectively. Therefore, finding these two peaks at the same time we obtain a rough approximation of the liquid-gas coexisting line.

\subsubsection{Simulation results of solvent-free homopolymers}

For the sake of intuitively understanding the phase behavior of our system, we take the snapshots of the system. As a particular exmaple, Fig. 2.5 shows the snapshots of the system at $\rho_{p}^{\prime} / \rho_{p c}^{\prime}=10.0$. We can observe the homogeneous phase at $v^{\prime} / v_{c}^{\prime}=5.0$ though at $v^{\prime} / v_{c}^{\prime}=6.0$ the system is separated into two phases, low density and high density. At $\rho_{p}^{\prime} / \rho_{p c}^{\prime}=10.0$, in view of our snapshots, the system is in the homogeneous phase at $v^{\prime} / v_{c}^{\prime} \lesssim 5.0$ and the separated phase at $v^{\prime} / v_{c}^{\prime} \gtrsim 6.0$. In order to quantitatively discuss the phase transition between the homogeneous phase and the coexisting phase and construct an approximation of the phase diagram, the density profile along $z$-axis is calculated (see section 2.4.1a). Owing to the anisotropy of the rectangular parallelepiped system box in $z$ direction, the interface in the separated region tends to be parallel with $x y$-plane. We can find this interface from the density profile along $z$-axis and quantitatively understand the phase behavior since, in the homogeneous phase, the interface is not found and the density profile takes homogeneous form. Figure 2.6 shows this density profile along $z$-axis at $\rho_{p}^{\prime} / \rho_{p c}^{\prime}=10.0$. We can observe the phase separation 

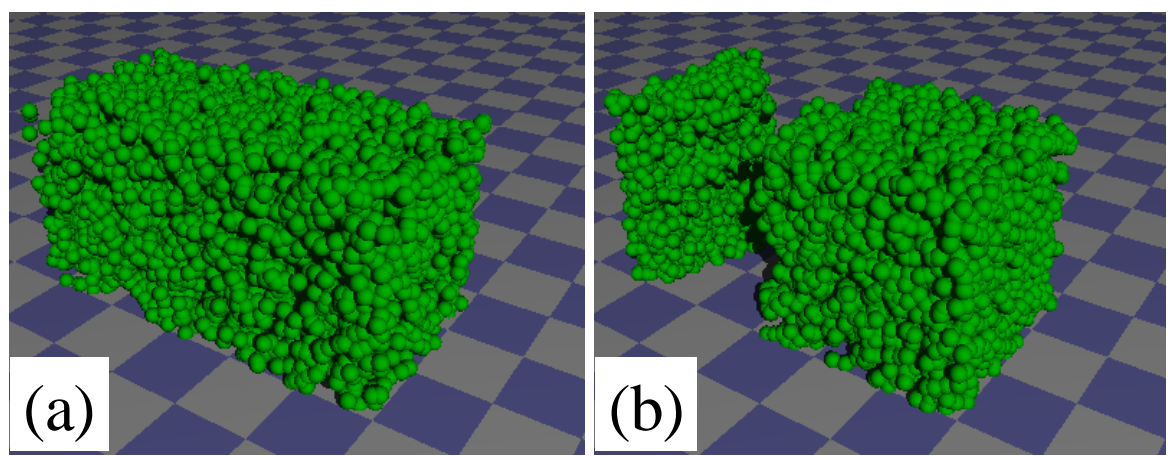

Figure 2.5: Snapshots of the system at $\rho_{p}^{\prime} / \rho_{p c}^{\prime}=10.0$ and $3 \times 10^{6}$ MCS. The snapshot (a) is taken at $v^{\prime} / v_{c}^{\prime}=5.0$ and (b) at $v^{\prime} / v_{c}^{\prime}=6.0$. These figures show the phase transition around this region.

Density profile along $z$-axis at $\rho_{\mathrm{p}}^{\prime} / \rho_{\mathrm{pc}}^{\prime}=10.0$

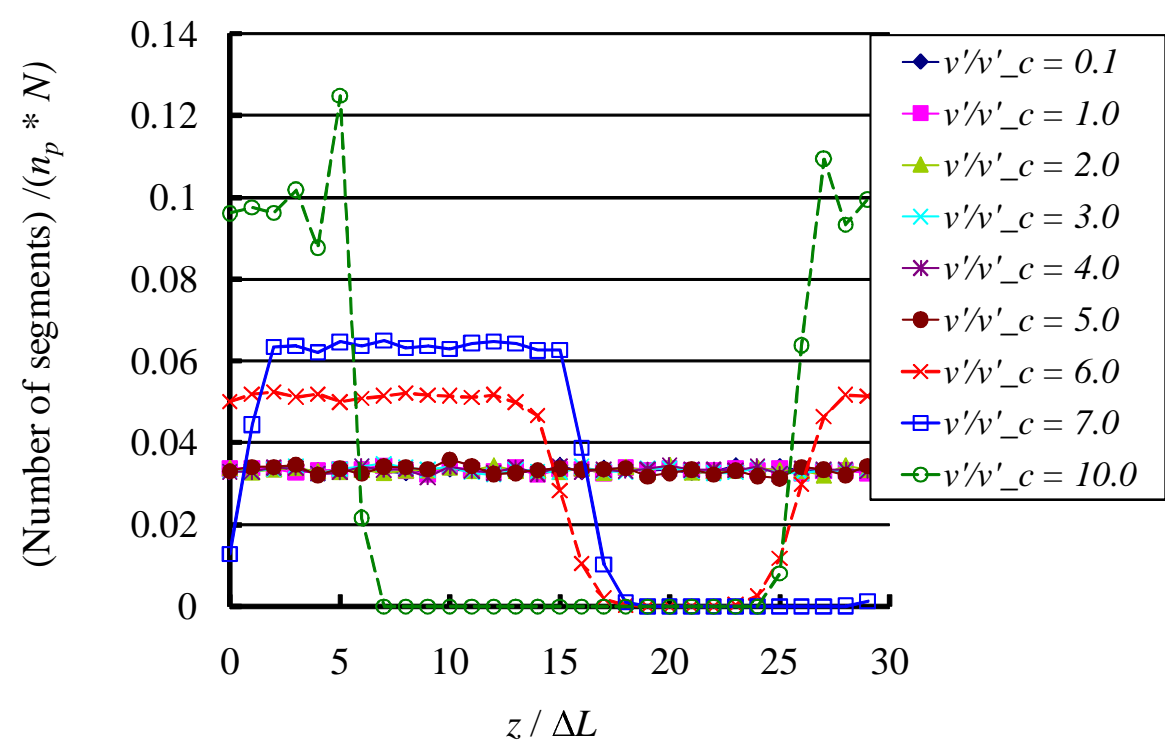

Figure 2.6: Density profile along $z$-axis at $\rho_{p}^{\prime} / \rho_{p c}^{\prime}=10.0$. Phase transition is observed at $v^{\prime} / v_{c}^{\prime} \approx 5.5 .304 L=2 L$.

at $v^{\prime} / v_{c}^{\prime} \gtrsim 6.0$, which is signaled by the formation of two domains (see Fig. 2.5). These results indicate that, with use of snapshots of the system and the density profile along $z$-axis, we can quantitatively construct the phase diagram. 
Figure 2.7 shows the binodal line of our solvent-free single component linear homopolymer systems. Though the form of the binodal line determined from numerical calculation based on the mean field theory qualitatively correspond to our simulation results, the critical point is shifted to the region $\rho_{p}^{\prime} / \rho_{p c}^{\prime} \approx 3.0$ and $v^{\prime} / v_{c}^{\prime} \approx 4.3$.

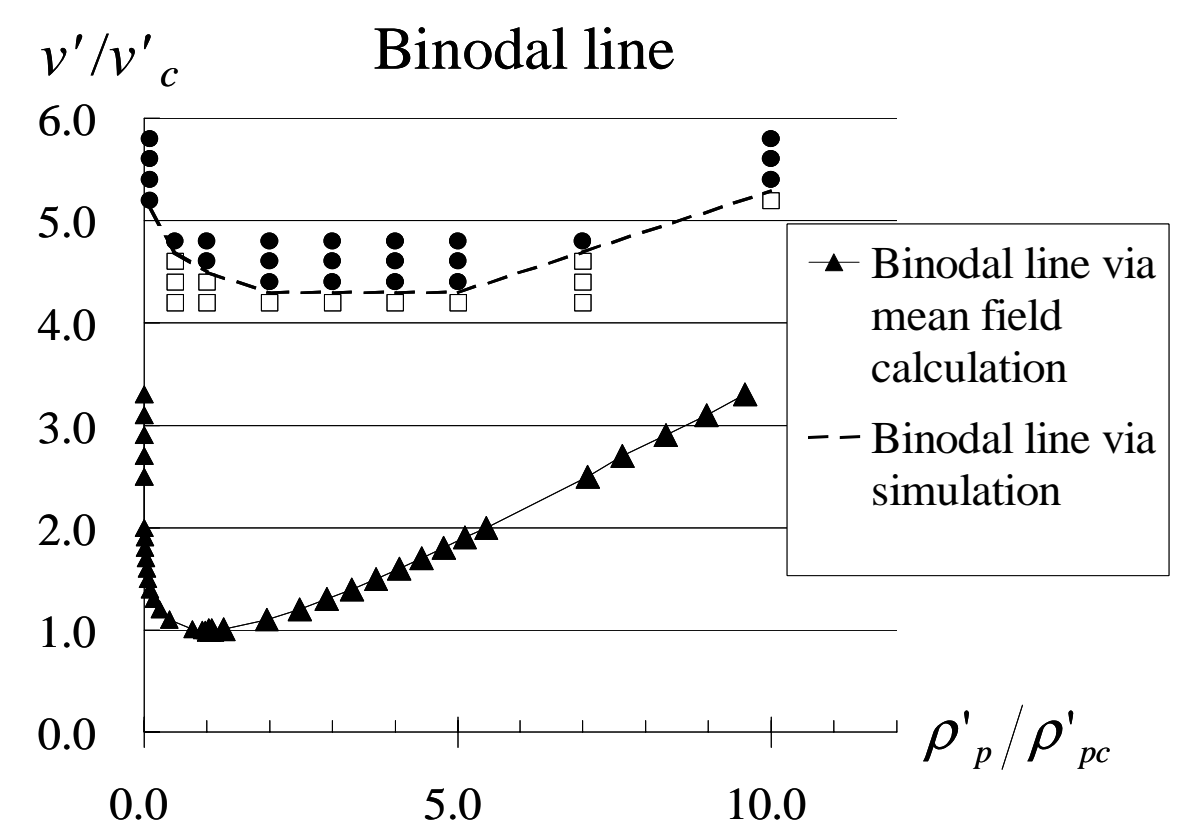

Figure 2.7: Phase diagram obtained via simulation. A black circle represents the separated phase and a white square the homogeneous phase.A binodal line is plotted by a broken line. Triangles represent the binodal line via the mean field approximation.

In order to elucidate the discrepancy between these two binodal lines, we calculate the density distribution of the segments per cell. In view of the snapshots of the system (see Fig. 2.8), the segments are locally surrounded with a void and the sparse segment configuration is observed in the rectangular parallelepiped system box. Figure 2.9 shows the density profile of the segments per cell at $\rho_{p}^{\prime} / \rho_{p c}^{\prime}=1.0$. Each cell contains a small number of segments. These results indicate that one segment interacts with only a few others. At high density, mean field approximation approaches to the exact solution whereas at low density it fails [45]. Consequently, due to the sparse segment configuration in our system, the location of the binodal line expected via the mean field approximation does not agree with our Monte Carlo simulation results. At high density, however, the binodal line predicted by the mean field theory approaches to the one from the simulation (see Fig. 2.7). At $\rho_{p}^{\prime} / \rho_{p c}^{\prime}=100$ and $w^{\prime}=1.0$ the simulation results show the phase transition at $v^{\prime} / v_{c}^{\prime} \approx 36.0$ (see Fig. 2.11), which is consistent with the binodal point located with eq. (2.17) i.e. $\rho_{p}^{\prime} / \rho_{p c}^{\prime}=100$ and $v^{\prime} / v_{c}^{\prime}=33.3$. 


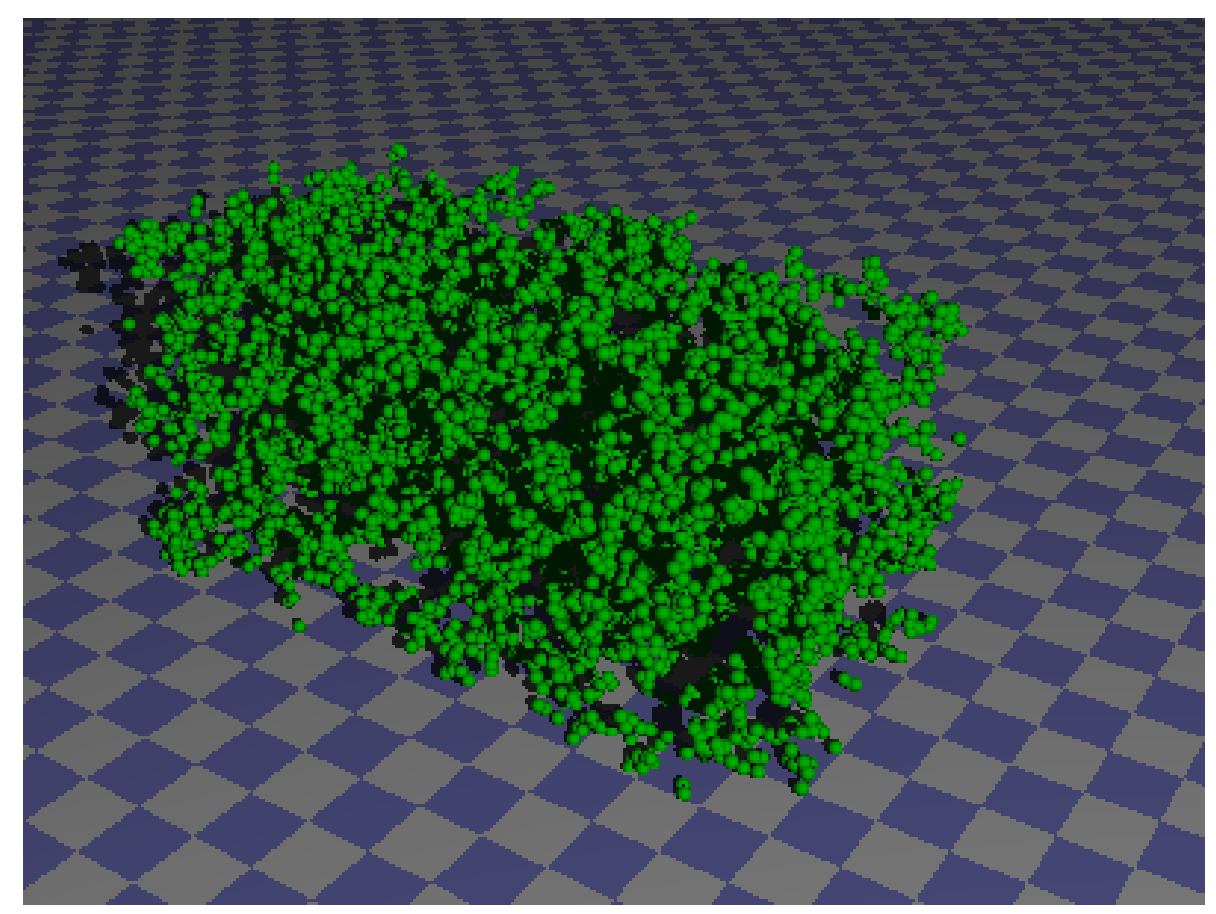

Figure 2.8: Snapshot of the system at $\rho_{p}^{\prime} / \rho_{p c}^{\prime}=1.0$ and $v^{\prime} / v_{c}^{\prime}=0.1$, taken at $3 \times 10^{6}$ MCS.

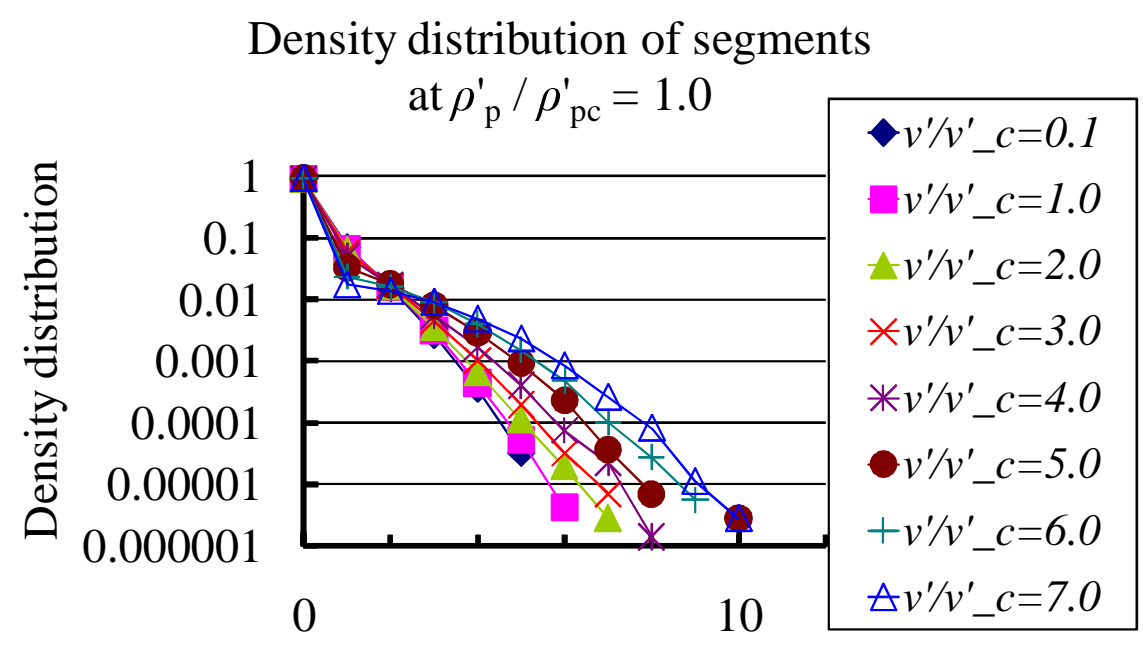

The number of segments per cell

Figure 2.9: The density profile of the segments per cell at $\rho_{p}^{\prime} / \rho_{p c}^{\prime}=1.0$. Each cell contains only a few segments. 
Next, we discuss the density distribution of the segments per cell. The density distribution at $\rho_{p}^{\prime} / \rho_{p c}^{\prime}=1.0$ (see Fig. 2.9) shows only one sharp peak in the number of the segments per cell $=0$ even though the system is in the phase-separated state (see also Fig.2.7). In the region around the number of the segments $U=2$ and high $v^{\prime} / v_{c}^{\prime}$, however, the distribution forms a shoulder. These results indicate that the peak in the high density region is covered in the sharp peak in the low density region due to the low average density. For the sake of searching for the peak in the high density region in the separated phase, we simulate the system at high average density and calculate the density distribution of the segments per cell.

Figures 2.10 and 2.11 show the density profile of the segments per cell at $\rho_{p}^{\prime} / \rho_{p c}^{\prime}=10.0$ and $\rho_{p}^{\prime} / \rho_{p c}^{\prime}=100.0$ respectively. At $\rho_{p}^{\prime} / \rho_{p c}^{\prime}=10.0$, the low peak in the high density region appears around the number of segments per cell $U=3$. On the other hand at $\rho_{p}^{\prime} / \rho_{p c}^{\prime}=100.0$, extremely high average density, the segment distribution becomes the same form as the dense polymer solutions [45]. These results correspond to our discussion in this section and the physical properties of common polymer systems. Upon increasing the incompatibility $v^{\prime} / v_{c}^{\prime}$, we observe that the distribution changes from a single-peak structure, which characterizes the homogeneous phase to a binodal form. The two peaks mark the density of the two coexisting phases. The incompatibility, at which the distribution changes its qualitative form, provides a rough estimation for the location of the binodal.

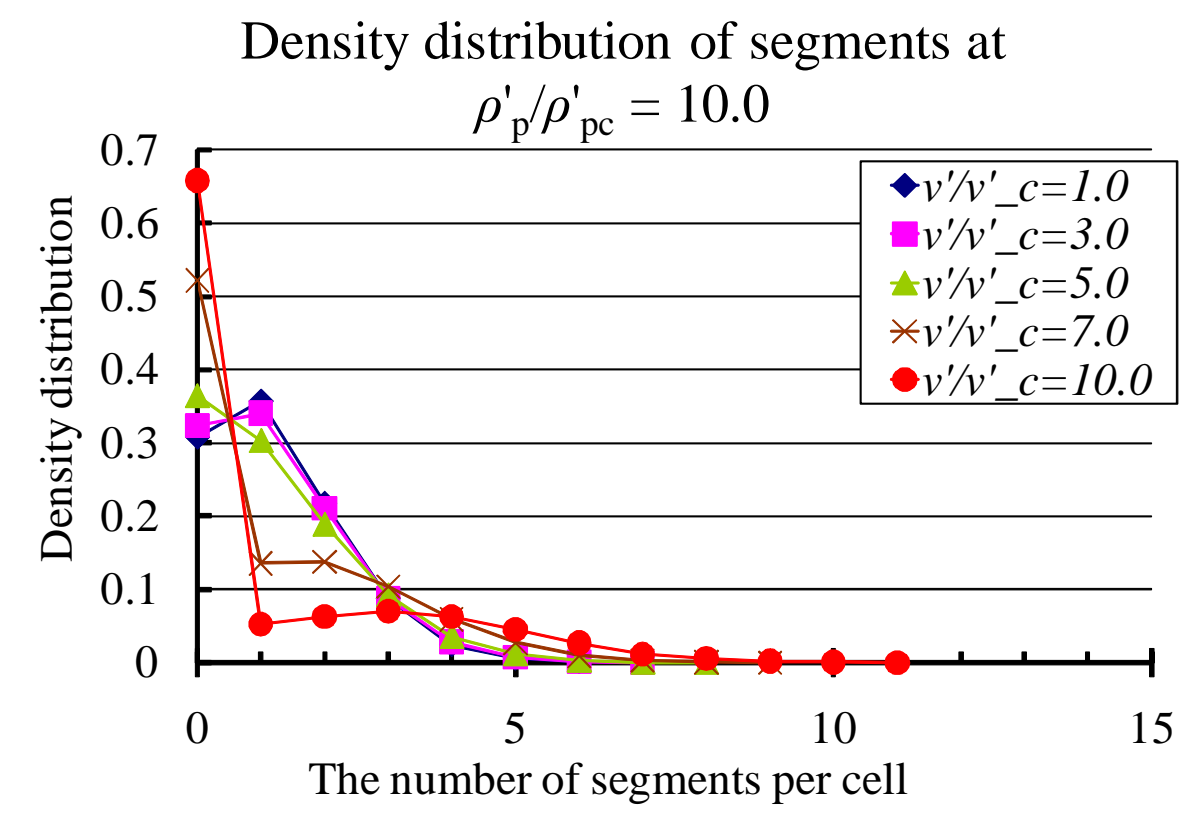

Figure 2.10: The density profile of the segments per cell. The low second peak is observed at $v^{\prime} / v_{c}^{\prime}=10.0$ around the number of segments per cell $=3$. 
Density distribution of segments

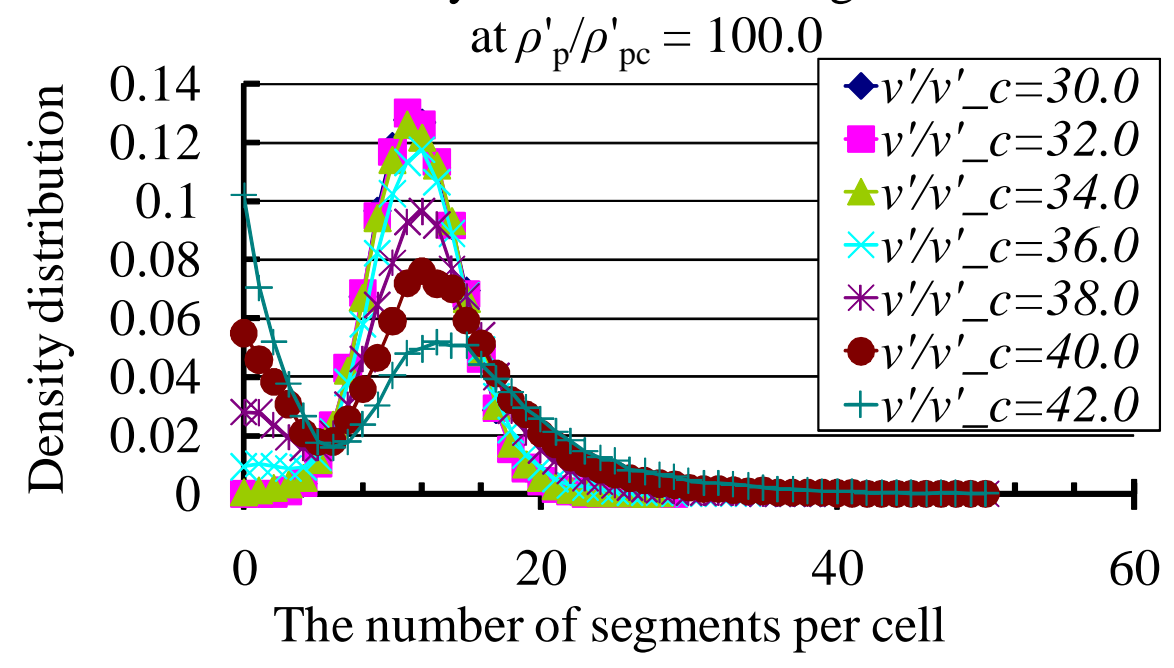

Figure 2.11: The density profile of the segments per cell at $\rho_{p}^{\prime} / \rho_{p c}^{\prime}=100.0$ and $n_{p}=2000$. The phase transition occurs around $v^{\prime} / v_{c}^{\prime} \approx 36.0$. 


\section{Chapter 3}

\section{Simulation method}

Molecular simulation, also called particle simulation, refers to computational techniques for studying phenomena observed in systems composed of a large number of particles. Two techniques can be distinguished:

One is the molecular dynamics (MD) method that is based on the fact that a set of particles move according to Newton equations. For the purpose of simulating the time evolution of systems, Newton equations are numerically solved in this method. MD is suited to study dynamical physical properties.

The other is the Monte Carlo (MC) method. In MC methods, particle space coordinates are stochastically generated according to a Boltzmann factor with use of random numbers on computers. This method allows studying equilibrium physical properties.

In the present dissertation, we choose the MC method, which is discussed in this chapter. The Mersenne Twister algorithm is adopted as a uniform random number generator in our simulation (see section 3.1.3). Our systems are simulated using periodic boundary condition, which is suitable for simulating bulk physical properties.

\subsection{NVT-Monte Carlo simulation technique}

In this section, $\mathrm{MC}$ simulation techniques in the canonical ensemble [48-50] are discussed. The canonical ensemble is also called NVT-ensemble due to the constant number of particles $N$, constant system volume $V$, and constant temperature $T$.

We assume that our system is composed of $N$ particles with mass $m$ in the canonical ensemble and that Hamiltonian of the system is defined as

$$
H\left(\boldsymbol{p}_{1}, \boldsymbol{p}_{2}, \ldots, \boldsymbol{p}_{N}, \boldsymbol{r}_{1}, \boldsymbol{r}_{2}, \ldots, \boldsymbol{r}_{N}\right)=\sum_{j=1}^{N} \frac{\boldsymbol{p}_{j}^{2}}{2 m}+U\left(\boldsymbol{r}_{1}, \ldots, \boldsymbol{r}_{N}\right),
$$

where $\boldsymbol{p}_{j}$ and $\boldsymbol{r}_{j}$ denote the momentum and the coordinates of the particle $j$ respectively and $U$ does the potential energy of the system. The probability that a micro- 
scopic state is found in a small phase space volume $d \boldsymbol{r}^{N} d \boldsymbol{p}^{N}=\prod_{j=1}^{N}\left(d \boldsymbol{r}_{j} d \boldsymbol{p}_{j}\right)$ at the position $\left(\boldsymbol{p}_{1}, \ldots, \boldsymbol{p}_{N}, \boldsymbol{r}_{1}, \ldots, \boldsymbol{r}_{N}\right)$ is

$$
\frac{1}{Z_{N}(T, V) N ! h^{3 N}} \exp \left[-\frac{1}{k_{B} T} H\left(\boldsymbol{p}_{1}, \ldots, \boldsymbol{p}_{N}, \boldsymbol{r}_{1}, \ldots, \boldsymbol{r}_{N}\right)\right] d \boldsymbol{r}^{N} d \boldsymbol{p}^{N}
$$

where $h$ denotes Planck's constant and $Z_{N}(T, V)$ does the partition function of the system,

$$
Z_{N}(T, V)=\frac{1}{\Lambda^{3 N} N !} \int d \boldsymbol{r}_{1} \ldots d \boldsymbol{r}_{N} \exp \left[-\frac{1}{k_{B} T} U\left(\boldsymbol{r}_{1}, \ldots, \boldsymbol{r}_{N}\right)\right], \quad \Lambda:=\frac{h}{\sqrt{2 \pi m k_{B} T}} .
$$

The ensemble average of a mechanical quantity, $A\left(\boldsymbol{r}_{1}, \boldsymbol{r}_{2}, \ldots, \boldsymbol{r}_{N}\right)$, which depends only on the particle coordinates is given by

$$
\begin{aligned}
\langle A\rangle_{N V T}= & \frac{1}{Z_{N}(T, V) N ! h^{3 N}} \\
& \times \iint\left\{A\left(\boldsymbol{r}_{1}, \ldots, \boldsymbol{r}_{N}\right) \exp \left[-\frac{1}{k_{B} T} H\left(\boldsymbol{p}_{1}, \ldots, \boldsymbol{r}_{N}\right)\right]\right\} d \boldsymbol{r}^{N} d \boldsymbol{p}^{N} \\
= & \frac{1}{Q_{N}(T, V)} \int_{V}\left\{A\left(\boldsymbol{r}_{1}, \ldots, \boldsymbol{r}_{N}\right) \exp \left[-\frac{1}{k_{B} T} U\left(\boldsymbol{r}_{1}, \ldots, \boldsymbol{r}_{N}\right)\right]\right\} d \boldsymbol{r}_{1} \ldots d \boldsymbol{r}_{N}
\end{aligned}
$$

where

$$
Q_{N}(T, V)=\int_{V} d \boldsymbol{r}_{1} \ldots d \boldsymbol{r}_{N} \exp \left[-\frac{1}{k_{B} T} U\left(\boldsymbol{r}_{1}, \ldots, \boldsymbol{r}_{N}\right)\right] .
$$

The right-hand side of (3.2) involves a $N$-dimensional integral over the whole system volume $V$. At $N \gg 1$, high dimension prevents us from numerically calculating the integral via deterministic integration, e.g. trapezium rule and Simpson's rule. For such a high dimensional integral, Monte Carlo integration is required to determine the ensemble average $\langle A\rangle_{N V T}$, i.e. a physical property of our system.

\subsubsection{Metropolis algorithm}

In NVT-Monte Carlo simulation, a series of particle coordinates in canonical ensemble are sampled in a Markov process. The particle configurations correspond to the states in the Markov process. In 3-dimensional systems, each configuration is a set of $3 N$ microscopic particle coordinates, $\left(\boldsymbol{r}_{1}, \ldots, \boldsymbol{r}_{N}\right)$. The set of all the states is denoted by $\Omega=\{1,2,3, \ldots, k, \ldots\}$. Within the MC simulation, a stochastic trajectory through configuration space is constructed, such that the distribution of visited configurations obeys the canonical distribution according to the partition function eq. (3.1). The probability density that one random walker is found on a state $i$ is:

$$
w_{i}:=\frac{1}{Z_{N}(T, V)} \frac{1}{\Lambda^{3 N} N !} \exp \left[-\frac{1}{k_{B} T} U_{i}\right]
$$


where $U_{i}$ denotes the potential energy at $i$. The transition probability changing the state from $i$ to $k$ is denoted by $P_{i k}$.

In our simulation, this transition probability and eq. (3.3) should fulfill the principle of detailed balance

$$
w_{i} P_{i k}=w_{k} P_{k i}, \quad i, k \in \Omega .
$$

This principle means the frequency that the transition from $i$ to $k$ occurs is equal to the frequency that from $k$ to $i$ : while a large number of the space trajectories move over the phase space, the number of them on each point in the phase space should be constants in equilibrium. In other words, the net flow between any pairs of the points should vanish. This condition is reduced to

$$
\frac{P_{i k}}{P_{k i}}=\frac{\exp \left[-U_{k} / k_{B} T\right]}{\exp \left[-U_{i} / k_{B} T\right]}=\exp \left[-\frac{1}{k_{B} T}\left(U_{k}-U_{i}\right)\right] \text {. }
$$

In Metropolis algorithm, particle coordinates are sampled according to the equation which is obtained from (3.4),

$$
\begin{aligned}
P_{i k}: P_{k i} & =1: \exp \left[-\left(1 / k_{B} T\right)\left(U_{i}-U_{k}\right)\right], \quad \text { where } U_{k}<U_{i}, \\
& =\exp \left[-\left(1 / k_{B} T\right)\left(U_{k}-U_{i}\right)\right]: 1, \quad \text { where } U_{k}>U_{i} .
\end{aligned}
$$

If $U_{k}<U_{i}$, the system is moved from $i$ to $k$ with probability $=1$. If $U_{k}>U_{i}$, the system is moved from $i$ to $k$ with probability $=\exp \left[-\left(1 / k_{B} T\right)\left(U_{k}-U_{i}\right)\right](<1)$. In the remainder of this section 3.1.1, we discuss the detail algorithm.

1) Choosing one particle from $N$ ones at random. Giving this chosen particle $\alpha$ a random displacement $\Delta \boldsymbol{r}_{\alpha}=\left(\Delta x_{\alpha}, \Delta y_{\alpha}, \Delta z_{\alpha}\right)$. The selected particle is moved from $\boldsymbol{r}_{\alpha}$ to $\boldsymbol{r}_{\alpha}+\Delta \boldsymbol{r}_{\alpha}$. The random displacement vector $\Delta \boldsymbol{r}_{\alpha}$ is determined with three random numbers $\xi_{x}, \xi_{y}$, and $\xi_{z}$ uniformly distributed in the interval $[0,1)$.

$$
\Delta x_{\alpha}=\delta\left(1-2 \xi_{x}\right), \quad \Delta y_{\alpha}=\delta\left(1-2 \xi_{y}\right), \quad \Delta z_{\alpha}=\delta\left(1-2 \xi_{z}\right)
$$

With this displacement, points in the cubic box whose centre is located on the position $\boldsymbol{r}_{\alpha}$ with size $2 \delta \times 2 \delta \times 2 \delta$ is uniformly chosen. We call this movement the trial displacement.

2) Calculating the potential energy of the system after the trial displacement, $U_{\text {trial }}$.

3) Comparing $U_{\text {trial }}$ to the potential before the trial displacement, $U_{\text {current }}$.

(a) If $U_{\text {trial }} \leq U_{\text {current }}$, accept the state after the trial displacement and update the coordinates of the particle $\alpha$.

(b) If $U_{\text {trial }}>U_{\text {current }}$, generate one more random number, $\eta$, uniformly distributed in the interval $[0,1)$, and if

$$
\eta<\exp \left[-\left(1 / k_{B} T\right)\left(U_{\text {trial }}-U_{\text {current }}\right)\right],
$$

accept the state after the trial displacement and update the coordinates of the particle $\alpha$. Otherwise, reject the state after the trial displacement and keep the current state to the next simulation step. 
4) Return to the first procedure, 1).

This procedure 1) - 4) generates, in sequence, a series of the particle coordinates in canonical ensemble and one cycle is called one simulation step.

\subsubsection{Monte Carlo step (MCS) and acceptance ratio}

The ratio of the number of accepted simulation steps in one MCS to $N$ cycles of the Metropolis algorithm is defined as acceptance ratio. At high maximum trial displacement $\delta$, the particles can step with large strides through configuration space though the acceptance ratio decreases owing to the large energy change after the trial displacement. On the other hand, at low $\delta$ the acceptance ratio increases due to the small energy change though the particles walk at a small pace. Thus, the acceptance ratio and the relaxation time to equilibration of the system depend on the parameter $\delta$. The optimal value of the acceptance ratio for the short relaxation time is empirically about $50 \%$ [48].

\subsubsection{Mersenne Twister}

In our Monte Carlo simulation, Mersenne Twister is adopted as a uniform random number generator [51-53].

- A high order of dimensional equidistribution. This means that the correlation between successive values in the output random number sequence is negligible.

- Architected to have a period of $2^{19937}-1$. Practically, there is little demand for larger periods, since most simulations do not demand $2^{19937}-1$ unique combinations.

- The source code is available on

http://www . math.sci.hiroshima-u.ac.jp/ m-mat/MT/emt.html

This site is also found on the net with the search word "Mersenne Twister". 


\subsection{Statistics for molecules with intra-structure}

\subsubsection{Molecules with the internal structure}

We discuss the statistics about $N$ molecules of the same species in $N V T$-ensemble. One molecule consists of $M$ atoms; the total number of the atoms in the system box $=M N$. The partition function for this system is denoted by $Z_{N}(T, V)$ :

$$
\begin{aligned}
& Z_{N}(T, V)= \\
& \frac{1}{\Lambda^{3 N M} N !} \int d \boldsymbol{r}_{1}^{1} d \boldsymbol{r}_{2}^{1} \ldots d \boldsymbol{r}_{M}^{1} d \boldsymbol{r}_{1}^{2} \ldots d \boldsymbol{r}_{M}^{N} \exp \left[-\frac{1}{k_{B} T} U\left(\boldsymbol{r}_{1}^{1}, \boldsymbol{r}_{2}^{1}, \ldots, \boldsymbol{r}_{M}^{1}, \boldsymbol{r}_{1}^{2}, \ldots, \boldsymbol{r}_{M}^{N}\right)\right]
\end{aligned}
$$

where $\boldsymbol{r}_{k}^{l}$ represents the position of the $k$-th atom in the $l$-th molecule, $U$ is the potential energy in the system, and the thermal de Broglie wave length $\Lambda$ has been introduced in eq. (3.1). The potential energy $U$ is divided into 2 parts:

i) The internal potential energy, $U^{\text {int }}$, which includes the intra-molecular interactions, e.g. bonding, bond bending, and torsion.

ii) The non-bonded potential energy, $U^{\text {nb }}$, which takes into account the nonbonded interactions which have not been taken into account in the internal part.

With the use of $U^{\mathrm{int}}$ and $U^{\mathrm{nb}}$, the total potential $U$ and the partition function (3.6) are:

$$
U\left(\boldsymbol{r}_{1}^{1}, \ldots, \boldsymbol{r}_{M}^{N}\right)=U^{\mathrm{nb}}\left(\boldsymbol{r}_{1}^{1}, \ldots, \boldsymbol{r}_{M}^{N}\right)+\sum_{i=1}^{N} U^{\mathrm{int}}\left(\boldsymbol{r}_{1}^{i}, \ldots, \boldsymbol{r}_{M}^{i}\right)
$$

and

$$
\begin{aligned}
& Z_{N}(T, V)= \\
& \frac{1}{\Lambda^{3 N M} N !} \int d \boldsymbol{r}_{1}^{1} \ldots d \boldsymbol{r}_{M}^{N} \exp \left[-\frac{1}{k_{B} T}\left\{U^{\mathrm{nb}}\left(\boldsymbol{r}_{1}^{1}, \ldots, \boldsymbol{r}_{M}^{N}\right)+\sum_{i=1}^{N} U^{\mathrm{int}}\left(\boldsymbol{r}_{1}^{i}, \ldots, \boldsymbol{r}_{M}^{i}\right)\right\}\right]
\end{aligned}
$$

\subsubsection{Ideal molecules}

We define ideal molecules as molecules interacting only via the bonded potential, i.e. $U^{\mathrm{nb}}=0$. The partition function (3.8) of such an ideal molecular system is 
denoted by $Z_{N}^{\text {ideal }}(T, V)$,

$$
\begin{aligned}
Z_{N}^{\text {ideal }}(T, V) & =\frac{1}{\Lambda^{3 N M} N !} \int d \boldsymbol{r}_{1}^{1} \ldots d \boldsymbol{r}_{M}^{N} \exp \left[-\frac{1}{k_{B} T} \sum_{i=1}^{N} U^{\text {int }}\left(\boldsymbol{r}_{1}^{i}, \ldots, \boldsymbol{r}_{M}^{i}\right)\right] \\
& =\frac{1}{\Lambda^{3 N M} N !} \prod_{i=1}^{N}\left\{\int d \boldsymbol{r}_{1}^{i} \ldots d \boldsymbol{r}_{M}^{i} \exp \left[-\frac{1}{k_{B} T} U^{\mathrm{int}}\left(\boldsymbol{r}_{1}^{i}, \ldots, \boldsymbol{r}_{M}^{i}\right)\right]\right\} \\
& =\frac{1}{N !}(f(T, V))^{N} \\
& =\frac{V^{N}}{N ! \Lambda^{3 N}}(j(T, V))^{N}
\end{aligned}
$$

where

$$
f(T, V):=Z_{1}^{\text {ideal }}(T, V)=\frac{1}{\Lambda^{3 M}} \int d \boldsymbol{r}_{1} \ldots d \boldsymbol{r}_{M} \exp \left[-\frac{1}{k_{B} T} U^{\text {int }}\left(\boldsymbol{r}_{1}, \ldots, \boldsymbol{r}_{M}\right)\right]
$$

and

$$
\begin{aligned}
j(T, V) & :=\frac{\Lambda^{3}}{V} Z_{1}^{\text {ideal }}(T, V) \\
& =\frac{1}{V \Lambda^{3(M-1)}} \int d \boldsymbol{r}_{1} \ldots d \boldsymbol{r}_{M} \exp \left[-\frac{1}{k_{B} T} U^{\text {int }}\left(\boldsymbol{r}_{1}, \ldots, \boldsymbol{r}_{M}\right)\right],
\end{aligned}
$$

$\boldsymbol{r}_{k}$ is the position of the $k$-th atom in one molecule. $f(T, V)$ and $j(T, V)$ correspond to the partition function of one ideal molecule and the partition function of the internal degrees of freedom for one ideal molecule respectively.

Using Stirling's approximation,

$$
\log v \cong v \log v-v \quad \text { for an integer } v \gg 1,
$$

we can calculate the system free energy $F_{\text {ideal }}(T, V, N)$ and the system chemical potential $\mu_{\text {ideal }}(T, V, N)$ from the partition function (3.10).

$$
\begin{gathered}
\frac{F_{\text {ideal }}(T, V, N)}{k_{B} T}=-\log Z_{N}^{\text {ideal }}(T, V) \cong-N \log \left(\frac{V e}{N \Lambda^{3}} j(T, V)\right) \\
\frac{\mu_{\text {ideal }}(T, V, N)}{k_{B} T}=-\frac{\partial}{\partial N} \log Z_{N}^{\text {ideal }}(T, V) \cong \log \left(\Lambda^{3} \rho\right)-\log j(T, V)
\end{gathered}
$$

where the average molecular density in the system is denoted by $\rho=N / V$. Due to the intensive property of the chemical potential, $j(T, V)$ should not depend on $V$,

$$
j(T, V)=j(T) .
$$

With this expression for $j(T)$, we can rewrite equations (3.10), (3.13), and (3.14) in the form:

$$
Z_{N}^{\text {ideal }}(T, V)=\frac{V^{N}}{N ! \Lambda^{3 N}}(j(T))^{N},
$$




$$
\begin{gathered}
\frac{F_{\text {ideal }}(T, V, N)}{k_{B} T}=-N \log \left(\frac{V e}{N \Lambda^{3}} j(T)\right), \\
\frac{\mu_{\text {ideal }}(T, \rho)}{k_{B} T}=\log \left(\Lambda^{3} \rho\right)-\log j(T),
\end{gathered}
$$

where

$$
j(T)=\frac{\Lambda^{3}}{V} Z_{1}^{\text {ideal }}(T, V)=\frac{1}{V \Lambda^{3(M-1)}} \int d \boldsymbol{r}_{1} \ldots d \boldsymbol{r}_{M} \exp \left[-\frac{1}{k_{B} T} U^{\text {int }}\left(\boldsymbol{r}_{1}, \ldots, \boldsymbol{r}_{M}\right)\right] .
$$

\subsection{2-a Internal potential energy via the bond vector}

The internal potential energy of both an ideal molecule and a non-ideal molecule is independent of the molecular center of mass; $U^{\text {int }}\left(\boldsymbol{r}_{1}, \ldots, \boldsymbol{r}_{M}\right)$ depends only on the molecular configuration, i.e. the relative coordinates between the atoms.

The bond vector,

$$
\boldsymbol{u}_{i}:=\boldsymbol{r}_{i+1}-\boldsymbol{r}_{i}, \quad 1 \leq i \leq M-1,
$$

determines this configuration (see Fig. 3.1). Therefore, the internal potential energy is represented by the bond vector:

$$
\tilde{U}^{\text {int }}\left(\boldsymbol{u}_{1}, \boldsymbol{u}_{2}, \ldots, \boldsymbol{u}_{M-1}\right):=U^{\text {int }}\left(\boldsymbol{r}_{1}, \ldots, \boldsymbol{r}_{M}\right),
$$

where

$$
\boldsymbol{r}_{i}=\boldsymbol{r}_{1}+\sum_{j=1}^{i-1} \boldsymbol{u}_{j}, \quad 2 \leq i \leq M .
$$

With the use of the bond vector and $\tilde{U}^{\text {int }}\left(\boldsymbol{u}_{1}, \ldots, \boldsymbol{u}_{M-1}\right)$, the partition function of an ideal molecule, $Z_{1}^{\text {ideal }}(T, V)$, is

$$
\begin{aligned}
Z_{1}^{\text {ideal }}(T, V) & =\frac{1}{\Lambda^{3 M}} \int d \boldsymbol{u}_{1} \ldots d \boldsymbol{u}_{M-1} d \boldsymbol{r}_{1} \exp \left[-\frac{1}{k_{B} T} U^{\text {int }}\left(\boldsymbol{r}_{1}, \ldots, \boldsymbol{r}_{M}\right)\right] \\
& =\frac{V}{\Lambda^{3 M}} \int d \boldsymbol{u}_{1} \ldots d \boldsymbol{u}_{M-1} \exp \left[-\frac{1}{k_{B} T} \tilde{U}^{\text {int }}\left(\boldsymbol{u}_{1}, \ldots, \boldsymbol{u}_{M-1}\right)\right]
\end{aligned}
$$

\subsection{2-b Bead-spring model}

In a bead-spring model (see also section A.2) of a linear polymer,

$$
U^{\mathrm{int}}\left(\boldsymbol{r}_{1}, \ldots, \boldsymbol{r}_{M}\right)=\frac{3 k_{B} T}{2 b^{2}} \sum_{i=1}^{M-1}\left|\boldsymbol{r}_{i+1}-\boldsymbol{r}_{i}\right|^{2}=\frac{3 k_{B} T}{2 b^{2}} \sum_{i=1}^{M-1}\left|\boldsymbol{u}_{i}\right|^{2},
$$




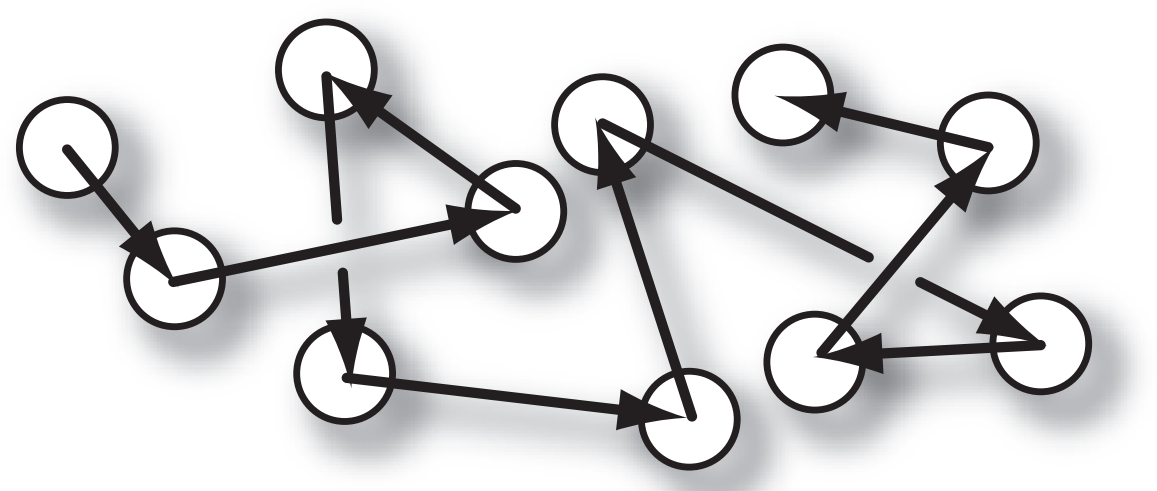

Figure 3.1: An example of a molecular configuration schematically represented by $\boldsymbol{u}_{i}$. Spheres represent the atoms. Arrows show these bond vectors, i.e. relative coordinates between the atoms, which determine the internal potential energy independent of the absolute coordinates.

where $b$ denotes the segment size. In this model,

$$
\begin{aligned}
& \int d \boldsymbol{r}_{1} \ldots d \boldsymbol{r}_{M} \exp \left[-\frac{1}{k_{B} T} U^{\mathrm{int}}\left(\boldsymbol{r}_{1}, \ldots, \boldsymbol{r}_{M}\right)\right] \\
= & \int d \boldsymbol{u}_{1} \ldots d \boldsymbol{u}_{M-1} d \boldsymbol{r}_{1} \prod_{i=1}^{M-1} \exp \left[-\frac{3}{2 b^{2}}\left|\boldsymbol{u}_{i}\right|^{2}\right]=V\left\{\int d \boldsymbol{u} \exp \left[-\frac{3}{2 b^{2}}|\boldsymbol{u}|^{2}\right]\right\}^{M-1},
\end{aligned}
$$

where $\boldsymbol{u}$ is one bond vector. At $b \ll V^{1 / 3}$, i.e. the condition that the system is far larger than the segment, this Gaussian integral over the system box is approximated by the Gaussian integral over an infinite volume:

$$
V\left\{\int d \boldsymbol{u} \exp \left[-\frac{3}{2 b^{2}}|\boldsymbol{u}|^{2}\right]\right\}^{M-1} \cong V\left(\frac{2 \pi b^{2}}{3}\right)^{\frac{3}{2}(M-1)} .
$$

Using this result, we determine $j(T)$ of the bead-spring model, (3.18).

$$
Z_{0}:=j(T)=\frac{1}{\Lambda^{3(M-1)}}\left(\frac{2 \pi b^{2}}{3}\right)^{\frac{3}{2}(M-1)}
$$

$Z_{0}$ is not explicitly dependent on $T$, but it depends on $T$ via thermal de Broglie wave length $\Lambda$ and the segment size $b$. The free energy (3.16) and the chemical potential (3.17) of the bead-spring model are also calculated:

$$
\begin{gathered}
\frac{F_{\text {ideal }}(T, V, N)}{k_{B} T}=-N \log \left(\frac{V e}{N \Lambda^{3}} Z_{0}\right) \\
\frac{\mu_{\text {ideal }}(T, \rho)}{k_{B} T}=\log \left(\Lambda^{3} \rho\right)-\log Z_{0} .
\end{gathered}
$$


In our simulation based on the bead-spring model, the segment size is fixed at $b^{2}=R_{e}^{2} /(M-1)$. Using this condition, we show $Z_{0}$ defined in (3.21) in our simulation model (see eq. (2.1)).

$$
Z_{0}=\frac{1}{\Lambda^{3(M-1)}}\left(\frac{2 \pi}{3(M-1)} R_{e}^{2}\right)^{\frac{3}{2}(M-1)}
$$

\subsubsection{Statistics of molecules in $\mu V T$-ensemble}

Based on the statistics in the canonical ensemble, we discuss the statistics in the grand canonical ensemble, i.e. $\mu V T$-ensemble.

We have a system in $N V T$-ensemble, $\left(T, V_{0}, N_{0}\right)$, where $V_{0}$ denotes the total system volume and $N_{0}$ the total number of molecules. We assume that all the molecules are identical and that each molecule is composed of $M$ atoms: the total number of the atoms in the system is $M N_{0}$. Our system is divided into 2 subsystems;

i) $\left(T, V_{0}-V, N_{0}-N\right)$. The molecules in this subsystem do not interact, i.e. ideal molecular system.

ii) $(T, V, N)$. This subsystem contains $N$ interacting molecules.

When these two subsystems are isolated from each other, the total partition function of the system is written with relations (3.15) and (3.8):

$$
\begin{aligned}
Z^{\text {iso }}\left(T, V, V_{0}, N, N_{0}\right) & =Z_{N_{0}-N}^{\text {ideal }}\left(T, V_{0}-V\right) Z_{N}(T, V) \\
& =\frac{\left(V_{0}-V\right)^{N_{0}-N}(j(T))^{N_{0}-N}}{\left(N_{0}-N\right) ! \Lambda^{3\left(N_{0}-N\right)}} Z_{N}(T, V)
\end{aligned}
$$

When our subsystems are allowed to exchange the molecules, the total partition function of the system, $Z\left(T, V, V^{\prime}, N_{0}\right)$ where $V^{\prime}:=V_{0}-V$, is determined via

$$
\begin{aligned}
Z\left(T, V, V^{\prime}, N_{0}\right) & =\sum_{N=0}^{N_{0}} Z^{\text {iso }}\left(T, V, V_{0}, N, N_{0}\right) \\
& =\sum_{N=0}^{N_{0}}\left\{\frac{V^{N_{0}-N}(j(T))^{N_{0}-N}}{\left(N_{0}-N\right) ! \Lambda^{3\left(N_{0}-N\right)}} Z_{N}(T, V)\right\}
\end{aligned}
$$

Consider this partition function in the limit that the subsystem $\left(T, V^{\prime}, N_{0}-N\right)$, composed of ideal molecules, is far larger than the subsystem $(T, V, N)$, interacting molecules; $N_{0} \rightarrow \infty, V^{\prime} \rightarrow \infty,\left(N_{0} / N\right) \rightarrow \infty$, and $\left(N_{0} / V^{\prime}\right) \rightarrow \rho_{\text {res }}$ i.e. it acts as a reservoir. This ideal molecular density $\rho_{\text {res }}$ is related to the chemical potential of the ideal molecules $\mu_{\text {ideal }}\left(T, \rho_{\text {res }}\right),(3.17)$. Using these relations, this total partition 
function of the system in this limit,

$$
\begin{aligned}
Z\left(T, V, V^{\prime}, N_{0}\right) \cong \lim _{\substack{N_{0}, V^{\prime} \rightarrow \infty \\
N_{0} / V^{\prime}=\rho_{\text {res }}}} \sum_{N=0}^{N_{0}}\left\{\frac{V^{N_{0}}(j(T))^{N_{0}}}{N_{0} ! \Lambda^{3 N_{0}}} \Lambda^{3 N}\left(\frac{N_{0}}{V^{\prime}}\right)^{N}(j(T))^{-N} Z_{N}(T, V)\right\} \\
=\lim _{\substack{N_{0}, V^{\prime} \rightarrow \infty \\
N_{0} / V^{\prime}=\rho_{\text {res }}}}\left[Z_{N_{0}}^{\text {ideal }}\left(T, V^{\prime}\right) \sum_{N=0}^{N_{0}}\left\{\exp \left[\frac{\mu_{\text {ideal }}\left(T, \rho_{\text {res }}\right)}{k_{B} T} N\right] Z_{N}(T, V)\right\}\right] .
\end{aligned}
$$

Therefore, the partition function for the interacting molecules in this limit is:

$$
\begin{aligned}
\Xi(\mu, T, V) & :=\sum_{N=0}^{\infty}\left\{\exp \left[\frac{\mu}{k_{B} T} N\right] Z_{N}(T, V)\right\} \\
& =\sum_{N=0}^{\infty}\left\{\frac{\exp \left[\left(\mu / k_{B} T\right) N\right]}{\Lambda^{3 N M} N !} \int d \boldsymbol{r}_{1}^{1} \ldots d \boldsymbol{r}_{M}^{N} \exp \left[-\frac{1}{k_{B} T} U\left(\boldsymbol{r}_{1}^{1}, \ldots, \boldsymbol{r}_{M}^{N}\right)\right]\right\}
\end{aligned}
$$

where $\mu:=\mu_{\text {ideal }}\left(T, \rho_{\text {res }}\right) . \Xi(\mu, T, V)$ is called grand partition function, which determines the probability density that $N$ interacting molecules are found on $\left(\boldsymbol{r}_{1}^{1}, \ldots, \boldsymbol{r}_{M}^{N}\right)$ in the system box with the volume $V$ equilibrated with the reservoir $\mu$ and $T$. This probability density is denoted by $\hat{w}_{N}\left(\boldsymbol{r}_{1}^{1}, \ldots, \boldsymbol{r}_{M}^{N}\right)$.

$$
\hat{w}_{N}\left(\boldsymbol{r}_{1}^{1}, \ldots, \boldsymbol{r}_{M}^{N}\right)=\frac{1}{\Xi(\mu, T, V)} \frac{1}{\Lambda^{3 N M} N !} \exp \left[-\frac{1}{k_{B} T} U\left(\boldsymbol{r}_{1}^{1}, \ldots, \boldsymbol{r}_{M}^{N}\right)+\frac{\mu}{k_{B} T} N\right]
$$

The probability density of $N$ is calculated from (3.26):

$$
\begin{aligned}
& H_{\mu, T, V}(N):=\int d \boldsymbol{r}_{1}^{1} \ldots d \boldsymbol{r}_{M}^{N} \hat{w}_{N}\left(\boldsymbol{r}_{1}^{1}, \ldots, \boldsymbol{r}_{M}^{N}\right) \\
& =\frac{1}{\Xi(\mu, T, V)} \frac{1}{\Lambda^{3 N M} N !} \exp \left[\frac{\mu}{k_{B} T} N\right] \int d \boldsymbol{r}_{1}^{1} \ldots d \boldsymbol{r}_{M}^{N} \exp \left[-\frac{1}{k_{B} T} U\left(\boldsymbol{r}_{1}^{1}, \ldots, \boldsymbol{r}_{M}^{N}\right)\right]
\end{aligned}
$$

\subsection{Configurational-bias method in $\mu V T$-ensemble}

In grand canonical ensemble, $\mu V T$-ensemble, the system box and the reservoir exchange particles. In simulation in $\mu V T$-ensemble, particles are inserted into and deleted from the system in addition to Metropolis trial movement. In one simulation step:

i) with probability $p_{g}$, trial particle insertion into the system

ii) with probability $p_{g}$, trial particle deletion from the system

iii) with probability $1-2 p_{g}$, trial movement based on Metropolis algorithm i.e. perturbation of one segment 
is chosen.

When one macromolecule is inserted in the system box at random, segments of this extended molecule overlap with others with a high probability and this trial is rejected. In configurational-bias method the inserted molecule is built segment by segment. The bias in constructing the molecule reduces the probability of the overlap between the molecules. We will discuss this configurational-bias method in grand canonical ensemble. In the present formulation, the reference state in the chemical potential (see eq. (3.17)), $j(T)$, and thermal de Broglie wave length $\Lambda$ are absorbed in the definition of the chain potential (cf. original articles [50, 54]); we can simulate any systems in grand canonical ensemble regardless of the unknown reference state $j(T)$ and $\Lambda$ (see also section 3.4.3).

\subsubsection{Particle insertion}

To insert a molecule into the system via configurational-bias method, we use the following 4 steps.

1) The first atom of the molecule, the end segment, is located in the system box at random. The energy between this atom and the others is defined as $u^{n, \mathrm{nb}}(1)$.

2) In order to link another segment to this end segment and to grow the molecule segment by segment, a set of $k$ trial positions is generated for the $l$-th segment. These positions are denoted by $\left\{\boldsymbol{b}^{l}\right\}=\left(\boldsymbol{b}_{1}^{l}, \boldsymbol{b}_{2}^{l}, \ldots, \boldsymbol{b}_{k}^{l}\right)$. These vectors are drawn from the center of the $(l-1)$-th segment and correspond to the bond vector eq. (3.19) that connect the $(l-1)$-th and the $l$-th segments. This set of trial orientations are generated using the internal potential energy, which results in the distribution:

$$
p_{l}^{\text {int }}\left(\boldsymbol{b}_{i}^{l}\right) d \boldsymbol{b}_{i}^{l}=\frac{\exp \left[-\beta u_{l}^{n, i n t}\left(\boldsymbol{b}_{i}^{l}\right)\right] d \boldsymbol{b}_{i}^{l}}{C_{l}}, \quad 1 \leq i \leq k
$$

where the inverse temperature $\beta=1 / k_{B} T, u_{l}^{n \text { int }}\left(\boldsymbol{b}_{i}^{l}\right)$ denotes the internal potential energy between the trial position located by $\boldsymbol{b}_{i}^{l}$ as the $l$-th segment and the other segments which have already been grown, and $C_{l}$ is a normalization constant. $C_{l}$ is related to the partition function of an ideal molecule, i.e. eq. (3.20),

$$
\begin{aligned}
\frac{V}{\Lambda^{3 M}} \prod_{l=2}^{M} C_{l} & =\frac{V}{\Lambda^{3 M}} \int \exp \left[-\beta \sum_{l=2}^{M} u_{l}^{n \text {,int }}\left(\boldsymbol{b}_{i}^{l}\right)\right] \prod_{l=2}^{M} d \boldsymbol{b}_{i}^{l} \\
& =\frac{V}{\Lambda^{3 M}} \int d \boldsymbol{u}_{1} \ldots d \boldsymbol{u}_{M-1} \exp \left[-\frac{1}{k_{B} T} \tilde{U}^{\text {int }}\left(\boldsymbol{u}_{1}, \ldots, \boldsymbol{u}_{M-1}\right)\right] \\
& =Z_{1}^{\text {ideal }}(T, V)
\end{aligned}
$$

In our present simulation model, $u_{l}^{n \text {,int }}\left(\boldsymbol{b}_{i}^{l}\right)$ is a harmonic spring potential. Therefore $x, y, z$-elements of $\boldsymbol{b}_{i}^{l}$ are independently distributed by the Gaussian distribution with the average, 0 , and the variance, $R_{e}^{2} /\{3(M-1)\}$, where $M$ denotes the number of segments per molecule. 
For each of these trial positions, the non-bonded potential energy between this trial position and the other segments, i.e. the other molecules and the segments which have already been grown in the present molecule, is calculated. This energy is denoted by $u_{l}^{n, n b}\left(\boldsymbol{b}_{j}^{l}\right)$ and one of these positions is selected as the $l$-th segment with a probability

$$
p_{l}^{\mathrm{nb}}\left(\boldsymbol{b}_{j}^{l}\right)=\frac{\exp \left[-\beta u_{l}^{n, n b}\left(\boldsymbol{b}_{j}^{l}\right)\right]}{w^{n, n b}(l)}, \quad 1 \leq j \leq k,
$$

where

$$
w^{n, n b}(l)=\sum_{j=1}^{k} \exp \left[-\beta u_{l}^{n, n b}\left(\boldsymbol{b}_{j}^{l}\right)\right] .
$$

This step 2) is also schematically illustrated in Fig. 3.2. Since we favour conformations with a low energy, we have introduced a bias. For the purpose of satisfying the detailed balance, this bias should be compensated in the acceptance rules.

We define a set of $\left\{\boldsymbol{b}^{l}\right\}$ over the trial molecule as

$$
\{\boldsymbol{B}\}:=\left(\left\{\boldsymbol{b}^{2}\right\}, \ldots,\left\{\boldsymbol{b}^{M}\right\}\right) .
$$

3) After repeating step 2) until the whole molecule with the number of segments per molecule, $M$, has been grown, we calculate the Rosenbluth weight

$$
\mathcal{W}^{n}:=\exp \left[-\beta u^{n, n b}(1)\right] \prod_{l=2}^{M} \frac{w^{n, n b}(l)}{k} .
$$

4) The new molecule is accepted with a probability

$$
\operatorname{acc}(N \rightarrow N+1 \mid\{\boldsymbol{B}\})=\min \left(1, \frac{V \exp [\beta \mu]}{\Lambda^{3}(N+1)} \mathcal{W}^{n} j(T)\right),
$$

where $N$ denotes the current number of molecules in the system. $j(T)$ and the chemical potential of the reservoir consisting of ideal molecules denoted by $\mu$ are given in (3.18) and (3.17) respectively. We can reduce the acceptance criterion by expressing $j$ and $\mu$ in terms of the density $\rho_{\text {res }}$ of a with non-interacting molecules.

$$
\operatorname{acc}(N \rightarrow N+1 \mid\{\boldsymbol{B}\})=\min \left(1, \frac{V}{N+1} \rho_{\text {res }} \mathcal{W}^{n}\right) .
$$

The reference state in the chemical potential, $j(T)$, and thermal de Broglie wave length $\Lambda$ have disappeared from our acceptance criterion.

At large parameter $k$, high acceptance ratio is obtained because energetically good configurations are found. On the other hand, at small $k$, a program runs fast whereas the acceptance ratio is low.

Particle deletion is discussed in appendix B. 


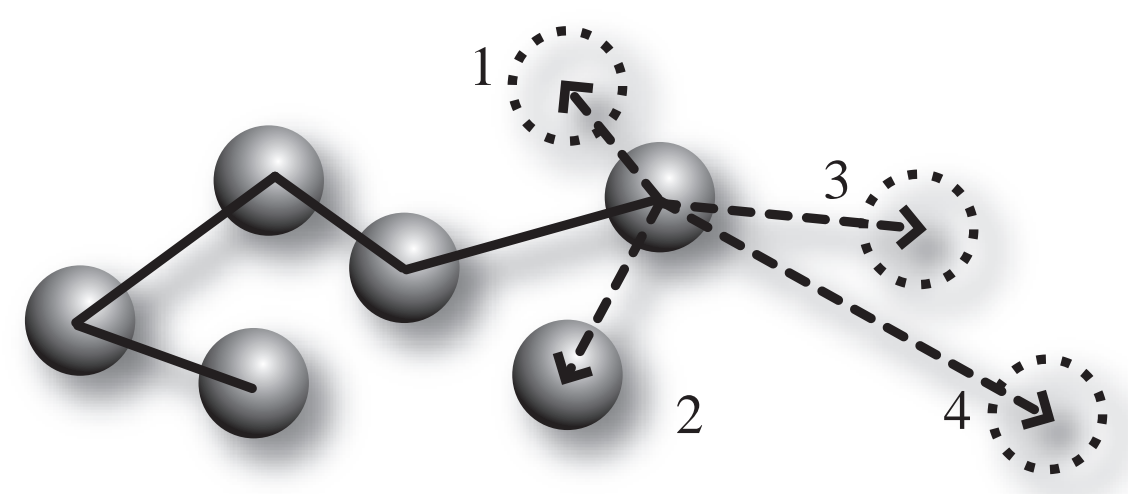

Figure 3.2: Step 2) in the particle insertion. After the first segment is randomly placed in the system, the other segments that belong to the inserted molecule are grown segment by segment. The $l$-th segment is connected to the $(l-1)$-th segment in step 2$)$. In this figure, as an example, at $k=4$ the 6th segment is linked. Solid lines and spheres represent the molecular configuration and the segments already grown respectively. First, $\left\{\boldsymbol{b}^{l}\right\}=$ $\left(\boldsymbol{b}_{1}^{l}, \ldots, \boldsymbol{b}_{i}^{l}, \ldots, \boldsymbol{b}_{k}^{l}\right), l=6$, is generated according to the internal potential energy between the 6th segment as an trial position and the other segments i.e. from the 1st to the 5th. These $k$ trial positions are drawn by broken arrows and broken spheres with the number $i$. One of the trial positions is chosen as the real 6th segment according to the non-bonded potential energy of each trial position. This non-bonded potential energy is calculated between each of the trial 6th segment and the other segments i.e. the other molecules and the segments which have already been built. In this example, the 2 nd trial position, $i=2$, is selected as the 6th segment.

\subsubsection{Detailed balance condition of the configurational-bias method}

When one molecule is inserted on the position $\boldsymbol{r}^{N+1}:=\left(\boldsymbol{r}_{1}^{N+1}, \ldots, \boldsymbol{r}_{M}^{N+1}\right)$ to the particle configuration $\left(\boldsymbol{r}_{1}^{1}, \ldots, \boldsymbol{r}_{M}^{N}\right)$, the probability flow from the state $\left(\boldsymbol{r}_{1}^{1}, \ldots, \boldsymbol{r}_{M}^{N}\right)$ to $\left(r_{1}^{1}, \ldots, r_{M}^{N+1}\right)$ is defined as

$$
K\left(\left(\boldsymbol{r}_{1}^{1}, \ldots, \boldsymbol{r}_{M}^{N} \mid N\right) \rightarrow\left(\boldsymbol{r}_{1}^{1}, \ldots, \boldsymbol{r}_{M}^{N+1} \mid N+1\right)\right)
$$

This flow is a product of the probability that the system is found in the state $\left(r_{1}^{1}, \ldots, r_{M}^{N}\right)$, given in eq. (3.26), and the transition probability in this flow, denoted by

$$
\operatorname{Pr}\left(\left(\boldsymbol{r}_{1}^{1}, \ldots, \boldsymbol{r}_{M}^{N} \mid N\right) \rightarrow\left(\boldsymbol{r}_{1}^{1}, \ldots, \boldsymbol{r}_{M}^{N+1} \mid N+1\right)\right)
$$

The flow is:

$$
\begin{aligned}
& K\left(\left(\boldsymbol{r}_{1}^{1}, \ldots, \boldsymbol{r}_{M}^{N} \mid N\right) \rightarrow\left(\boldsymbol{r}_{1}^{1}, \ldots, \boldsymbol{r}_{M}^{N+1} \mid N+1\right)\right)= \\
& \hat{w}_{N}\left(\boldsymbol{r}_{1}^{1}, \ldots, \boldsymbol{r}_{M}^{N}\right)(d V)^{M N} \times \operatorname{Pr}\left(\left(\boldsymbol{r}_{1}^{1}, \ldots, \boldsymbol{r}_{M}^{N} \mid N\right) \rightarrow\left(\boldsymbol{r}_{1}^{1}, \ldots, \boldsymbol{r}_{M}^{N+1} \mid N+1\right)\right) .
\end{aligned}
$$


In the reverse flow, i.e. the flow where the inserted molecule on $\boldsymbol{r}^{N+1}$ is deleted, is also defined in the same way,

$$
\begin{aligned}
& K\left(\left(\boldsymbol{r}_{1}^{1}, \ldots, \boldsymbol{r}_{M}^{N+1} \mid N+1\right) \rightarrow\left(\boldsymbol{r}_{1}^{1}, \ldots, \boldsymbol{r}_{M}^{N} \mid N\right)\right)= \\
& \hat{w}_{N+1}\left(\boldsymbol{r}_{1}^{1}, \ldots, \boldsymbol{r}_{M}^{N+1}\right)(d V)^{M(N+1)} \times \operatorname{Pr}\left(\left(\boldsymbol{r}_{1}^{1}, \ldots, \boldsymbol{r}_{M}^{N+1} \mid N+1\right) \rightarrow\left(\boldsymbol{r}_{1}^{1}, \ldots, \boldsymbol{r}_{M}^{N} \mid N\right)\right) .
\end{aligned}
$$

Our molecule is constructed segment by segment with a bias. In the following we prove that our acceptance criterion compensates the bias and that our simulation method satisfies the detailed balance in statistical mechanics,

$$
\begin{aligned}
K\left(\left(\boldsymbol{r}_{1}^{1}, \ldots, \boldsymbol{r}_{M}^{N} \mid N\right) \rightarrow\left(\boldsymbol{r}_{1}^{1}, \ldots, \boldsymbol{r}_{M}^{N+1} \mid N+1\right)\right)= \\
K\left(\left(\boldsymbol{r}_{1}^{1}, \ldots, \boldsymbol{r}_{M}^{N+1} \mid N+1\right) \rightarrow\left(\boldsymbol{r}_{1}^{1}, \ldots, \boldsymbol{r}_{M}^{N} \mid N\right)\right) .
\end{aligned}
$$

Hereafter for simplicity, we denote the states only with the number of the molecules, e.g.

$$
(N):=\left(r_{1}^{1}, \ldots, r_{M}^{N} \mid N\right)
$$

\subsection{2-a Flow of probability in the step insertion}

First we calculate the flow (3.35). In this equation, the former factor, $\hat{w}_{N}\left(\boldsymbol{r}_{1}^{1}, \ldots, \boldsymbol{r}_{M}^{N}\right)$, has already been given in eq. (3.26). From now we will determine the latter factor, the transition probability.

The first atom of the inserted molecule, the end segment, is located on $\boldsymbol{r}_{1}^{N+1}$ with probability

$$
\frac{1}{V} d V
$$

For $l$-th segment, we assume that the trial molecule is arranged on $\boldsymbol{r}_{l}^{N+1}$ when a trial orientation $\boldsymbol{b}_{\Gamma}^{l}$ is generated and chosen. A set $\left\{\boldsymbol{b}^{l}\right\}$ including such $\boldsymbol{b}_{\Gamma}^{l}$ is denoted by $\left\{\boldsymbol{b}^{\Gamma l}\right\}$. A set of $\left\{\boldsymbol{b}^{\Gamma l}\right\}$ over the trial molecule is defined as

$$
\left\{\boldsymbol{B}^{\Gamma}\right\}:=\left(\left\{\boldsymbol{b}^{\Gamma 2}\right\}, \ldots,\left\{\boldsymbol{b}^{\Gamma M}\right\}\right) .
$$

The probability that the trial position $\boldsymbol{r}^{N+1}$ is generated with a $\{\boldsymbol{B}\}\left(\in\left\{\boldsymbol{B}^{\Gamma}\right\}\right)$ is:

$$
\frac{1}{V} d V \prod_{l=2}^{M}\left\{\frac{\exp \left[-\beta u_{l}^{n, n b}\left(\boldsymbol{b}_{\Gamma}^{l}\right)\right]}{w^{n, n b}(l)} \prod_{i=1}^{k} \frac{\exp \left[-\beta u_{l}^{n, i n t}\left(\boldsymbol{b}_{i}^{l}\right)\right] d V}{C_{l}}\right\}
$$

This trial is accepted with the acceptance criterion

$$
\operatorname{acc}(N \rightarrow N+1 \mid\{\boldsymbol{B}\}) .
$$


The total probability to generate and accept this trial insertion on $\boldsymbol{r}^{N+1}$, i.e. the transition probability, is obtained via summation of these relations over all the $\{\boldsymbol{B}\}\left(\in\left\{\boldsymbol{B}^{\Gamma}\right\}\right)$ :

$$
\begin{aligned}
\operatorname{Pr}(N \rightarrow N+1)= & \\
\frac{1}{V} d V \sum_{\{\boldsymbol{B}\} \in\left\{\boldsymbol{B}^{\Gamma}\right\}} & {\left[\prod _ { l = 2 } ^ { M } \left\{\frac{\exp \left[-\beta u_{l}^{n, n b}\left(\boldsymbol{b}_{\Gamma}^{l}\right)\right]}{w^{n, n b}(l)}\right.\right.} \\
& \left.\left.k \prod_{i=1}^{k} \frac{\exp \left[-\beta u_{l}^{n, i n t}\left(\boldsymbol{b}_{i}^{l}\right)\right] d V}{C_{l}}\right\} \operatorname{acc}(N \rightarrow N+1 \mid\{\boldsymbol{B}\})\right] .
\end{aligned}
$$

Note that the factor $k$ means that the position of $\boldsymbol{b}_{\Gamma}^{l}$ in $\left\{\boldsymbol{b}^{l}\right\}$ does not matter; hereafter $\boldsymbol{b}_{\Gamma}^{l}$ is fixed on the last of $\left\{\boldsymbol{b}^{l}\right\}$, i.e. $\left\{\boldsymbol{b}^{\Gamma l}\right\}=\left(\boldsymbol{b}_{1}^{l}, \ldots, \boldsymbol{b}_{k-1}^{l}, \boldsymbol{b}_{\Gamma}^{l}\right)$. This transition probability (3.38) yields the flow (3.35) =

$$
\begin{aligned}
K(N \rightarrow N+1)= & \hat{w}_{N}\left(\boldsymbol{r}_{1}^{1}, \ldots, \boldsymbol{r}_{M}^{N}\right)(d V)^{M N} \times \operatorname{Pr}(N \rightarrow N+1) \\
= & \frac{1}{\Xi(\mu, T, V)} \frac{1}{\Lambda^{3 N M} N !} \exp \left[-\beta U\left(\boldsymbol{r}_{1}^{1}, \ldots, \boldsymbol{r}_{M}^{N}\right)+\beta \mu N\right](d V)^{M N} \\
& \times \frac{1}{V} d V \sum_{\{\boldsymbol{B}\} \in\left\{\boldsymbol{B}^{\Gamma}\right\}}\left[\prod _ { l = 2 } ^ { M } \left\{\frac{\exp \left[-\beta u_{l}^{n, n b}\left(\boldsymbol{b}_{\Gamma}^{l}\right)\right]}{w^{n, n b}(l)}\right.\right. \\
& \left.\left.k \prod_{i=1}^{k} \frac{\exp \left[-\beta u_{l}^{n, i n t}\left(\boldsymbol{b}_{i}^{l}\right)\right] d V}{C_{l}}\right\} \operatorname{acc}(N \rightarrow N+1 \mid\{\boldsymbol{B}\})\right] .
\end{aligned}
$$

\subsection{2-b Flow of the probability in the deletion step}

The reverse flow (3.36) is also calculated in the similar way to (3.39).

$$
\begin{aligned}
& K(N+1 \rightarrow N)=\hat{w}_{N+1}\left(\boldsymbol{r}_{1}^{1}, \ldots, \boldsymbol{r}_{M}^{N+1}\right)(d V)^{M(N+1)} \times \operatorname{Pr}(N+1 \rightarrow N) \\
& =\frac{1}{\Xi(\mu, T, V)} \frac{1}{\Lambda^{3(N+1) M}(N+1) !} \exp \left[-\beta U\left(\boldsymbol{r}_{1}^{1}, \ldots, \boldsymbol{r}_{M}^{N+1}\right)+\beta \mu(N+1)\right](d V)^{M(N+1)} \\
& \quad \times \sum_{\{\boldsymbol{B}\} \in\left\{\boldsymbol{B}^{\Gamma}\right\}}\left[\prod_{l=2}^{M}\left\{\prod_{i=1}^{k-1} \frac{\exp \left[-\beta u_{l}^{0, i n t}\left(\boldsymbol{b}_{i}^{l}\right)\right] d V}{C_{l}}\right\} \operatorname{acc}(N+1 \rightarrow N \mid\{\boldsymbol{B}\})\right] .
\end{aligned}
$$

\subsection{2-c Detailed balance in configurational-bias method}

In order to satisfy the detailed balance (3.37), the flow (3.39) should equal the reverse flow (3.40). This detailed balance is certainly fulfilled if each term in (3.39), for a specic choice of trial vectors $\{\boldsymbol{B}\}\left(\in\left\{\boldsymbol{B}^{\Gamma}\right\}\right)$, is equal to its counterpart in (3.40), which corresponds to the same $\{\boldsymbol{B}\}$. This stronger condition is called super-detailed 
balance $[50,54]$. Imposing this super-detailed balance, we determine the acceptance criterion acc $(N \rightarrow N+1 \mid\{\boldsymbol{B}\})$.

Super-detailed balance and the equations (3.37), (3.39), (3.40), (3.32), (3.29), and (3.18) yield the relation

$$
\operatorname{acc}(N \rightarrow N+1 \mid\{\boldsymbol{B}\})=\frac{V \exp [\beta \mu]}{\Lambda^{3}(N+1)} \mathcal{W}^{n} j(T) \operatorname{acc}(N+1 \rightarrow N \mid\{\boldsymbol{B}\}) .
$$

When

$$
\frac{V \exp [\beta \mu]}{\Lambda^{3}(N+1)} W^{n} j(T)<1,
$$

one choice for the acceptance criteria that fulfill the condition (3.41) is:

$$
\operatorname{acc}(N \rightarrow N+1 \mid\{\boldsymbol{B}\})=\frac{V \exp [\beta \mu]}{\Lambda^{3}(N+1)} \mathcal{W}^{n} j(T), \quad \operatorname{acc}(N+1 \rightarrow N \mid\{\boldsymbol{B}\})=1 .
$$

When

$$
\frac{V \exp [\beta \mu]}{\Lambda^{3}(N+1)} W^{n} j(T) \geq 1
$$

due to $\mathcal{W}^{n}=\mathcal{W}^{0}$ for the same $\{\boldsymbol{B}\}$,

$$
\operatorname{acc}(N \rightarrow N+1 \mid\{\boldsymbol{B}\})=1, \quad \operatorname{acc}(N+1 \rightarrow N \mid\{\boldsymbol{B}\})=\frac{\Lambda^{3}(N+1)}{V \exp [\beta \mu]} \frac{1}{W^{0}} \frac{1}{j(T)}
$$

These results (3.42) and (3.43) are consistent with the acceptance criteria (3.33) and (B.3). Thus our method satisfies the detailed balance.

\subsubsection{Test simulation of the configurational-bias method}

As a test on our configurational-bias method and simulation program, we simulate ideal polymers and single component solvent-free homopolymers. In this section, $N$ denotes the number of segments per polymer and $n_{p}$ the number of the polymers in the system. $N$ is fixed at 32 . In these tests, $1 \%$ of the total simulation steps is on average chosen for the particle exchange between the system and the reservoir. The number of trial positions in constructing one atom, denoted by $k$ in our algorithm (see section 3.3.1), is fixed at 5 .

\subsection{3-a Test simulation on ideal polymers in $\mu V T$-ensemble}

First we simulate ideal polymers via our configurational-bias method in the grand canonical ensemble. In our simulation, $V$ is fixed at $2.02^{3} R_{e}^{3}$ and the dimensionless polymer density of the reservoir, $\rho_{\text {res }}^{\prime}$, is 122 .

$$
\log \rho_{\text {res }}^{\prime}=\beta \mu_{\text {ideal }}\left(T, \rho_{\text {res }}^{\prime}\right)-\log \left(\Lambda^{3} /\left(R_{e}^{3} Z_{0}\right)\right)=4.80, \quad \text { see eq. (3.23). }
$$


Since our system consists of the identical ideal polymers which also compose the reservoir, the average polymer density in the system is equal to the one in the reservoir. In equilibrium, the average number of the polymers in the system is

$$
\left\langle n_{p}\right\rangle=V \rho_{\text {res }}^{\prime}=1000 \text {. }
$$

In the initial configuration, $n_{p 0}$ polymers are arranged in the cubic system box. Figure 3.3 shows the number of the polymers in the system box, $n_{p}$, at the initial values $n_{p 0}=500,1000$, and 1728 . These data indicate that $n_{p}$ fluctuates around $\left\langle n_{p}\right\rangle=1000$. This simulation result is consistent with our discussion.

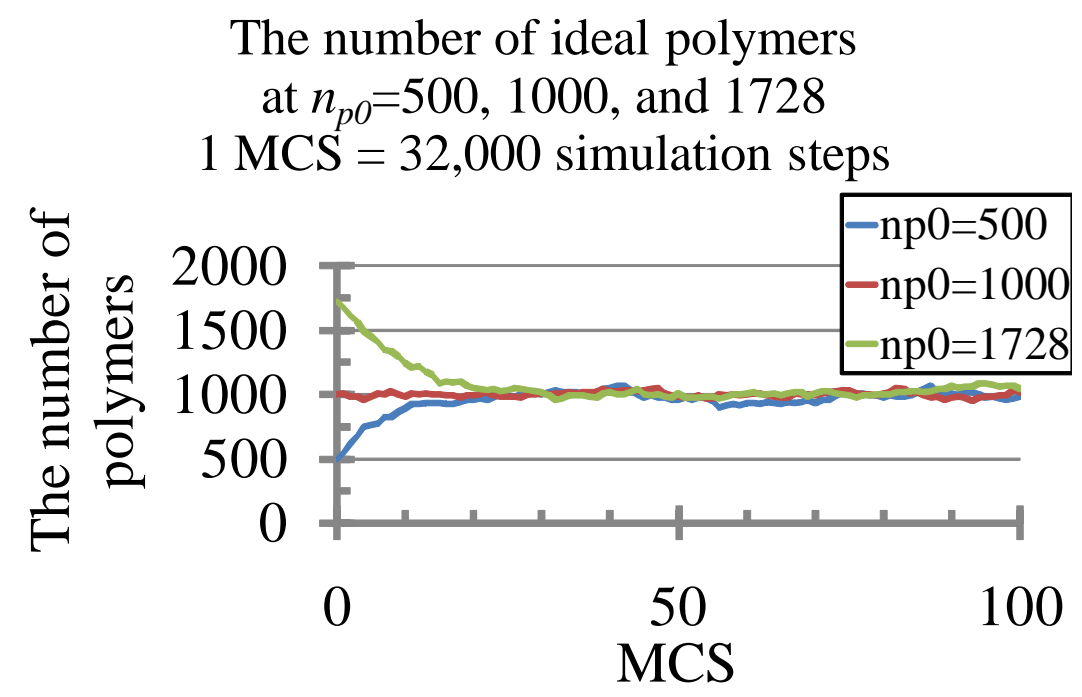

Figure 3.3: The number of polymers, $n_{p}$, sampled every MCS, where 1 MCS $:=32,000$ simulation steps. Simulations are started at the initial number of polymers $n_{p 0}=500,1000$, and 1728 respectively.

\subsection{3-b Single component solvent-free system in $\mu V T$-ensemble}

In our single component solvent-free homopolymer model (see section 2.1), the free energy of the system is defined as

$$
\frac{F\left(T, V, n_{p}\right)}{k_{B} T}=\frac{F_{\text {ideal }}\left(T, V, n_{p}\right)}{k_{B} T}+\frac{F_{\text {excess }}\left(T, V, n_{p}\right)}{k_{B} T},
$$

where $F_{\text {ideal }}$ is given in (3.22) and (3.24) and the excess free energy

$$
\begin{aligned}
\frac{F_{\text {excess }}\left(T, V, n_{p}\right)}{k_{B} T} & =\int_{V} d V\left(-\frac{1}{2} v \rho_{s}^{2}+\frac{1}{3} w \rho_{s}^{3}\right) \\
& \cong \sum_{\text {cells }}\left\{\Delta V\left(-\frac{1}{2} v \rho_{s}^{2}+\frac{1}{3} w \rho_{s}^{3}\right)\right\} .
\end{aligned}
$$


Table 3.1: Simulation parameters for the test simulation of single-component solvent-free homopolymers on configurational-bias method in grand canonical ensemble. $R_{e}$ is taken as the unit length.

$$
\begin{aligned}
\text { Polymerization degree } N & =32 \\
\text { System size } L & =2.0 \\
v^{\prime} & =0.01 \\
w^{\prime} & =0.0001
\end{aligned}
$$

The width of the cell $\Delta L \approx 0.166$

The maximum trial displacement $=\Delta L$

Mean-field approximation yields

$$
\frac{F_{\mathrm{excess}}\left(T, V, n_{p}\right)}{k_{B} T} \approx V\left(-\frac{1}{2} v \rho_{s}^{2}+\frac{1}{3} w \rho_{s}^{3}\right)
$$

In this approximation, the total chemical potential of the system is:

$$
\frac{\mu\left(T, \rho_{p}\right)}{k_{B} T}=\log \left(\Lambda^{3} \rho_{p}\right)-\log Z_{0}-v N^{2} \rho_{p}+w N^{3} \rho_{p}^{2}
$$

where $Z_{0}$ has been given in eq. (3.24). With use of the dimensionless parameters, this chemical potential is reduced to

$$
\frac{\mu\left(T, \rho_{p}^{\prime}\right)}{k_{B} T}=\log \left(\frac{\Lambda^{3}}{R_{e}^{3}} \rho_{p}^{\prime}\right)-\log Z_{0}-v^{\prime} \rho_{p}^{\prime}+w^{\prime} \rho_{p}^{\prime 2},
$$

within the mean-field approximation. In equilibrium, $\mu\left(T, \rho_{p}^{\prime}\right)$ should equal the chemical potential of the reservoir given in eq. (3.23). This relation yields

$$
\log \left(\frac{\rho_{p}^{\prime}}{\rho_{\text {res }}^{\prime}}\right)-v^{\prime} \rho_{p}^{\prime}+w^{\prime} \rho_{p}^{\prime 2}=0 .
$$

This equation determines the average $\rho_{p}^{\prime}$ in the system in equilibrium with the reservoir.

Simulation parameters are shown in Table 3.1. This system is simulated at $\rho_{\text {res }}^{\prime}=51,171,200$, and 2646 which correspond to $\rho_{p}^{\prime}=64,125,132$, and 216 respectively (see Table 3.2) via eq. (3.45). Due to the smaller $v^{\prime}$ than the critical value $v_{c}^{\prime}=2 \sqrt{2 w^{\prime}} \cong 0.028$, the system is in the homogeneous phase. In the initial state, each polymer with a Gaussian distribution, i.e. $\left\langle R_{e}^{2}\right\rangle=1$, is distributed in the cubic system box at random.

Figures 3.4, 3.5 and 3.6 show $n_{p}$ at $\rho_{\text {res }}^{\prime}=51,171$, and 2646 respectively. These data fluctuate around $\left\langle n_{p}\right\rangle=\rho_{p}^{\prime} L^{3}=512,1000$, and 1728 respectively. These simulation results correspond to the estimation via mean-field approximation discussed in this section. 
Table 3.2: $\rho_{\text {res }}^{\prime}$ and corresponding $\rho_{p}^{\prime}$ via mean-field approximation. $\left\langle n_{p}\right\rangle:=\rho_{p}^{\prime} L^{3}$ and $\left(\log \rho_{\text {res }}^{\prime}\right)=\beta \mu_{\text {ideal }}\left(T, \rho_{\text {res }}^{\prime}\right)-\log \left(\Lambda^{3} /\left(R_{e}^{3} Z_{0}\right)\right)$, see eq. (3.23).

\begin{tabular}{rrrc}
\hline$\rho_{\text {res }}^{\prime}$ & $\rho_{p}^{\prime}$ & $\left\langle n_{p}\right\rangle$ & $\log \rho_{\text {res }}^{\prime}$ \\
\hline 51 & 64 & 512 & 3.93 \\
171 & 125 & 1000 & 5.14 \\
200 & 132 & 1056 & 5.30 \\
2646 & 216 & 1728 & 7.88 \\
\hline
\end{tabular}

The number of polymers

at $n_{p 0}=256,512$, and 1000

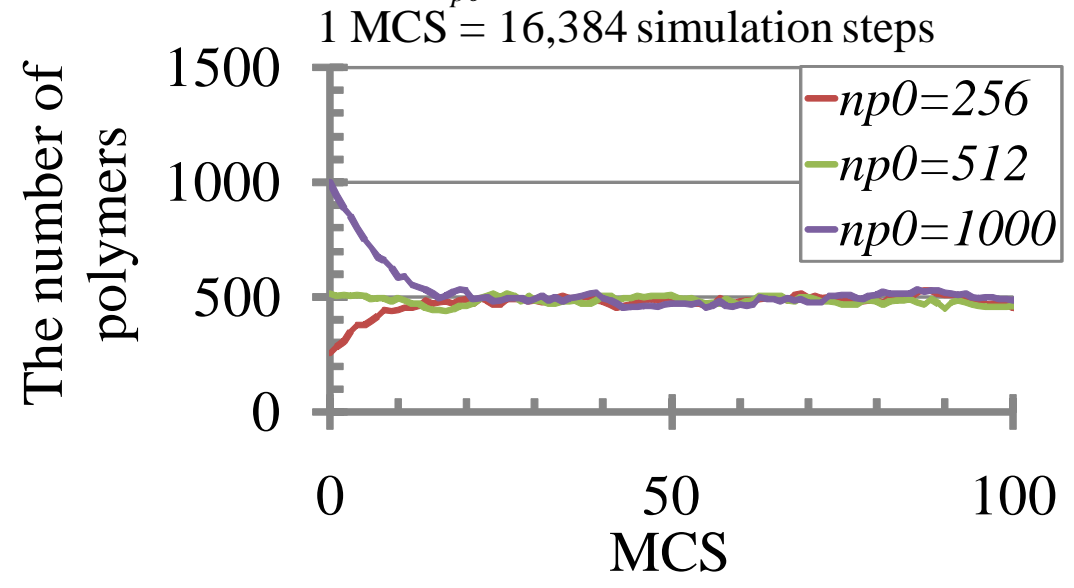

Figure 3.4: The number of polymers $n_{p}$ at $\rho_{\text {res }}^{\prime}=51$ sampled every MCS, where 1 MCS is defined as 16,384 simulation steps. Simulations are started at the initial number of polymers $n_{p 0}=256,512$, and 1000 respectively. The data fluctuate around $\left\langle n_{p}\right\rangle=\rho_{p}^{\prime} L^{3}=$ 512 .
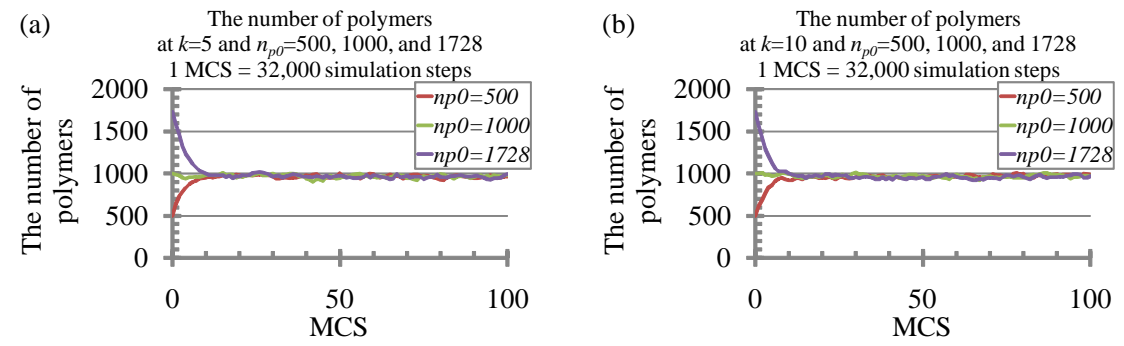

Figure 3.5: The number of polymers $n_{p}$ at $\rho_{\text {res }}^{\prime}=171$ sampled every MCS, where 1 MCS is defined as 32,000 simulation steps. (a): $k=5$ and (b): $k=10$. Simulations are started at the initial number of polymers $n_{p 0}=500,1000$, and 1728 respectively. The data fluctuate around $\left\langle n_{p}\right\rangle=\rho_{p}^{\prime} L^{3}=1000$. 


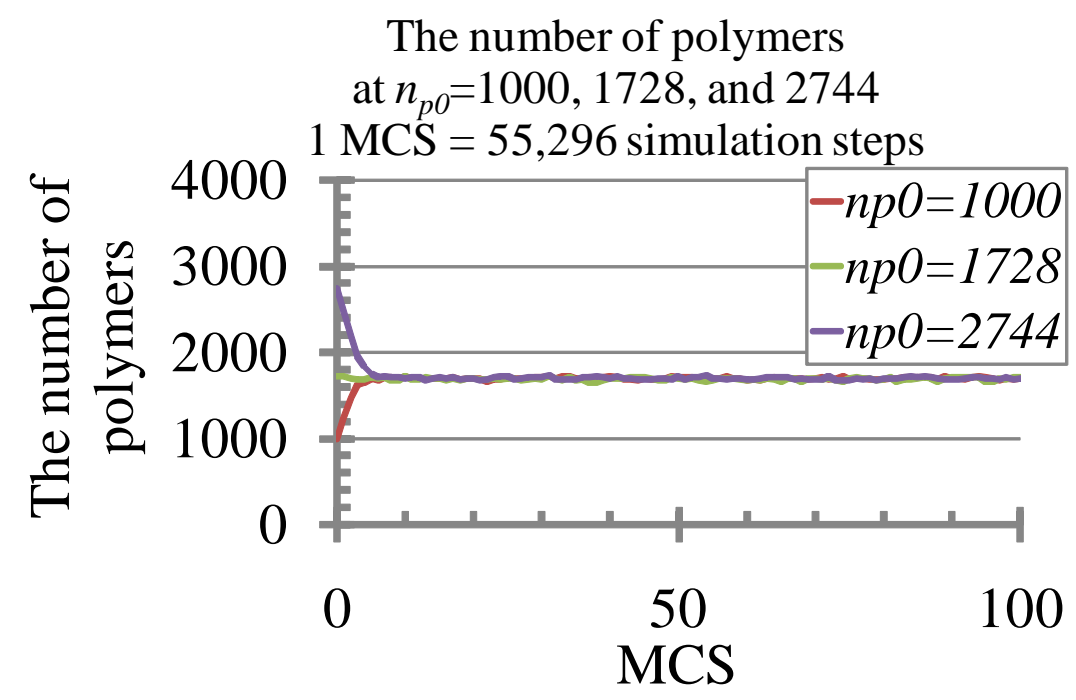

Figure 3.6: The number of polymers $n_{p}$ at $\rho_{\text {res }}^{\prime}=2646$ sampled every MCS, where 1 MCS is defined as 55,296 simulation steps. Simulations are started at the initial number of polymers $n_{p 0}=1000,1728$, and 2744 respectively. The data fluctuate around $\left\langle n_{p}\right\rangle=$ $\rho_{p}^{\prime} L^{3}=1728$.

Next we calculate the probability density of $n_{p}$, given in eq. (3.27), at $\rho_{\text {res }}^{\prime}=$ 171 and 200. The difference in chemical potential between these two systems is denoted by $\Delta \mu$ (see Table 3.2),

$$
\beta \Delta \mu=\log 200-\log 171=0.157 .
$$

Equation (3.27) yield the relation

$$
\frac{H_{\mu+\Delta \mu, T, V}\left(n_{p}\right)}{H_{\mu, T, V}\left(n_{p}\right)} \exp \left[-\frac{\Delta \mu}{k_{B} T} n_{p}\right]=\frac{\Xi(\mu, T, V)}{\Xi(\mu+\Delta \mu, T, V)} .
$$

In Fig. 3.7, these probability densities and the left-hand side of eq. (3.46) as a function of $n_{p}$ are plotted. The flat graph of this quantity is consistent with the relation (3.46).

These test simulations indicate the validity of our configurational-bias method and the simulation program. 


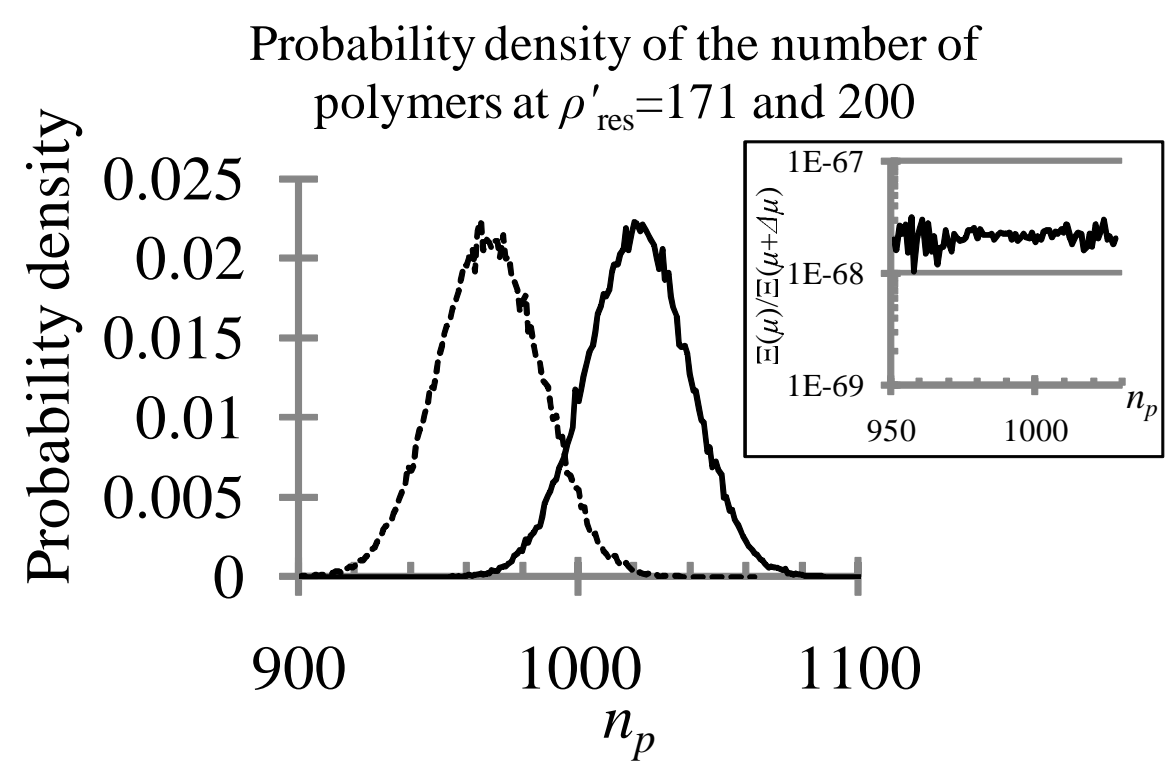

Figure 3.7: Probability density of $n_{p}$ at $\rho_{\text {res }}^{\prime}=171$ and 200 plotted by a broken line and a solid line respectively. Simulations are started at the initial number of polymers $n_{p 0}=\left\langle n_{p}\right\rangle$. Data are sampled every 10 MCS and 70,000 independent samples are obtained, where 1 MCS $:=33,792$ simulation steps at $\rho_{\text {res }}^{\prime}=200$. The left-hand side of eq. (3.46) as a function of $n_{p}$ is plotted in the inset. 


\subsection{Chemical potential in $N V T$-ensemble}

We also measure the chemical potential of the macromolecular system in the canonical ensemble.

In the canonical ensemble, the chemical potential of the system $\mu(T, V, N)$ is determined by the partition function (3.6) and (3.10):

$$
\begin{aligned}
\beta \mu(T, V, N) & =-\frac{\partial}{\partial N} \log Z_{N}(T, V) \\
& =-\frac{\partial}{\partial N} \log Z_{N}^{\text {ideal }}(T, V)-\frac{\partial}{\partial N} \log \left(\frac{Z_{N}(T, V)}{Z_{N}^{\text {ideal }}(T, V)}\right) \\
& =\beta \mu_{\text {ideal }}(T, V, N)+\beta \mu_{\text {excess }}(T, V, N) \\
& =\log \left(\Lambda^{3} \rho\right)-\log j(T)+\beta \mu_{\text {excess }}(T, V, N) .
\end{aligned}
$$

Chemical potential of ideal molecules, $\mu_{\text {ideal }}(T, V, N)$, is defined in eq. (3.17). The physical quantity $\mu_{\text {excess }}(T, V, N)$ corresponds to the excess chemical potential due to the interaction potential between the atoms:

$$
\begin{aligned}
\beta \mu_{\text {excess }}(T, V, N) & :=-\frac{\partial}{\partial N} \log \left(\frac{Z_{N}(T, V)}{Z_{N}^{\text {ideal }}(T, V)}\right) \\
& \cong-\frac{1}{(N+1)-N}\left[\log \left(\frac{Z_{N+1}(T, V)}{Z_{N+1}^{\text {ideal }}(T, V)}\right)-\log \left(\frac{Z_{N}(T, V)}{Z_{N}^{\text {ideal }}(T, V)}\right)\right] \\
& =-\log \left(\frac{(N+1) Z_{N+1}(T, V)}{Z_{N}(T, V) Z_{1}^{\text {ideal }}(T, V)}\right)
\end{aligned}
$$

We discuss the method of calculating this excess chemical potential.

Note that $\Lambda$ and $j(T)$ in chemical potential are never determined in our simulation. However this issue, discussed in section 3.4.3, does not affect our simulation methods and results.

\subsubsection{Widom's insertion and deletion methods}

We calculate the chemical potential of our system via the combination method of Widom's particle insertion and deletion methods [55] invented for monatomic particles by Shing and Gubbins [56,57]. Here we develop their method to our macromolecules. In this method, molecules are inserted and deleted without any bias. 


\subsection{1-a Probability density determined without bias}

Excess chemical potential eq. (3.48) is calculated by the particle deletion without any bias:

$$
\begin{aligned}
& \beta \mu_{\text {excess }}(T, V, N) \\
& =-\log \left(\frac{(N+1) Z_{N+1}(T, V)}{Z_{N}(T, V) Z_{1}^{\text {ideal }}(T, V)}\right) \\
& =\log \left(\frac{1}{Z_{N+1}(T, V)} \frac{1}{\Lambda^{3(N+1) M}(N+1) !} \int d \boldsymbol{r}_{1}^{1} \ldots d \boldsymbol{r}_{M}^{N+1} \exp \left[-\frac{1}{k_{B} T} U\left(\boldsymbol{r}_{1}^{1}, \ldots, \boldsymbol{r}_{M}^{N}\right)\right]\right. \\
& \left.\quad \quad \times \exp \left[-\frac{1}{k_{B} T} U^{\text {int }}\left(\boldsymbol{r}_{1}^{N+1}, \ldots, \boldsymbol{r}_{M}^{N+1}\right)\right]\right) \\
& =\log \left\langle\exp \left[\frac{1}{k_{B} T} U_{\text {excess }}^{\mathrm{nb}}\left(\boldsymbol{r}_{1}^{1}, \ldots, \boldsymbol{r}_{M}^{N} \mid \boldsymbol{r}_{1}^{N+1}, \ldots, \boldsymbol{r}_{M}^{N+1}\right)\right]\right\rangle_{T, V, N+1},
\end{aligned}
$$

where $U_{\text {excess }}^{\mathrm{nb}}\left(\boldsymbol{r}_{1}^{1}, \ldots, \boldsymbol{r}_{M}^{N} \mid \boldsymbol{r}_{1}^{N+1}, \ldots, \boldsymbol{r}_{M}^{N+1}\right)$ is the non-bonded potential energy between $(N+1)$-th molecule and the others in the system box. This equation means that a real molecule is deleted from the system. On the other hand, the excess chemical potential is also calculated by the particle insertion without any bias:

$$
\begin{aligned}
& \beta \mu_{\text {excess }}(T, V, N) \\
&=-\log (\left.\frac{(N+1) Z_{N+1}(T, V)}{Z_{N}(T, V) Z_{1}^{\text {ideal }}(T, V)}\right) \\
&=-\log ( \frac{1}{Z_{N}(T, V) Z_{1}^{\text {ideal }}(T, V)} \frac{1}{\Lambda^{3 N M} N !} \int d \boldsymbol{r}_{1}^{1} \ldots d \boldsymbol{r}_{M}^{N} \exp \left[-\frac{1}{k_{B} T} U\left(\boldsymbol{r}_{1}^{1}, \ldots, \boldsymbol{r}_{M}^{N}\right)\right] \\
& \times \frac{1}{\Lambda^{3 M}} \int d \boldsymbol{r}_{1}^{N+1} \ldots d \boldsymbol{r}_{M}^{N+1} \exp \left[-\frac{1}{k_{B} T} U^{\mathrm{int}}\left(\boldsymbol{r}_{1}^{N+1}, \ldots, \boldsymbol{r}_{M}^{N+1}\right)\right] \\
&=-\log \left(\frac{1}{Z_{1}^{\text {ideal }}(T, V)} \frac{1}{\Lambda^{3 M}} \int d \boldsymbol{r}_{1}^{N+1} \ldots d \boldsymbol{r}_{M}^{N+1} \exp \left[-\frac{1}{k_{B} T} U^{\text {int }}\left(\boldsymbol{r}_{1}^{N+1}, \ldots, \boldsymbol{r}_{M}^{N+1}\right)\right]\right. \\
&\left.\quad \times\left\langle\exp \left[-\frac{1}{k_{B} T} U_{\text {excess }}^{\mathrm{nb}}\left(\boldsymbol{r}_{1}^{1}, \ldots, \boldsymbol{r}_{M}^{N} \mid \boldsymbol{r}_{1}^{N+1}, \ldots, \boldsymbol{r}_{M}^{N+1}\right)\right]\right\rangle_{T, V, N}\right) \\
&=-\log \left(\left.\left\langle\exp \left[-\frac{1}{k_{B} T} U_{\text {excess }}^{\mathrm{nb}}\left(\boldsymbol{r}_{1}^{1}, \ldots, \boldsymbol{r}_{M}^{N} \mid \boldsymbol{r}_{1}, \ldots, \boldsymbol{r}_{M}\right)\right]\right\rangle_{T, V, N}\right|_{V, \text { ideal }} ^{\boldsymbol{r}_{1}, \ldots, \boldsymbol{r}_{M}},\right.
\end{aligned}
$$

where $\left(\boldsymbol{r}_{1}, \ldots, \boldsymbol{r}_{M}\right)$ denotes the particle coordinates of $(N+1)$-th molecule distributed by $Z_{1}^{\text {ideal }}(T, V)$ and $\langle\cdots\rangle_{V \text {, ideal }}^{\boldsymbol{r}_{1}, \ldots, \boldsymbol{r}_{M}}$ is the ensemble average of this ideal molecule. This inserted molecule is called a ghost molecule, because it is not a part of the system. This means that the molecule is randomly inserted into the system box 
according to the partition function $Z_{1}^{\text {ideal }}(T, V)$ as ghost molecules and that the excess non-bonded potential energy between this ghost and the others in the system is measured.

Arguments of logarithmic functions in eqns. (3.49) and (3.50) are determined with use of probability density functions, $g_{N+1}\left(U_{\text {excess }}^{\text {nb }}\right)$ and $f_{N}\left(U_{\text {excess }}^{\text {nb }}\right)$, of deleted and inserted molecular energy:

$$
\begin{aligned}
& \left\langle\exp \left[\frac{1}{k_{B} T} U_{\mathrm{excess}}^{\mathrm{nb}}\left(\boldsymbol{r}_{1}^{1}, \ldots, \boldsymbol{r}_{M}^{N} \mid \boldsymbol{r}_{1}^{N+1}, \ldots, \boldsymbol{r}_{M}^{N+1}\right)\right]\right\rangle_{T, V, N+1} \\
& \quad=\int g_{N+1}\left(U_{\mathrm{excess}}^{\mathrm{nb}}\right) \exp \left[\frac{1}{k_{B} T} U_{\mathrm{excess}}^{\mathrm{nb}}\right] d U_{\mathrm{excess}}^{\mathrm{nb}} \\
& \left\langle\left.\left\langle\exp \left[-\frac{1}{k_{B} T} U_{\mathrm{excess}}^{\mathrm{nb}}\left(\boldsymbol{r}_{1}^{1}, \ldots, \boldsymbol{r}_{M}^{N} \mid \boldsymbol{r}_{1}, \ldots, \boldsymbol{r}_{M}\right)\right]\right\rangle_{T, V, N}\right|_{V, \text { ideal }} ^{\boldsymbol{r}_{1}, \ldots, \boldsymbol{r}_{M}}\right. \\
& =\int f_{N}\left(U_{\mathrm{excess}}^{\mathrm{nb}}\right) \exp \left[-\frac{1}{k_{B} T} U_{\mathrm{excess}}^{\mathrm{nb}}\right] d U_{\mathrm{excess}}^{\mathrm{nb}},
\end{aligned}
$$

where

$$
\begin{aligned}
& \begin{array}{l}
g_{N+1}\left(U_{\text {excess }}^{\mathrm{nb}}\right) \\
=\frac{1}{Z_{N+1}(T, V)} \frac{1}{\Lambda^{3(N+1) M}(N+1) !} \int d \boldsymbol{r}_{1}^{1} \ldots d \boldsymbol{r}_{M}^{N+1} \exp \left[-\frac{1}{k_{B} T} U\left(\boldsymbol{r}_{1}^{1}, \ldots, \boldsymbol{r}_{M}^{N+1}\right)\right] \\
\quad \times \delta\left(U_{\mathrm{excess}}^{\mathrm{nb}}\left(\boldsymbol{r}_{1}^{1}, \ldots, \boldsymbol{r}_{M}^{N} \mid \boldsymbol{r}_{1}^{N+1}, \ldots, \boldsymbol{r}_{M}^{N+1}\right)-U_{\text {excess }}^{\mathrm{nb}}\right)
\end{array} \\
& \begin{array}{r}
f_{N}\left(U_{\mathrm{excess}}^{\mathrm{nb}}\right) \\
=\frac{1}{Z_{N}(T, V)} \frac{1}{\Lambda^{3 N M} N !} \int d \boldsymbol{r}_{1}^{1} \ldots d \boldsymbol{r}_{M}^{N} \exp \left[-\frac{1}{k_{B} T} U\left(\boldsymbol{r}_{1}^{1}, \ldots, \boldsymbol{r}_{M}^{N}\right)\right] \\
\quad \times\left\langle\delta\left(U_{\mathrm{excess}}^{\mathrm{nb}}\left(\boldsymbol{r}_{1}^{1}, \ldots, \boldsymbol{r}_{M}^{N} \mid \boldsymbol{r}_{1}, \ldots, \boldsymbol{r}_{M}\right)-U_{\mathrm{excess}}^{\mathrm{nb}}\right)\right\rangle_{V, \text { ideal }}^{\boldsymbol{r}_{1}, \ldots, \boldsymbol{r}_{M}},
\end{array}
\end{aligned}
$$

and $\delta$ is the Dirac delta function.

The ratio between these two probability density functions is related to the ex- 
cess chemical potential via eq. (3.50):

$$
\begin{aligned}
& g_{N+1}(\left.U_{\mathrm{excess}}^{\mathrm{nb}}\right) \\
&=\{\left.\left\langle\left\langle\exp \left[-\frac{1}{k_{B} T} U_{\mathrm{excess}}^{\mathrm{nb}}\left(\boldsymbol{r}_{1}^{1}, \ldots, \boldsymbol{r}_{M}^{N} \mid \boldsymbol{r}_{1}, \ldots, \boldsymbol{r}_{M}\right)\right]\right\rangle_{T, V, N}^{-1}\right\rangle_{V, \text { ideal }}^{\boldsymbol{r}_{1}, \ldots, \boldsymbol{r}_{M}}\right\}^{-1} \\
& \times \frac{1}{Z_{1}^{\mathrm{ideal}}(T, V)} \frac{1}{\Lambda^{3 M}} \int d \boldsymbol{r}_{1}^{N+1} \ldots d \boldsymbol{r}_{M}^{N+1} \exp \left[-\frac{1}{k_{B} T} U^{\mathrm{int}}\left(\boldsymbol{r}_{1}^{N+1}, \ldots, \boldsymbol{r}_{M}^{N+1}\right)\right] \\
& \times \frac{1}{Z_{N}(T, V)} \frac{1}{\Lambda^{3 N M} N !} \int d \boldsymbol{r}_{1}^{1} \ldots d \boldsymbol{r}_{M}^{N} \exp \left[-\frac{1}{k_{B} T} U\left(\boldsymbol{r}_{1}^{1}, \ldots, \boldsymbol{r}_{M}^{N}\right)\right] \\
& \times \exp \left[-\frac{1}{k_{B} T} U_{\mathrm{excess}}^{\mathrm{nb}}\left(\boldsymbol{r}_{1}^{1}, \ldots, \boldsymbol{r}_{M}^{N} \mid \boldsymbol{r}_{1}^{N+1}, \ldots, \boldsymbol{r}_{M}^{N+1}\right)\right] \\
& \times \delta\left(U_{\mathrm{excess}}^{\mathrm{nb}}\left(\boldsymbol{r}_{1}^{1}, \ldots, \boldsymbol{r}_{M}^{N} \mid \boldsymbol{r}_{1}^{N+1}, \ldots, \boldsymbol{r}_{M}^{N+1}\right)-U_{\mathrm{excess}}^{\mathrm{nb}}\right) \\
&\left\langle\left\langle\exp \left[-\frac{1}{k_{B} T} U_{\mathrm{excess}}^{\mathrm{nb}}\left(\boldsymbol{r}_{1}^{1}, \ldots, \boldsymbol{r}_{M}^{N} \mid \boldsymbol{r}_{1}, \ldots, \boldsymbol{r}_{M}\right)\right]\right\rangle_{T, V, N}\right\rangle_{V, \text { ideal }}^{\boldsymbol{r}_{1}, \ldots, \boldsymbol{r}_{M}} \\
&=\exp \left[-\frac{1}{k_{B} T} U_{\mathrm{excess}}^{\mathrm{nb}}\right] f_{N}\left(U_{\mathrm{excess}}^{\mathrm{nb}}\right) \exp \left[\beta \mu_{\mathrm{excess}}(T, V, N)\right] .
\end{aligned}
$$

This equation yields the relation

$$
\beta U_{\mathrm{excess}}^{\mathrm{nb}}+\log \left(\frac{g_{N+1}\left(U_{\mathrm{excess}}^{\mathrm{nb}}\right)}{f_{N}\left(U_{\mathrm{excess}}^{\mathrm{nb}}\right)}\right)=\beta \mu_{\text {excess }}(T, V, N) .
$$

Therefore, the left-hand side of eq. (3.55) plotted as a function of $\beta U_{\text {excess }}^{\mathrm{nb}}$ shows a horizontal line that the vertical element equals the excess chemical potential, $\beta \mu_{\text {excess }}(T, V, N)$.

\subsubsection{Chemical potential calculation via Rosenbluth sampling}

Using the configurational-bias method discussed in section 3.3, we insert a molecule into the system composed of $N$ molecules in the canonical ensemble, i.e. a ghost molecule, and calculate the Rosenbluth weight (3.32). The average Rosenbluth weight calculated in this particle insertion, denoted by $\langle\mathcal{W}\rangle_{T, V, N}$, determines the excess chemical potential $(3.48)[58,59]$ :

$$
\beta \mu_{\text {excess }}(T, V, N)=-\log \langle\mathcal{W}\rangle_{T, V, N} .
$$

In the following, we derive this Widom insertion formula eq. (3.56). When one molecule is inserted into the system box, which is composed of $N$ molecules at the position $\left\{\boldsymbol{r}^{N}\right\}=\left(\boldsymbol{r}_{1}^{1}, \ldots, \boldsymbol{r}_{M}^{N}\right)$, the probability that this inserted molecule is arranged 
on the position $\left\{\boldsymbol{r}^{N+1}\right\}=\left(\boldsymbol{r}_{1}^{N+1}, \ldots, \boldsymbol{r}_{M}^{N+1}\right)$ is (see eq. (3.38)):

$$
P_{\left\{\boldsymbol{r}^{N+1}\right\},\left\{\boldsymbol{r}^{N}\right\}}=\frac{1}{V} d V \sum_{\{\boldsymbol{B}\} \in\left\{\boldsymbol{B}^{\Gamma}\right\}}\left[\prod_{l=2}^{M}\left\{\frac{\exp \left[-\beta u_{l}^{n, n b}\left(\boldsymbol{b}_{\Gamma}^{l}\right)\right]}{w^{n, n b}(l)} k \prod_{i=1}^{k} \frac{\exp \left[-\beta u_{l}^{n, i n t}\left(\boldsymbol{b}_{i}^{l}\right)\right] d V}{C_{l}}\right\}\right] .
$$

With use of this probability and eq. (3.32), the Rosenbluth weight of this inserted molecule is calculated.

$$
\begin{gathered}
\langle\boldsymbol{W}\rangle_{\left\{\boldsymbol{r}^{N+1}\right\},\left\{\boldsymbol{r}^{N}\right\}} \\
=\frac{1}{V} d V \sum_{\{\boldsymbol{B}\} \in\left\{\boldsymbol{B}^{\Gamma}\right\}}\left[\mathcal{W} \prod_{l=2}^{M}\left\{\frac{\exp \left[-\beta u_{l}^{n, n b}\left(\boldsymbol{b}_{\Gamma}^{l}\right)\right]}{w^{n, n b}(l)} k \prod_{i=1}^{k} \frac{\exp \left[-\beta u_{l}^{n, i n t}\left(\boldsymbol{b}_{i}^{l}\right)\right] d V}{C_{l}}\right\}\right] \\
=\frac{1}{V} d V \sum_{\{\boldsymbol{B}\} \in\left\{\boldsymbol{B}^{\Gamma}\right\}}\left[\exp \left[-\beta u^{n, n b}(1)\right] \prod_{l=2}^{M}\{\right. \\
\left.\left.\quad \exp \left[-\beta u_{l}^{n, n b}\left(\boldsymbol{b}_{\Gamma}^{l}\right)\right] \prod_{i=1}^{k} \frac{\exp \left[-\beta u_{l}^{n, i n t}\left(\boldsymbol{b}_{i}^{l}\right)\right] d V}{C_{l}}\right\}\right]
\end{gathered}
$$

In this equation, the notation of the summation $\sum_{\{\boldsymbol{B}\} \in\left\{\boldsymbol{B}^{\Gamma}\right\}}$ is switched to integral and eq. (3.29) shows the relation:

$$
\begin{aligned}
& \langle\mathcal{W}\rangle_{\left\{\boldsymbol{r}^{N+1}\right\},\left\{\boldsymbol{r}^{N}\right\}} \\
& =\frac{1}{V} d V \exp \left[-\beta u^{n, n b}(1)-\beta \sum_{l=2}^{M} u_{l}^{n, n b}\left(\boldsymbol{b}_{\Gamma}^{l}\right)\right] \\
& \quad \times \prod_{l=2}^{M}\left\{\left(\prod_{i=1}^{k-1} \frac{\int \exp \left[-\beta u_{l}^{n, i n t}\left(\boldsymbol{b}_{i}^{l}\right)\right] d V}{C_{l}}\right) \frac{\exp \left[-\beta u_{l}^{n, \text { int }}\left(\boldsymbol{b}_{\Gamma}^{l}\right)\right] d V}{C_{l}}\right\} \\
& =\frac{(d V)^{M} \exp \left[-\beta U_{\left\{\boldsymbol{r}^{N}\right\}}\left(\left\{\boldsymbol{r}^{N+1}\right\}\right)\right]}{\Lambda^{3 M} Z_{1}^{\text {ideal }}(T, V)},
\end{aligned}
$$

where

$$
U_{\left\{\boldsymbol{r}^{N}\right\}}\left(\left\{\boldsymbol{r}^{N+1}\right\}\right) \text { is the total potential energy of the inserted molecule. }
$$

Integral of $\langle\mathcal{W}\rangle_{\left\{\boldsymbol{r}^{N+1}\right\},\left\{\boldsymbol{r}^{N}\right\}}$ with $\left\{\boldsymbol{r}^{N+1}\right\}$ over the whole system yields the average Rosenbluth weight in the particle configuration $\left\{\boldsymbol{r}^{N}\right\}$ :

$$
\langle\mathcal{W}\rangle_{\left\{\boldsymbol{r}^{N}\right\}}=\frac{\int(d V)^{M} \exp \left[-\beta U_{\left\{\boldsymbol{r}^{N}\right\}}\left(\left\{\boldsymbol{r}^{N+1}\right\}\right)\right]}{\Lambda^{3 M} Z_{1}^{\text {ideal }}(T, V)}
$$

Canonical ensemble average of the Rosenbluth weight is calculated from $\langle\mathcal{W}\rangle_{\left\{\boldsymbol{r}^{N}\right\}}$, 
i.e.

$$
\begin{aligned}
\langle\mathcal{W}\rangle_{T, V, N} & =\frac{\int d \boldsymbol{r}_{1}^{1} \ldots d \boldsymbol{r}_{M}^{N}\langle\mathcal{W}\rangle_{\left\{\boldsymbol{r}^{N}\right\}} \exp \left[-\beta U\left(\boldsymbol{r}_{1}^{1}, \ldots, \boldsymbol{r}_{M}^{N}\right)\right]}{\Lambda^{3 N M} N ! Z_{N}(T, V)} \\
& =\frac{\int d \boldsymbol{r}_{1}^{1} \ldots d \boldsymbol{r}_{M}^{N+1} \exp \left[-\beta U_{\left\{\boldsymbol{r}^{N}\right\}}\left(\left\{\boldsymbol{r}^{N+1}\right\}\right)\right] \exp \left[-\beta U\left(\boldsymbol{r}_{1}^{1}, \ldots, \boldsymbol{r}_{M}^{N}\right)\right]}{(N+1)^{-1} \Lambda^{3(N+1) M}(N+1) ! Z_{N}(T, V) Z_{1}^{\text {ideal }}(T, V)} \\
& =\frac{(N+1) Z_{N+1}(T, V)}{Z_{N}(T, V) Z_{1}^{\text {ideal }}(T, V)}
\end{aligned}
$$

Equation (3.48) and logarithm of eq. (3.59) show

$$
\beta \mu_{\text {excess }}(T, V, N)=-\log \left(\frac{(N+1) Z_{N+1}(T, V)}{Z_{N}(T, V) Z_{1}^{\text {ideal }}(T, V)}\right)=-\log \langle\mathcal{W}\rangle_{T, V, N} .
$$

This result is consistent with eq. (3.56).

\subsection{2-a Rosenbluth weight corresponding to the reservoir}

Equations (3.17), (3.47), and (3.56) yield the total chemical potential of the system in canonical ensemble.

$$
\beta \mu(T, V, N)=\log \left(\Lambda^{3} \rho\right)-\log j(T)-\log \langle\mathcal{W}\rangle_{T, V, N},
$$

where $\rho=N / V$ denotes the average molecular density in the system. When this system is equilibrated with the reservoir at the same chemical potential, i.e. the system in grand canonical ensemble at the chemical potential $\mu_{\text {res }}$,

$$
\beta \mu(T, V, N)=\mu_{\text {res }}=\log \left(\Lambda^{3} \rho_{\text {res }}\right)-\log j(T),
$$

where $\rho_{\text {res }}$ denotes the molecular density of the reservoir. This relation determines $\rho_{\text {res }}$, i.e. chemical potential of the reservoir, corresponding to the same system in NVT-ensemble:

$$
\rho_{\mathrm{res}}=\frac{\rho}{\langle\mathcal{W}\rangle_{T, V, N}}
$$

\subsubsection{Total chemical potential}

So far, the excess chemical potential $\beta \mu_{\text {excess }}(T, V, N)$ has been discussed. On the other hand, $\Lambda$ and $j(T)$ in total chemical potential of the system, eq. (3.47), have not been calculated in our simulation. Our simulation is, however, independent of these unknown physical quantities.

In our grand canonical ensemble simulation algorithm in section 3.3, both $\Lambda$ and $j(T)$ disappear; the reservoir is uniquely characterized only by the molecular density in the reservoir unless the molecular architecture, i.e. $j(T)$, is changed. In the corresponding canonical ensemble simulation, this reservoir density is determined from the system molecular density and the excess chemical potential, e.g. eq. (3.61), independently of $\Lambda$ and $j(T)$. 
Total chemical potential in canonical ensemble at a state $\left(T, V_{1}, N_{1}\right)$ and a state $\left(T, V_{2}, N_{2}\right)$ is respectively

$$
\begin{aligned}
& \beta \mu\left(T, V_{1}, N_{1}\right)=\log \left(\Lambda^{3} \frac{N_{1}}{V_{1}}\right)-\log j(T)+\beta \mu_{\text {excess }}\left(T, V_{1}, N_{1}\right), \\
& \beta \mu\left(T, V_{2}, N_{2}\right)=\log \left(\Lambda^{3} \frac{N_{2}}{V_{2}}\right)-\log j(T)+\beta \mu_{\text {excess }}\left(T, V_{2}, N_{2}\right) .
\end{aligned}
$$

Each $\beta \mu\left(T, V_{1}, N_{1}\right)$ and $\beta \mu\left(T, V_{2}, N_{2}\right)$ contains $\Lambda$ and $j(T)$ whereas, in the difference between the chemical potential, these unknown parameters vanish,

$$
\begin{aligned}
\beta \mu\left(T, V_{1}, N_{1}\right)- & \beta \mu\left(T, V_{2}, N_{2}\right) \\
& =\log \left(\frac{N_{1} V_{2}}{N_{2} V_{1}}\right)+\beta \mu_{\text {excess }}\left(T, V_{1}, N_{1}\right)-\beta \mu_{\text {excess }}\left(T, V_{2}, N_{2}\right)
\end{aligned}
$$

This relation indicates that the chemical potential at an arbitrary state, e.g. $\left(T, V_{1}, N_{1}\right)$, is represented by the chemical potential difference from reference, e.g. $\beta \mu\left(T, V_{2}, N_{2}\right)$ in this equation, regardless of $\Lambda$ and $j(T)$. 


\section{Chapter 4}

\section{Free energy calculation}

In this chapter, we discuss methods for the free energy calculation of an ordered structure in particle simulation.

In our simulation, physical properties of a system are determined as an ensemble average over equilibrium probability distribution of particle coordinates. The free energy of a system, however, is not calculated as an ensemble average. The free energy is equivalent to the partition function of the system. Calculating the partition function demands a high dimensional integral over the whole phase space, which requires a large quantity of computational cost.

Instead of the partition function, we calculate a derivative of free energy with a control parameter as an ensemble average, e.g. pressure as a mechanical quantity in the canonical ensemble. The integration of the derivative, called thermodynamic integration (TDI), yields the free energy difference between two different states.

However, for example when one state is in a crystal phase and the other in a fluid phase, a first order transition and concomitant hysteresis occur in the middle of the integration path. No reversible integration paths as a function of physical control parameters, e.g. temperature and density, are found between these two states. Since the stalk morphology and the system of the two apposed bilayers, between which the excess free energy of the stalk is defined as the free energy difference, are two distinct states, the same problem as the crystallization occurs in the thermodynamic integration. For the sake of solving this problem, we have to find reference states, whose free energy is known, or integration paths bypassing the first order transition, i.e. reversible paths.

\subsection{Free energy of crystals}

When we calculate the free energy of crystals, we can choose the Einstein solid, in which particles are tethered to crystal lattice points by harmonic springs without any inter-particle potential, as the reference state [34]. The potential energies of the Einstein solid and the original interacting particles are denoted by $U_{R}\left(\boldsymbol{r}_{1}, \ldots, \boldsymbol{r}_{i}, \ldots, \boldsymbol{r}_{N}\right)$ and $U_{I}\left(\boldsymbol{r}_{1}, \ldots, \boldsymbol{r}_{N}\right)$ respectively in $N V T$-ensemble, where 
$\boldsymbol{r}_{i}$ is coordinates of the $i$-th particle. These two systems are combined into one system by a dimensionless paramter $\lambda$,

$$
U_{\lambda}\left(\boldsymbol{r}_{1}, \ldots, \boldsymbol{r}_{N}\right):=\lambda U_{I}\left(\boldsymbol{r}_{1}, \ldots, \boldsymbol{r}_{N}\right)+(1-\lambda) U_{R}\left(\boldsymbol{r}_{1}, \ldots, \boldsymbol{r}_{N}\right) .
$$

At $\lambda=1$, this combined system is consistent with the original interacting system and at $\lambda=0$ the Einstein solid. The derivative of the Helmholtz free energy of this combined system, denoted by $F_{\lambda}(T, V, N)$, is:

$$
\frac{d}{d \lambda} F_{\lambda}(T, V, N)=-k_{B} T \frac{d}{d \lambda} \log Z_{N}^{\lambda}(T, V)=\left\langle\frac{d}{d \lambda} U_{\lambda}\left(\boldsymbol{r}_{1}, \ldots, \boldsymbol{r}_{N}\right)\right\rangle_{T, V, N}^{\lambda},
$$

where $Z_{N}^{\lambda}(T, V)$ denotes the partition function of this system, $\langle\cdots\rangle_{T, V, N}^{\lambda}$ does the ensemble average at $\lambda$, and

$$
\frac{d}{d \lambda} U_{\lambda}\left(\boldsymbol{r}_{1}, \ldots, \boldsymbol{r}_{N}\right)=U_{I}\left(\boldsymbol{r}_{1}, \ldots, \boldsymbol{r}_{N}\right)-U_{R}\left(\boldsymbol{r}_{1}, \ldots, \boldsymbol{r}_{N}\right) .
$$

The integration of this derivative from $\lambda=0$ to $\lambda=1$ yields the free energy difference between the Einstein solid and the crystal,

$$
F_{\lambda=1}(T, V, N)-F_{\lambda=0}(T, V, N)=\int_{0}^{1} d \lambda\left\langle\frac{d}{d \lambda} U_{\lambda}\left(\boldsymbol{r}_{1}, \ldots, \boldsymbol{r}_{N}\right)\right\rangle_{T, V, N}^{\lambda} .
$$

In this integration, the spring potential is gradually switched off from the reference, i.e. Einstein solid, and the particles are changed to the original interacting particles. Due to only a slight change in the particle configuration between the crystals and the Einstein solids, the integration is free from the first order transition.

\subsection{Helmholtz free energy calculation based on an exter- nal field}

We have discussed the free energy calculation in crystal phase using Einstein solid as the reference. On the other hand in fluid phase, molecules are diffusing over the whole system box. In such a case, no suitable reference states can be found.

We assume that our external field arranges the ideal gas molecules into our selfassembled structure. In this method, the ordered structure is kept with the help of this external field during the thermodynamic integration for the sake of preventing the first-order transition of interacting molecules.

\subsubsection{Molecules in the external field}

We discuss the statistics about $N$ molecules of the same species in $N V T$-ensemble. One molecule consists of $M$ atoms; the total number of the atoms in the system 
box $=M N$. The partition function for this system is denoted by $Z_{N}^{\lambda_{1}, \lambda_{E}}(T, V)$ :

$$
\begin{aligned}
& Z_{N}^{\lambda_{1}, \lambda_{E}}(T, V)= \\
& \frac{1}{\Lambda^{3 N M} N !} \int d \boldsymbol{r}_{1}^{1} d \boldsymbol{r}_{2}^{1} \ldots d \boldsymbol{r}_{M}^{1} d \boldsymbol{r}_{1}^{2} \ldots d \boldsymbol{r}_{M}^{N} \exp \left[-\frac{1}{k_{B} T} U_{\lambda_{I}, \lambda_{E}}\left(\boldsymbol{r}_{1}^{1}, \ldots, \boldsymbol{r}_{M}^{N}\right)\right]
\end{aligned}
$$

where $\boldsymbol{r}_{k}^{l}$ represents the position of the $k$-th atom in the $l$-th molecule, $U_{\lambda_{I}, \lambda_{E}}$ denotes the potential energy in the system, and $\Lambda$ thermal de Broglie wave length (see eq. (3.6)). In our system, particles are interacting with each other and the external field. $U_{\lambda_{I}, \lambda_{E}}$ is defined as:

$$
U_{\lambda_{I}, \lambda_{E}}\left(\boldsymbol{r}_{1}^{1}, \ldots, \boldsymbol{r}_{M}^{N}\right)=H_{\text {spring }}+\lambda_{I} H_{\mathrm{int}}+\lambda_{E} H_{\mathrm{ext}},
$$

where $H_{\text {spring }}$ denotes the total intramolecular potential of the system e.g. bonding, torsion, and bending, $H_{\text {int }}$ other total interaction potential energy of the system, and $H_{\text {ext }}$ the total external field energy of the system. $H_{\text {spring }}$ and $H_{\text {int }}$ are equivalent to the internal and the non-bonded potential energies respectively defined in section 3.2. The dimensionless coefficients $\lambda_{I}$ and $\lambda_{E}$ determine the strength of the interaction and the external field respectively. At $\lambda_{I}=1$ and $\lambda_{E}=0$, this system is consistent with our original interacting system and at $\lambda_{I}=\lambda_{E}=0$ the disordered reference system. The partition function of this original interacting system is

$$
\begin{aligned}
Z_{N}^{\text {interact }}(T, V) & :=Z_{N}^{\lambda_{1}=1, \lambda_{E}=0}(T, V) \\
& =\frac{1}{\Lambda^{3 N M} N !} \int d \boldsymbol{r}_{1}^{1} \ldots d \boldsymbol{r}_{M}^{N} \exp \left[-\frac{1}{k_{B} T}\left(H_{\text {spring }}+H_{\text {int }}\right)\right] .
\end{aligned}
$$

The partition function at $\lambda_{I}=0$ and $\lambda_{E}=1$ is

$$
\begin{aligned}
Z_{N}^{\text {field }}(T, V) & :=Z_{N}^{\lambda_{1}=0, \lambda_{E}=1}(T, V) \\
& =\frac{1}{\Lambda^{3 N M} N !} \int d \boldsymbol{r}_{1}^{1} \ldots d \boldsymbol{r}_{M}^{N} \exp \left[-\frac{1}{k_{B} T}\left(H_{\text {spring }}+H_{\text {ext }}\right)\right] .
\end{aligned}
$$

\subsubsection{Thermodynamic integration based on an external field}

In our thermodynamic integration method based on the external field, the free energy difference between the ideal gas as the reference state and the ordered state is calculated along the integration path via two straight branches on $\lambda_{I} \lambda_{E}$-plane:

i) $C_{1}:=$ branch from $\left(\lambda_{I}, \lambda_{E}\right)=(0,0)$ to $(0,1)$

ii) $C_{2}:=$ branch from $\left(\lambda_{I}, \lambda_{E}\right)=(0,1)$ to $(1,0)$.

On $C_{1}, \lambda_{I}=0$ and on $C_{2}, \lambda_{I}=1-\lambda_{E}$ (see also Fig. 4.1). Along the first branch, we gradually increase the external field strength from the reference state. We assume that our external field constrains the ideal gas molecules into our self-assembled 
structure at the end of this branch. From this end point, the strength of the external field is gradually decreased and the strength of the inter-particle potential is gradually raised. This is the second branch. At the end of this second branch, the external field is completely switched off and the system is composed of interacting molecules as is aimed. The external field is, in general, determined via successive approximation, which is discussed in section 4.2.4.

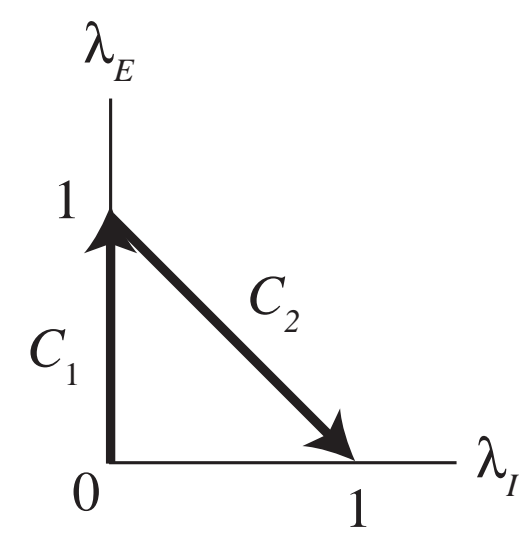

Figure 4.1: Triangular thermodynamic integration path.

On the first branch, our system consists of the ideal gas in the external field. On the second branch, the system keeps the self-assembled structure with the help of the external field. Therefore, we can avoid the first-order transition and calculate the free energy difference between the disordered and ordered states along this triangular integration path. The absence of the first order transition is quantitatively confirmed by the expanded ensemble simulation, which is discussed in section 4.2.3, on each branch.

On this triangular integration path, the derivative of the Helmholtz free energy $F_{\lambda_{E}}(T, V, N)$ with $\lambda_{E}$ is calculated from eqs. (4.1) and (4.2):

$$
\begin{aligned}
& \frac{d}{d \lambda_{E}} F_{\lambda_{E}}(T, V, N) \\
& =-k_{B} T \frac{1}{Z_{N}^{\lambda_{1}, \lambda_{E}}(T, V)} \frac{d Z_{N}^{\lambda_{1}, \lambda_{E}}(T, V)}{d \lambda_{E}} \\
& =\frac{1}{Z_{N}^{\lambda_{1}, \lambda_{E}}(T, V)} \frac{1}{\Lambda^{3 N M} N !} \int d \boldsymbol{r}_{1}^{1} \ldots d \boldsymbol{r}_{M}^{N} \exp \left[-\frac{1}{k_{B} T} U_{\lambda_{I}, \lambda_{E}}\left(\boldsymbol{r}_{1}^{1}, \ldots, \boldsymbol{r}_{M}^{N}\right)\right] \\
& \times \begin{cases}H_{\mathrm{ext}} & \left(\mathrm{On} C_{1}\right) \\
H_{\mathrm{ext}}-H_{\mathrm{int}} & \left(\mathrm{On} C_{2}\right)\end{cases} \\
& =\left\{\begin{array}{ll}
\left\langle H_{\mathrm{ext}}\right\rangle_{T, V, N}^{\lambda_{I}=0, \lambda_{E}} & \left(\text { On } C_{1}\right) \\
\left\langle H_{\mathrm{ext}}-H_{\mathrm{int}}\right\rangle_{T, V, N}^{\lambda_{1}, \lambda_{E}} & \left(\text { On } C_{2}\right)
\end{array},\right.
\end{aligned}
$$


where $\langle\cdots\rangle_{T, V, N}^{\lambda_{1}, \lambda_{E}}$ denotes the ensemble average on a point $\left(\lambda_{I}, \lambda_{E}\right)$. Threfore, the Helmholtz free energy difference between $(0,0)$ and $(1,0)$, denoted by $\Delta F_{C_{1}+C_{2}}$, is:

$$
\Delta F_{C_{1}+C_{2}}=\int_{C_{1}}\left\langle H_{\mathrm{ext}}\right\rangle_{T, V, N}^{\lambda_{I}=0, \lambda_{E}} d \lambda_{E}+\int_{C_{2}}\left\langle H_{\mathrm{ext}}-H_{\mathrm{int}}\right\rangle_{T, V, N}^{\lambda_{1}, \lambda_{E}} d \lambda_{E}
$$

where each of the Helmholtz free energy differences on $C_{1}$ and $C_{2}$ are:

$$
\begin{gathered}
\int_{C_{1}}\left\langle H_{\mathrm{ext}}\right\rangle_{T, V, N}^{\lambda_{I}=0, \lambda_{E}} d \lambda_{E}=\int_{0}^{1}\left\langle H_{\mathrm{ext}}\right\rangle_{T, V, N}^{\lambda_{I}=0, \lambda_{E}} d \lambda_{E} \\
\int_{C_{2}}\left\langle H_{\mathrm{ext}}-H_{\mathrm{int}}\right\rangle_{T, V, N}^{\lambda_{1}, \lambda_{E}} d \lambda_{E}=\int_{0}^{1}\left(\left\langle H_{\mathrm{int}}\right\rangle_{T, V, N}^{\lambda_{I}, \lambda_{E}}-\left\langle H_{\mathrm{ext}}\right\rangle_{T, V, N}^{\lambda_{1}, \lambda_{E}}\right) d \lambda_{E}
\end{gathered}
$$

\subsubsection{Method of expanded ensembles}

In thermodynamic integration eq. (4.6), the integrand over the interval $0 \leq \lambda_{E} \leq 1$ is independently calculated on each discrete points along the branches $C_{1}$ and $C_{2}$ in molecular simulation.

In method of expanded ensembles [60], the ensemble is expanded into $\left(m_{1}+1\right)$ subensembles, i.e.:

$$
0=\lambda_{E}^{(0)}<\lambda_{E}^{(1)}<\cdots<\lambda_{E}^{\left(m_{1}\right)}=1 .
$$

The partition function of this expanded ensemble is defined as:

$$
Z=\sum_{m=0}^{m_{1}} Z_{N}^{(m)}(T, V) \exp \left[\eta_{m}\right]
$$

where $\eta_{m}=$ const. and

$$
\begin{aligned}
Z_{N}^{(m)}(T, V) & :=\frac{1}{\Lambda^{3 N M} N !} \int d \boldsymbol{r}_{1}^{1} \ldots d \boldsymbol{r}_{M}^{N} \exp \left[-\frac{1}{k_{B} T} U^{(m)}\left(\boldsymbol{r}_{1}^{1}, \ldots, \boldsymbol{r}_{M}^{N}\right)\right] \\
U^{(m)}\left(\boldsymbol{r}_{1}^{1}, \ldots, \boldsymbol{r}_{M}^{N}\right) & =H_{\text {spring }}+\lambda_{I}^{(m)} H_{\mathrm{int}}+\lambda_{E}^{(m)} H_{\mathrm{ext}} .
\end{aligned}
$$

On the branch $C_{1}, \lambda_{I}^{(m)}=0$ and on $C_{2}, \lambda_{I}^{(m)}=1-\lambda_{E}^{(m)}$. In this simulation method, each subensemble is successively simulated in one simulation run on each branch.

First we discuss the method of expanded ensembles along the branch $C_{1}$. On this straight branch,

$$
U^{(m)}\left(\boldsymbol{r}_{1}^{1}, \ldots, \boldsymbol{r}_{M}^{N}\right)=H_{\text {spring }}+\lambda_{E}^{(m)} H_{\text {ext }} .
$$

Monte Carlo simulation via expanded ensembles is performed based on the algorithm:

1) Usual displacement via Metropolis algorithm in the fixed $\lambda_{E}^{(k)}$ during a constant time period i.e. constant MCS. 
2) Another subensemble $\lambda_{E}^{(m)}, m=k \pm 1$, is chosen at random. The current subensemble $\lambda_{E}^{(k)}$ is changed to $\lambda_{E}^{(m)}$ with the fixed particle configuration via the acceptance criterion

$$
w_{k \rightarrow m}:= \begin{cases}\min \left(1, \exp \left[\beta\left(\lambda_{E}^{(k)}-\lambda_{E}^{(m)}\right) H_{\mathrm{ext}}+\eta_{m}-\eta_{k}\right]\right), & 0 \leq m \leq m_{1} \\ 0, & m=-1, m_{1}+1\end{cases}
$$

3) Repeat steps 1) and 2).

In this simulation via the method of the expanded ensembles, $p_{m}:=$ the probability that the system is found in the subensemble $\lambda_{E}^{(m)}$ is measured:

$$
p_{m}=\frac{n_{m}}{n_{\text {total }}}
$$

where $n_{\text {total }}$ denotes the total length of the MC chain and $n_{m}$ the number of MCS in the subensemble $\lambda_{E}^{(m)}$. On the other hand, with use of the partition function,

$$
p_{m}=\frac{Z_{N}^{(m)}(T, V) \exp \left[\eta_{m}\right]}{Z}
$$

and therefore

$$
\begin{aligned}
\frac{p_{m}}{p_{k}} & =\frac{Z_{N}^{(m)}(T, V)}{Z_{N}^{(k)}(T, V)} \exp \left[\eta_{m}-\eta_{k}\right] \\
& =\exp \left[-\beta F_{m}+\beta F_{k}+\eta_{m}-\eta_{k}\right],
\end{aligned}
$$

where $F_{m}:=-k_{B} T \log Z_{N}^{(m)}(T, V)$ is Helmholtz free energy of the subensemble $\lambda_{E}^{(m)}$. Thus, we can know the difference of Helmholtz free energy between any pairs of the subensembles using the relations (4.9) and (4.10).

On the branch $C_{2}$,

$$
U^{(m)}\left(\boldsymbol{r}_{1}^{1}, \ldots, \boldsymbol{r}_{M}^{N}\right)=H_{\text {spring }}+H_{\mathrm{int}}+\lambda_{E}^{(m)}\left(H_{\mathrm{ext}}-H_{\mathrm{int}}\right) .
$$

The acceptance criterion eq. (4.8) is:

$$
w_{k \rightarrow m}:= \begin{cases}\min \left(1, \exp \left[\beta\left(\lambda_{E}^{(k)}-\lambda_{E}^{(m)}\right)\left(H_{\mathrm{ext}}-H_{\mathrm{int}}\right)+\eta_{m}-\eta_{k}\right]\right), & 0 \leq m \leq m_{1} \\ 0, & m=-1, m_{1}+1\end{cases}
$$

Optimal $\eta_{m}$ In theory, the simulation results should not be dependent on the choice of the constants $\eta_{m}$. In practice, however, the probability $p_{m}$ strongly depend on $\eta_{m}$ (e.g. if all $\eta_{m}=0$, the system is mostly found in the subensemble of the lowest energy). At $\eta_{m}=\beta F_{m}$, the system is homogeneously distributed over all the subensembles, i.e. $p_{m}=$ const. $=1 /\left(m_{1}+1\right)$. Since this optimal $\eta_{m}$ is determined from unknown physical quantity before simulation, i.e. free energy $F_{m}, \eta_{m}$ is estimated via preliminary simulation runs, e.g. thermodynamic integration (see section 4.2.2). 


\subsubsection{Determining the external field}

The external field which arranges the ideal gas into and mimics the ordered structure observed in the self-assembling system, in other words the external field that distributes the ideal gas into the molecular density distribution calculated in the self-assembling system, is essential for our free energy calculation technique. The absence of the abrupt structural changes on the branch $C_{2}$ indicates that this branch bypasses the first order transition, i.e. free from the hysteresis. In this section, we discuss the external field calculation for the solvent-free linear amphiphiles introduced in section 2.2. We assume that the energy from the external field is,

$$
\frac{H_{\mathrm{ext}}}{k_{B} T}=\int_{V} \frac{d V}{R_{e}^{3}}\left(\rho_{p \mathrm{~A}}^{\prime}(\boldsymbol{r}) W_{\mathrm{A}}(\boldsymbol{r})+\rho_{p \mathrm{~B}}^{\prime}(\boldsymbol{r}) W_{\mathrm{B}}(\boldsymbol{r})\right),
$$

where the dimensionless molecular density has been defined in eq. (2.19) and $W_{\eta}(\boldsymbol{r})$ denotes the external field acting on $\eta$-species of segments at the position $\boldsymbol{r}$.

In systems of dense polymer solutions, self-consistent field theory yields an accurate estimation for the external ordering field [36,37],

$$
W_{\eta}(\boldsymbol{r})=\left.R_{e}^{3} \frac{\delta H_{\mathrm{int}}}{\delta \rho_{p \eta}^{\prime}(\boldsymbol{r})}\right|_{\rho_{p \eta}^{\prime}(\boldsymbol{r})=\tilde{\rho}_{p \eta}^{\prime}(\boldsymbol{r})},
$$

where $\tilde{\rho}_{p \eta}^{\prime}(\boldsymbol{r})$ is the molecular density distribution of $\eta$-species in the self-assembling system i.e. the target structure, which can be calculated prior to this external field calculation.

In the solvent-free systems, however, fluctuations cannot be neglected. Therefore, we propose successive approximation. In this method, we iteratively improve the external fields from the initial estimate via eq. (4.13).

1) Set the initial external fields estimated via eq. (4.13).

2) In these external fields, simulate ideal molecules according to the Hamiltonian eq. (4.12) and calculate the molecular density distribution in the system.

3) Using this result, improve the estimate for the external fields via

$$
W_{\eta}^{(n+1)}(\boldsymbol{r})=W_{\eta}^{(n)}(\boldsymbol{r})+\epsilon\left\{\rho_{p \eta,(n)}^{\prime}(\boldsymbol{r})-\tilde{\rho}_{p \eta}^{\prime}(\boldsymbol{r})\right\},
$$

where $n$ is the iteration index, $\epsilon$ a small positive constant parameter, and $\rho_{p \eta,(n)}^{\prime}(\boldsymbol{r})$ is the molecular density distribution obtained in step 2).

4) Repeat steps 2) and 3) till the convergence.

\subsection{Grand potential calculation}

We have discussed the Helmholtz free energy calculation. Now grand potential, i.e. free energy in $\mu V T$-ensemble, calculation is discussed. Grand potential is defined as

$$
\Phi_{G}(\mu, T, V):=-k_{B} T \log \Xi(\mu, T, V)
$$


where $\Xi(\mu, T, V)$ denotes the grand partition function,

$$
\Xi(\mu, T, V):=\sum_{N=0}^{\infty}\left\{\exp \left[\frac{\mu}{k_{B} T} N\right] Z_{N}(T, V)\right\} .
$$

In thermodynamics,

$$
\Phi_{G}(\mu, T, V)=F\left(T, V,\langle N\rangle_{\mu, T, V}\right)-\mu\langle N\rangle_{\mu, T, V},
$$

where $\langle\cdots\rangle_{\mu, T, V}$ denotes the ensemble average at $\mu, T$, and $V$. Using thermodynamic integration, we determine the difference in the grand potential between the systems at $\mu=\mu^{(1)}$ and $\mu=\mu^{(0)}$.

The derivative of the grand potential eq. (4.15) is:

$$
\begin{aligned}
\frac{\partial}{\partial \mu} \Phi_{G}(\mu, T, V) & =-\frac{1}{\Xi(\mu, T, V)} \sum_{N=0}^{\infty}\left\{N \exp \left[\frac{\mu}{k_{B} T} N\right] Z_{N}(T, V)\right\} \\
& =-\langle N\rangle_{\mu, T, V}
\end{aligned}
$$

Integration of this derivative yields the grand potential difference between $\mu^{(1)}$ and $\mu^{(0)}$ :

$$
\begin{aligned}
\Delta \Phi_{G}^{\mu^{(1)}, \mu^{(0)}} & :=\Phi_{G}\left(\mu^{(1)}, T, V\right)-\Phi_{G}\left(\mu^{(0)}, T, V\right)=\int_{\mu^{(0)}}^{\mu^{(1)}}\left\{\frac{\partial}{\partial \mu} \Phi_{G}(\mu, T, V)\right\} d \mu \\
& =\int_{\mu^{(0)}}^{\mu^{(1)}}\left(-\langle N\rangle_{\mu, T, V}\right) d \mu .
\end{aligned}
$$

During this thermodynamic integration in $\mu V T$-ensemble, the first-order transition can occur unless the states between $\left(\mu^{(1)}, T, V\right)$ and $\left(\mu^{(0)}, T, V\right)$ are in the same phase. To bypath this transition, in theory, the thermodynamic integration based on the external field can also be applied to $\mu V T$-ensemble. In practice, however, the external fields used in $\mu V T$-ensemble could have to be fine tuned for the purpose of keeping the number of molecules approximately a constant. Although such highly adjusted external fields are determined, linear dependence of $\lambda_{E}$ on $\lambda_{I}$ along the branches, i.e. the triangular integration path in Fig. 4.1, would not be sufficient to bypath the first-order transition.

Instead of this method based on the external fields in $\mu V T$-ensemble, we present another integration path bypassing the transition, i.e. the thermodynamic integration in $\mu V T$-ensemble via $N V T$-ensemble:

1) A state in the canonical ensemble $\left(T, V, N_{\text {canonical }}\right)$, which is in the same phase as the state $\left(\mu^{(1)}, T, V\right)$ in the grand canonical ensemble, is set.

2) The Helmholtz free energy at this state, $F\left(T, V, N_{\text {canonical }}\right)$, is calculated along the path that bypasses the first-order transition. This has been discussed in this chapter. The chemical potential in the canonical ensemble, $\mu\left(T, V, N_{\text {canonical }}\right)$, is also determined (see section 3.4). We assume the condition $\mu\left(T, V, N_{\text {canonical }}\right) \approx$ $\mu^{(1)}$; no first-order transition occurs in the region between $\mu\left(T, V, N_{\text {canonical }}\right)$ and $\mu^{(1)}$ with the fixed $T$ and $V$. 
3) The grand potential at the state in the grand canonical ensemble corresponding to the state $\left(T, V, N_{\text {canonical }}\right)$ is, by eq. (4.16),

$$
\Phi_{G}\left(\mu\left(T, V, N_{\text {canonical }}\right), T, V\right)=F\left(T, V, N_{\text {canonical }}\right)-\mu\left(T, V, N_{\text {canonical }}\right) N_{\text {canonical }} .
$$

Substituting $\mu\left(T, V, N_{\text {canonical }}\right)$ for $\mu^{(0)}$ in eq. (4.18) and using eq. (4.19), we obtain the grand potential at $\left(\mu^{(1)}, T, V\right)$,

$$
\begin{aligned}
\Phi_{G}\left(\mu^{(1)}, T, V\right)= & F\left(T, V, N_{\text {canonical }}\right)-\mu\left(T, V, N_{\text {canonical }}\right) N_{\text {canonical }} \\
& +\int_{\mu\left(T, V, N_{\text {canonical }}\right)}^{\mu^{(1)}}\left(-\langle N\rangle_{\mu, T, V}\right) d \mu
\end{aligned}
$$

Due to the conditions in steps 1) and 2), the first-order transition is bypassed through the total integration path. Therefore, the grand potential at arbitrary $\mu^{(1)}$ is calculated via this equation as long as these conditions are satisfied.

This method means that the thermodynamic integration path in the canonical ensemble, e.g. the triangular integration path in Fig. 4.1, is extended to the state in the grand canonical ensemble, $\left(\mu^{(1)}, T, V\right)$.

\subsection{Semigrand canonical ensemble}

We have discussed the free energy calculation methods of single component systems. When we calculate the free energy difference between single component systems composed of distinct molecular species, the same free energy calculation scheme, which takes vast amounts of computation, has to be repeated for each molecular species in order to compare the free energies.

Here, we introduce the semigrand canonical ensemble [61-63] which alters one molecular species to another species. This ensemble yields a direct thermodynamic integration path connecting these two single component systems. Along this path, the Helmholtz free energy difference beteween these systems is directly determined.

\subsubsection{Statistics of semigrand canonical ensemble}

The statistics in the canonical ensemble and the grand canonical ensemble have been discussed. In the grand canonical ensemble the chemical potential, $\mu$, of the system is fixed though the number of molecules, $N$, fluctuates around the average that corresponds to $\mu$. In a binary systems composed of linear amphiphiles, $\alpha$ and $\gamma$-species, that have 2 different block ratios, the grand partition function (see also 
eq. (3.25)) is defined as:

$$
\begin{aligned}
\Xi\left(\mu_{\alpha}, \mu_{\gamma}, T, V\right):= & \sum_{N_{\alpha}=0}^{\infty} \sum_{N_{\gamma}=0}^{\infty}\left\{\frac{\exp \left[\left(\mu_{\alpha} / k_{B} T\right) N_{\alpha}\right]}{\Lambda^{3 N_{\alpha} M} N_{\alpha} !} \frac{\exp \left[\left(\mu_{\gamma} / k_{B} T\right) N_{\gamma}\right]}{\Lambda^{3 N_{\gamma} M} N_{\gamma} !}\right. \\
& \left.\times \int d \boldsymbol{r}_{1}^{1} \ldots d \boldsymbol{r}_{M}^{N_{\alpha}+N_{\gamma}} \exp \left[-\frac{1}{k_{B} T} U\left(\boldsymbol{r}_{1}^{1}, \ldots, \boldsymbol{r}_{M}^{N_{\alpha}+N_{\gamma}}\right)\right]\right\}
\end{aligned}
$$

where $\mu_{\alpha}, \mu_{\gamma}:=$ chemical potential of $\alpha, \gamma$-species, and $N_{\alpha}, N_{\gamma}:=$ the number of molecules in the system of $\alpha, \gamma$-species, respectively. Under the condition that the total number of the molecules in the system box, $N:=N_{\alpha}+N_{\gamma}$, is fixed, this partition function (4.21) is:

$$
\begin{aligned}
\Xi_{N}\left(\mu_{\alpha}, \mu_{\gamma}, T, V\right): & =\exp \left[\frac{\mu_{\gamma}}{k_{B} T} N\right] \frac{1}{\Lambda^{3 N M}} \sum_{N_{\alpha}=0}^{N}\left\{\frac{1}{N_{\alpha} !\left(N-N_{\alpha}\right) !} \exp \left[\frac{\mu_{\alpha}-\mu_{\gamma}}{k_{B} T} N_{\alpha}\right]\right. \\
& \left.\times \int d \boldsymbol{r}_{1}^{1} \ldots d \boldsymbol{r}_{M}^{N_{\alpha}+N_{\gamma}} \exp \left[-\frac{1}{k_{B} T} U\left(\boldsymbol{r}_{1}^{1}, \ldots, \boldsymbol{r}_{M}^{N_{\alpha}+N_{\gamma}}\right)\right]\right\} .
\end{aligned}
$$

This is called semigrand canonical ensemble. In this ensemble, the molecules moves between the reservoir and the system under the constraint that $N$ is fixed. The probability density of $N_{\alpha}$, corresponding to eq. (3.27), is obtained from (4.22),

$$
\begin{aligned}
H_{\mu_{\alpha}, \mu_{\gamma}, T, V, N}\left(N_{\alpha}\right):= & \\
\frac{1}{\Xi_{N}\left(\mu_{\alpha}, \mu_{\gamma}, T, V\right)} & \exp \left[\frac{\mu_{\gamma}}{k_{B} T} N\right] \frac{1}{\Lambda^{3 N M}} \frac{1}{N_{\alpha} !\left(N-N_{\alpha}\right) !} \exp \left[\frac{\mu_{\alpha}-\mu_{\gamma}}{k_{B} T} N_{\alpha}\right] \\
& \times \int d \boldsymbol{r}_{1}^{1} \ldots d \boldsymbol{r}_{M}^{N_{\alpha}+N_{\gamma}} \exp \left[-\frac{1}{k_{B} T} U\left(\boldsymbol{r}_{1}^{1}, \ldots, \boldsymbol{r}_{M}^{N_{\alpha}+N_{\gamma}}\right)\right] .
\end{aligned}
$$

This semigrand canonical ensemble is also reproduced in $N V T$-ensemble; $N$ molecules confined within the system box switch their species according to the partition function of semigrand canonical ensemble [50]. This means that each term in eq. (4.22) corresponds to the combination that $N_{\alpha}$ of $N$ molecules are chosen as $\alpha$-species, i.e. ${ }_{N} C_{N_{\alpha}}=N ! /\left(N_{\alpha} !\left(N-N_{\alpha}\right)\right.$ !). In this $N V T$-ensemble, the summation in eq. (4.22) is replaced with the summation $\sum_{\text {comb. }}$ which runs over all the combinations that $N$ molecules are divided into $\alpha$ and $\gamma$.

$$
\begin{aligned}
\Xi_{N}\left(\mu_{\alpha}, \mu_{\gamma}, T, V\right)= & \frac{1}{N !} \exp \left[\frac{\mu_{\gamma}}{k_{B} T} N\right] \frac{1}{\Lambda^{3 N M}} \sum_{\text {comb. }}\left\{\exp \left[\frac{\mu_{\alpha}-\mu_{\gamma}}{k_{B} T} N_{\alpha}\right]\right. \\
& \left.\times \int d \boldsymbol{r}_{1}^{1} \ldots d \boldsymbol{r}_{M}^{N_{\alpha}+N_{\gamma}} \exp \left[-\frac{1}{k_{B} T} U\left(\boldsymbol{r}_{1}^{1}, \ldots, \boldsymbol{r}_{M}^{N_{\alpha}+N_{\gamma}}\right)\right]\right\} .
\end{aligned}
$$

In this partition function of the semigrand canonical ensemble, each term has been divided by ${ }_{N} C_{N_{\alpha}}$ for the purpose of correcting the double counts. 
The partition function (4.22) shows the relation

$$
N_{\alpha} \approx\left\{\begin{array}{ll}
N, & \mu_{\alpha} \gg \mu_{\gamma} \\
0, & \mu_{\alpha} \ll \mu_{\gamma}
\end{array} .\right.
$$

In this limit,

$$
\Xi_{N}\left(\mu_{\alpha}, \mu_{\gamma}, T, V\right)= \begin{cases}\exp \left[\frac{\mu_{\alpha}}{k_{k} T} N\right] Z_{N}^{\alpha}(T, V), & \mu_{\alpha} \gg \mu_{\gamma} \\ \exp \left[\frac{\mu_{\gamma}}{k_{B} T} N\right] Z_{N}^{\gamma}(T, V), & \mu_{\alpha} \ll \mu_{\gamma}\end{cases}
$$

where $Z_{N}^{a}(T, V):=$ partition function of $a$-species single component system in canonical ensemble (see eq. (3.6)).

\subsubsection{Semigrand canonical ensemble simulation}

Systems in the semigrand canonical ensemble is simulated based on the partition function (4.24) by the following methods:

1) In one simulation step, with constant probability $p_{s}$, trial species change is chosen.

i) One molecule of $N$ is selected at random. $U_{\text {old }}:=$ the energy of this molecule in the current state is calculated.

ii) The species of the selected molecule is changed. $U_{\text {new }}:=$ the energy of this molecule after this change is calculated. This trial species change is accepted according to the acceptance criterion,

$$
\operatorname{acc}(a \rightarrow b)=\min \left(1, \exp \left[-\frac{1}{k_{B} T}\left(\mu_{a}-\mu_{b}+U_{\text {new }}-U_{\text {old }}\right)\right]\right),
$$

where $a, b:=$ old and new molecular species respectively. If rejected, the molecular species is returned to the state before this trial.

2) In one simulation step, with probability $1-p_{s}$, a local trial movement via Metropolis algorithm is chosen.

The acceptance criterion (4.26) indicates that the chemical potetial difference $\mu_{\alpha}-$ $\mu_{\gamma}$ determines simulation results, regardless of each chemical potential $\mu_{\alpha}$ and $\mu_{\gamma}$.

\subsubsection{Free energy difference via semigrand canonical simulation}

Using semigrand canonical ensemble, we calculate the Helmholtz free energy difference between single component systems composed of distinct molecular species.

First, a physical quantity corresponding to free energy of semigrand canonical ensemble is defined as

$$
\Psi_{N}\left(\mu_{\alpha}, \mu_{\gamma}, T, V\right):=-k_{B} T \log \Xi_{N}\left(\mu_{\alpha}, \mu_{\gamma}, T, V\right) .
$$


Derivative of this quantity with $\mu_{\alpha}$ is:

$$
\frac{\partial \Psi_{N}\left(\mu_{\alpha}, \mu_{\gamma}, T, V\right)}{\partial \mu_{\alpha}}=-k_{B} T \frac{1}{\Xi_{N}\left(\mu_{\alpha}, \mu_{\gamma}, T, V\right)} \frac{\partial \Xi_{N}\left(\mu_{\alpha}, \mu_{\gamma}, T, V\right)}{\partial \mu_{\alpha}}=-\left\langle N_{\alpha}\right\rangle_{T, \mu_{\alpha}, \mu_{\gamma}, V, N}
$$

where $\langle\cdots\rangle_{T, \mu_{\alpha}, \mu_{\gamma}, V, N}:=$ semigrand canonical ensemble average. These equations yield the integration relation,

$$
\begin{aligned}
\Delta \Psi_{N, \mu_{\gamma}, T, V} & \left(\mu_{\alpha}^{(1)}, \mu_{\alpha}^{(0)}\right) \\
& :=\Psi_{N}\left(\mu_{\alpha}^{(1)}, \mu_{\gamma}, T, V\right)-\Psi_{N}\left(\mu_{\alpha}^{(0)}, \mu_{\gamma}, T, V\right) \\
& =\int_{\mu_{\alpha}^{(0)}}^{\mu_{\alpha}^{(1)}} \frac{\partial \Psi_{N}\left(\mu_{\alpha}, \mu_{\gamma}, T, V\right)}{\partial \mu_{\alpha}} d \mu_{\alpha}=\int_{\mu_{\alpha}^{(0)}}^{\mu_{\alpha}^{(1)}}\left(-\left\langle N_{\alpha}\right\rangle_{T, \mu_{\alpha}, \mu_{\gamma}, V, N}\right) d \mu_{\alpha} .
\end{aligned}
$$

At $\mu_{\alpha}^{(1)} \gg \mu_{\gamma}$ and $\mu_{\alpha}^{(0)} \ll \mu_{\gamma}$, eqs. (4.25), (4.27), and (4.29) show

$$
\Delta \Psi_{N, \mu_{\gamma}, T, V}\left(\mu_{\alpha}^{(1)}, \mu_{\alpha}^{(0)}\right)=F_{\alpha}(T, V, N)-F_{\gamma}(T, V, N)-N\left(\mu_{\alpha}^{(1)}-\mu_{\gamma}\right),
$$

where $F_{\alpha}(T, V, N)$ and $F_{\gamma}(T, V, N):=$ Helmholtz free energy of single component systems in canonical ensemble composed of $\alpha$ and $\gamma$-species respectively. Therefore, from eqs. (4.30) and (4.31), we obtain the Helmholtz free energy difference between the single component systems

$$
\begin{aligned}
\Delta F_{\gamma, \alpha}(T, V, N): & =F_{\gamma}(T, V, N)-F_{\alpha}(T, V, N) \\
& =\int_{\mu_{\alpha}^{(0)}}^{\mu_{\alpha}^{(1)}}\left(\left\langle N_{\alpha}\right\rangle_{T, \mu_{\alpha}, \mu_{\gamma}, V, N}\right) d \mu_{\alpha}-N\left(\mu_{\alpha}^{(1)}-\mu_{\gamma}\right) .
\end{aligned}
$$

This result connects a molecular species $\alpha$ with another species $\gamma$ via the direct thermodynamic integration path, sketched in Fig 4.2. During this integration, the single component system of one species is mutated to the other species.

\subsubsection{Test simulation in semigrand canonical ensemble}

As a test, we simulate a binary mixture of linear amphiphiles with the number of segments per molecule $=32$ using our solvent-free model discussed in section 2.2. The $\alpha$-species is defined as an amphiphile composed of 28 A-segments and $\gamma$ species 27 A-segments. Single component systems of $\alpha$-species are simulated in chapter 5 . In this section, $n_{p}$ denotes the total number of amphiphiles fixed at 932, $n_{p \alpha}$ denotes the number of $\alpha$-species, and $n_{p \gamma}$ does $\gamma$-species. In the initial state, $n_{p \alpha}=932$ amphiphilic molecules are arranged on the bilayer configuration parallel to $y z$-plane in the system box $L_{x} / R_{e} \times L_{y} / R_{e} \times L_{z} / R_{e}=10 \times 4 \times 4$, which corresponds to a tensionless bilayer of $\alpha$-species. The width of the cell, $\Delta L$, and the maximum trial displacement are given in Table. 5.1. In this test simulation, $10 \%$ of the total simulation steps are taken as the trial molecular species change on average.

Figure 4.3 shows the $\left(n_{p \alpha} / n_{p}\right)$ vs chemical potential difference graph. In the region $\mu_{\alpha}-\mu_{\gamma} \gg 0, n_{p \alpha} / n_{p}$ is approaching to 1 and in $\mu_{\alpha}-\mu_{\gamma} \ll 0$ to 0 . The 


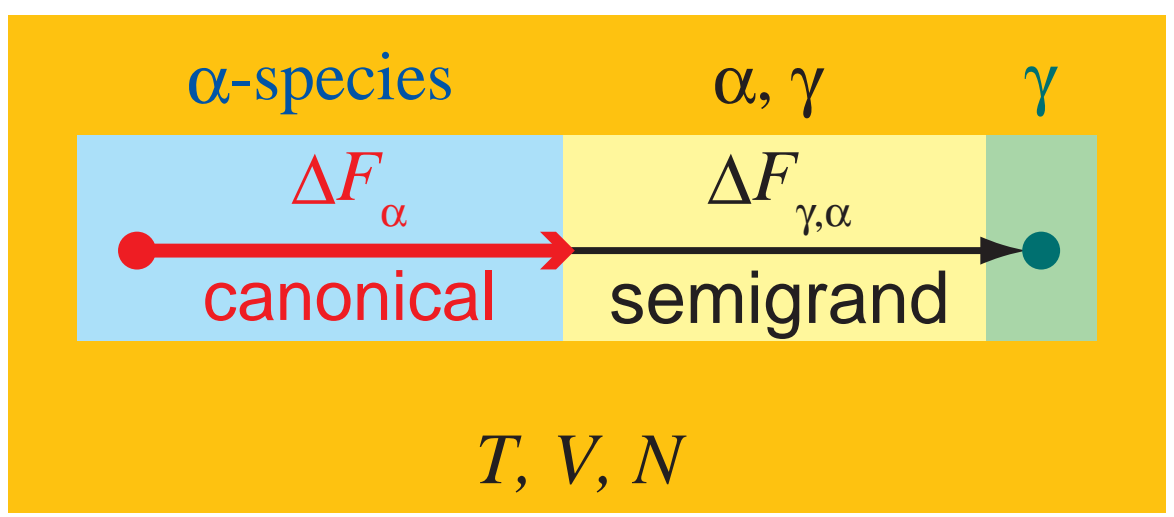

Figure 4.2: Thermodynamic integration paths via semigrand canonical ensemble. $\Delta F_{\alpha}$ denotes the free energy difference between the reference state and the single component system of $\alpha$-species. From this system, the molecules are mutated to another species, $\gamma$. Free energy difference between the reference and the single component system composed of $\gamma$-species is equal to $\Delta F_{\alpha}+\Delta F_{\gamma, \alpha}$. On these integration paths, $T, V$, and $N$ are fixed.

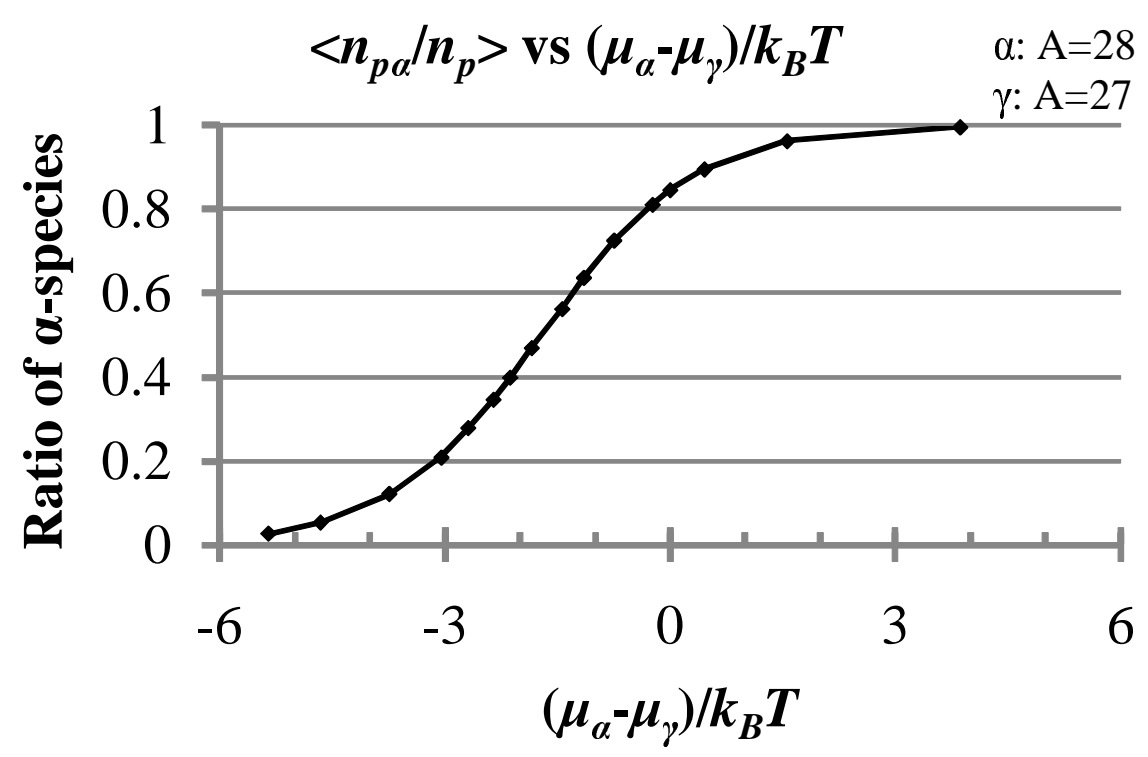

Figure 4.3: $\left(n_{p \alpha} / n_{p}\right)$ vs chemical potential difference between $\alpha$ and $\gamma$. Data are sampled every MCS from $500 \mathrm{MCS}$, the systems are equilibrated by this MCS, to 1,000 MCS and 501 independent samples are obtained. This continuous line shows the absence of the $1 \mathrm{st}$ order transition in this region; the free energy difference is calculated from this graph via eq. (4.32).

continuous line of this graph indicates that no first order transition occurs. In these simulations, the system keeps the bilayer structure (see Fig. 4.4).

Figure 4.5 shows probability densities of $n_{p \alpha}$ at $\Delta \mu:=\mu_{\alpha}-\mu_{\gamma}=0.3053 k_{B} T$ and 


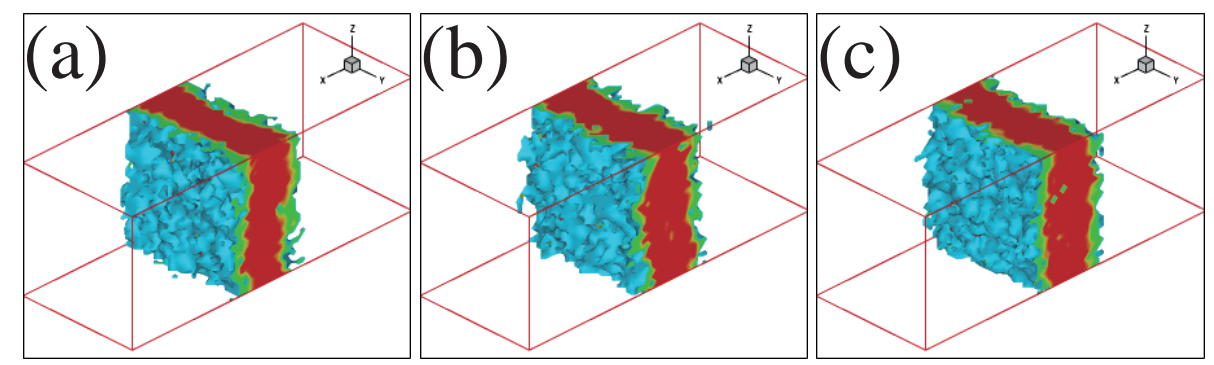

Figure 4.4: Snapshots of the system at $7 \times 10^{5}$ MCS. Red regions in these snapshots represent the regions in which A-block of the amphiphiles gather and green regions do B-block. (a): taken at $\left(\mu_{\alpha}-\mu_{\gamma}\right) / k_{B} T=-4.656$, (b): -1.843 , and (c): 0.4595 . The system keeps the bilayer structure in our simulation.

$0.4595 k_{B} T$. In the inset, the left-hand side of the relation obtained from eq. (4.23),

$$
\begin{array}{r}
\frac{H_{\mu_{\alpha}^{\prime}, \mu_{\gamma}^{\prime}, T, V, N}\left(N_{\alpha}\right)}{H_{\mu_{\alpha}, \mu_{\gamma}, T, V, N}\left(N_{\alpha}\right)} \exp \left[-\frac{\left(\mu_{\alpha}^{\prime}-\mu_{\gamma}^{\prime}\right)-\left(\mu_{\alpha}-\mu_{\gamma}\right)}{k_{B} T} N_{\alpha}\right] \\
=\frac{\Xi_{N}\left(\mu_{\alpha}, \mu_{\gamma}, T, V\right)}{\Xi_{N}\left(\mu_{\alpha}^{\prime}, \mu_{\gamma}^{\prime}, T, V\right)} \exp \left[\frac{\mu_{\gamma}^{\prime}-\mu_{\gamma}}{k_{B} T} N\right]=: C_{\text {semi }},
\end{array}
$$

is plotted as a function of $n_{p \alpha}$, where $\mu_{\alpha}^{\prime}-\mu_{\gamma}^{\prime}=0.4595 k_{B} T$ and $\mu_{\alpha}-\mu_{\gamma}=0.3053 k_{B} T$ in our simulation. This constant graph is consistent with this relation, $C_{\text {semi }}$.

These test simulation results show the validity of our simulation. 


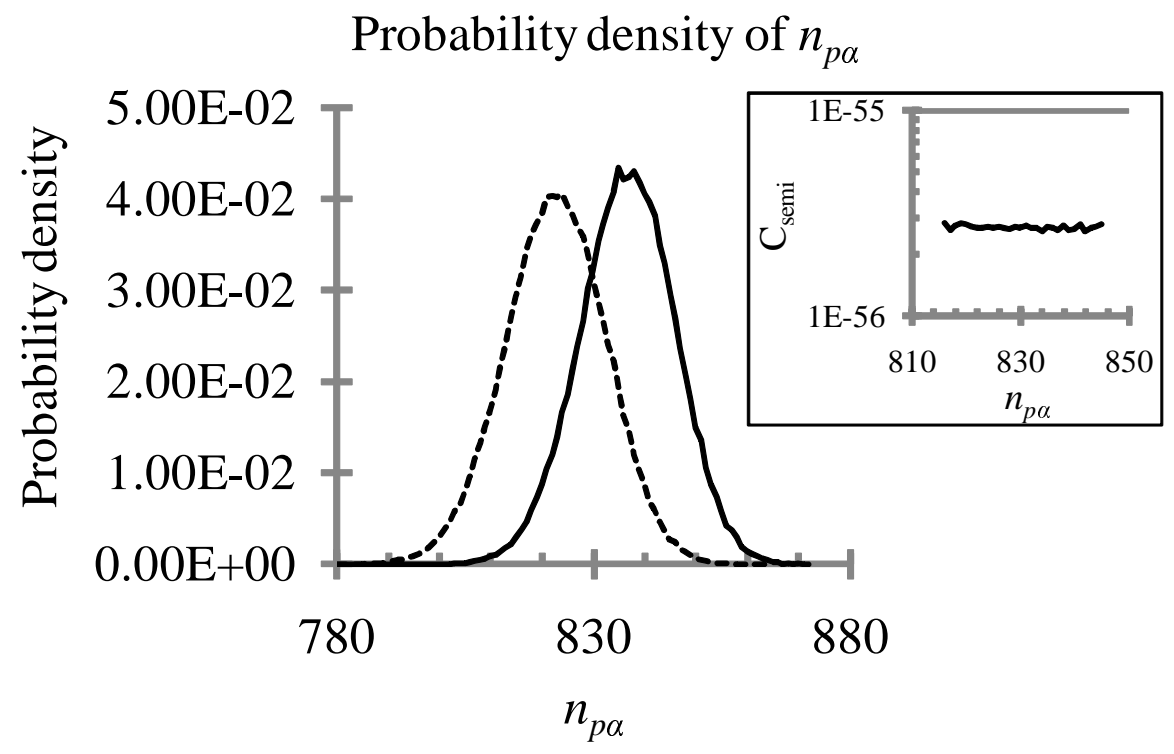

Figure 4.5: Probability densities of $n_{p \alpha}$ at $\Delta \mu:=\mu_{\alpha}-\mu_{\gamma}=0.3053 k_{B} T$ and $0.4595 k_{B} T$ plotted by a broken line and a solid line respectively. Data are sampled every 5 MCS after 500 MCS and 139,901 independent samples are obtained. $C_{\text {semi }}$ as a function of $n_{p \alpha}$ is plotted in the inset. 


\section{Chapter 5}

\section{Simulation of bilayer membranes}

Using the solvent-free model of linear amphiphiles, i.e. lipids, discussed in section 2.2, we simulate bilayer membranes coupled with our improved PPPM method (see section 2.3). We measure the excess free energy of the stalk in the systems composed of molecules with $N_{A}$, the number of A-segments in one lipid, $=27$, 28 , and 29 where the number of interaction centers per molecule equals 32 . The free energy of the systems at $N_{A}=28$ is first measured. Based on this result, the free energy at $N_{A}=27$ and 29 is also determined via the semigrand canonical ensemble. These simulations show the dependence of the stalk stability on molecular architecture, i.e. the block-ratio of the molecules. We also calculate the tension of the bilayer membranes, which allows us to plot the free energy of the stalk as a function of the tension.

Fixed parameters in these simulations are given in Table 5.1. Thermal energy $k_{B} T$ is taken as the energy unit.

First, the single-component isolated tensionless bilayers composed of lipids with $N_{A}=27,28$ and 29 are simulated as the reference systems.

Table 5.1: Fixed simulation parameters for the solvent-free lipid model.

\begin{aligned} & The number of interaction centers per molecule $N=32 \\ &$ The width of the cell $\Delta L=(1 / 6) R_{e} \\ &$ The maximum trial displacement $=\Delta L \\ &$\hline\end{aligned}

\subsection{Simulation results of tensionless bilayers}

In this section, we simulate the single component isolated tensionless bilayer at $N_{A}=28$ and 29 in the canonical ensemble. 


\subsubsection{Tensionless bilayers at $N_{A}=28$}

The tensionless bilayers consisting of lipids with $N_{A}=28$ in the canonical ensemble are discussed. For the purpose of measuring the membrane thickness at the tensionless state, i.e. the tension of membranes $\sigma=0$, we study the bilayer in the configuration where the bilayers arranged parallel to the $y z$-plane span the periodic system box only in the $z$-direction, as is shown in the lower inset of Fig. 5.1. In this system, two free edges are formed in the $y$-direction. Therefore in the canonical ensemble, the bilayer shrinks or grows in the $y$-direction by itself until reaching the tensionless state. This bilayer patch composed of $n_{p}=1280$ molecules is simulated in the system box, $L_{x} / R_{e} \times L_{y} / R_{e} \times L_{z} / R_{e}=8.0 \times 20.0 \times 2.0$, which is large enough to simulate an isolated membrane. Obtained molecular density profiles across the membrane in the $x$-direction, i.e. the normal direction to the membrane, at this tensionless state are shown in the main plate of Fig. 5.1. The bilayer thickness of tail regions, $2 d_{c}$, is estimated from this density profile, i.e. $2 d_{c}=1.3 R_{e}$. Comparing this thickness with typical experimental results [64], $2 d_{c} \approx 30(\AA)$, the length scale in our simulation is obtained, $R_{e}=23.0(\AA)$. The integration of the density profiles yield the area per amphiphilic molecule, $A_{0}=0.0343 R_{e}^{2}=18.1\left(\AA^{2}\right)$. The peak of the hydrophobic tails in the density profile, approximately 40 , is consistent with the result of the mean field approximation, eq. (2.22).

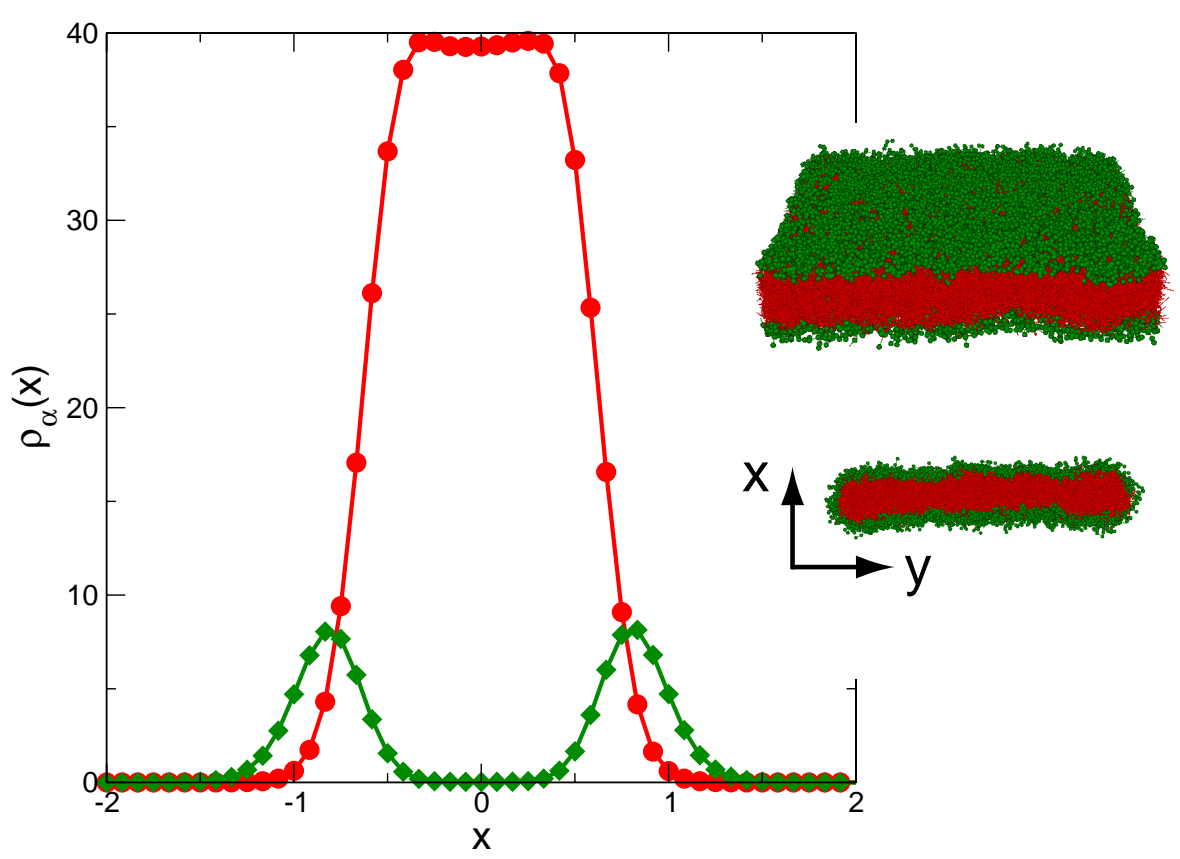

Figure 5.1: Molecular density profile across the tensionless bilayer with the free edges. The lower inset shows a snapshot of an isolated tensionless bilayer with the two free edges. The upper inset is a snapshot of the tensionless bilayer consisting of 5830 molecules which span the system box both in $y$-direction and $z$-direction. The red and the green in these pictures show the hydrophobic tails and the hydrophilic heads respectively. 
Using this $A_{0}$ calculated in our simulation, we can pre-assemble a tensionless bilayer parallel with the $y z$-plane that spans the system box both in the $y$-direction and in the $z$-direction, as is shown in the upper inset of Fig. 5.1. Figure 5.2 shows the mean square displacement (MSD) of the center of mass for one amphiphile swimming on this tensionless bilayer composed of $2 L_{y} L_{z} / A_{0}=5830$ amphiphiles in the cubic system box $L_{x} / R_{e} \times L_{y} / R_{e} \times L_{z} / R_{e}=10 \times 10 \times 10$. Since the molecules are confined in the bilayer, the lateral diffusion constant of the lipids on the bilayer, denoted by $D$, is determined from the relation,

$$
D \cong \frac{1}{4 t}\left\langle|\boldsymbol{r}(t)-\boldsymbol{r}(0)|^{2}\right\rangle,
$$

where $t$ is time measured in units of MCS in our simulation, and $\boldsymbol{r}(t):=$ the position of the center of mass of a lipid at time $t$. From this equation and linear fitting of MSD shown in Fig. 5.2,

$$
D=2.95 \times 10^{-5}\left(\frac{R_{e}^{2}}{\mathrm{MCS}}\right)
$$

\section{MSD of the center of mass of polymers $N_{\mathrm{A}}=28$ single tensionless}

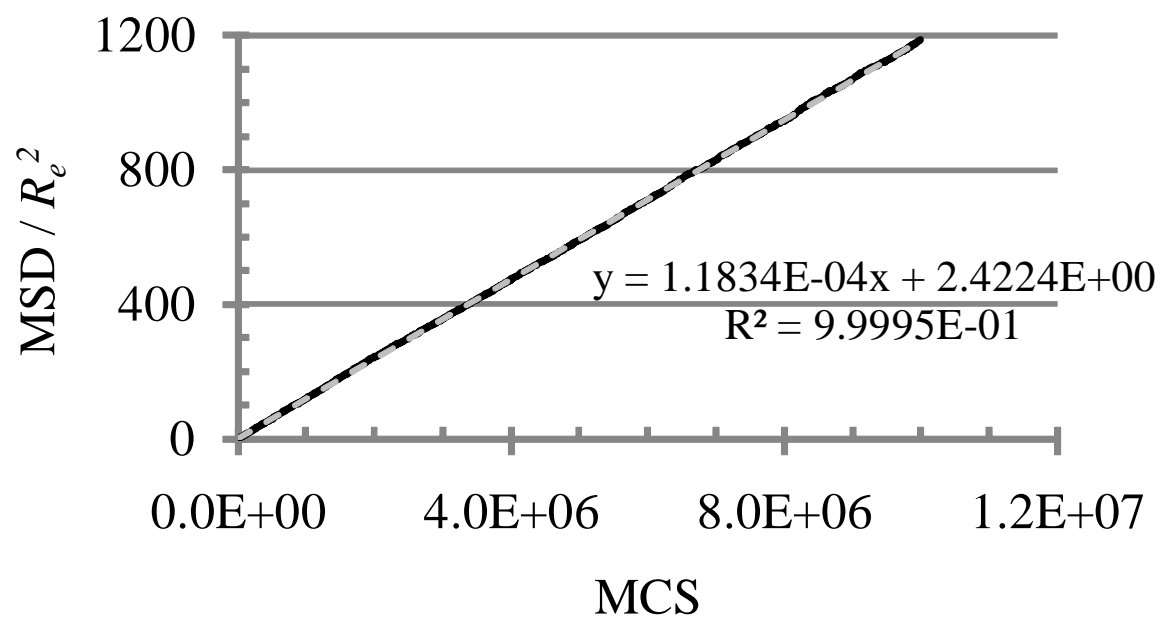

Figure 5.2: Mean square displacement of one molecule at $N_{A}=28$ in the single tensionless bilayer composed of $2 L_{y} L_{z} / A_{0}=5830$ amphiphiles. The system size is $L_{x} / R_{e} \times L_{y} / R_{e} \times$ $L_{z} / R_{e}=10 \times 10 \times 10$. The solid black line and the broken grey line represent MSD and the linear fitting of MSD respectively. This fitting indicates the relation, MSD $\propto 1.1834 \times$ $10^{-4} t$. From this result and eq. (5.1), the lateral diffusion constant, $D$, is determined in eq. (5.2).

In order to calculate the excess chemical potential of this tensionless bilayer, denoted by $\mu_{0}^{\mathrm{ex}}$, we arrange the bilayer parallel with the $y z$-plane which spans the 
system box, $L_{x} / R_{e} \times L_{y} / R_{e} \times L_{z} / R_{e}=5 \times 10 \times 10$, in $y$ and $z$-direction. The number of lipids, denoted by $n_{p}$, is fixed at $2 L_{y} L_{z} / A_{0}=5830$. Using about $2 \times 10^{4}$ particle configuration sets, which are sampled every $1 \times 10^{4}$ MCS, we calculate the excess chemical potential of the system via eq. (3.55), i.e. the particle deletion and the insertion without any bias. Figure 5.3 shows the results, that is, $\mu_{0}^{\mathrm{ex}}=-37.745 k_{B} T$. This corroborates the result via Rosenbluth sampling eq. (3.56), $\mu_{0}^{\mathrm{ex}}=-37.740 k_{B} T$. This excess chemical potential and the average molecular density in the system, $n_{p} / L_{x} L_{y} L_{z}$, yield the total chemical potential, defined in eq. (3.47), of this system.

We measure the excess chemical potential via Rosenbluth sampling.

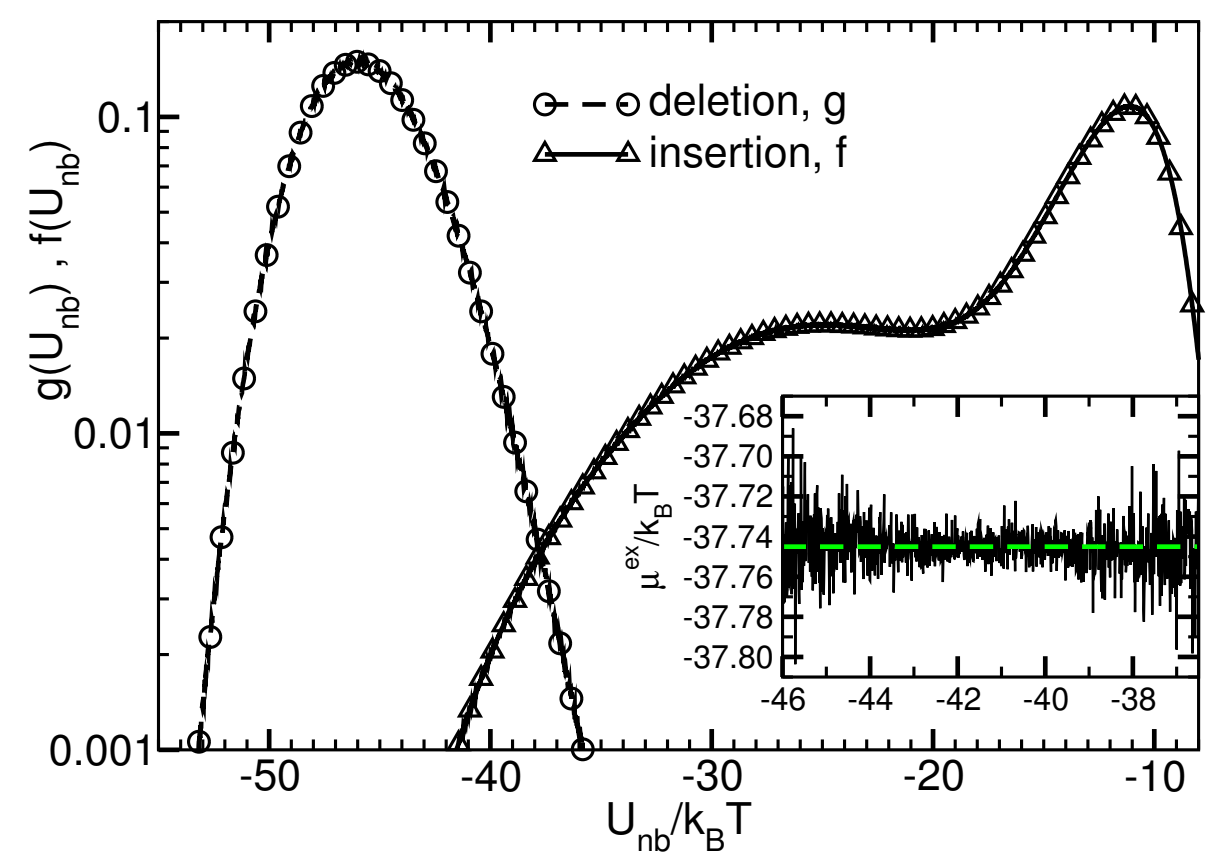

Figure 5.3: Chemical potential calculation of a tensionless bilayer at $N_{A}=28$ via Widom's insertion and deletion method introduced in section 3.4.1. Molecules are deleted and inserted without any bias. Probability distributions of excess non-bonded interaction potential $U_{\mathrm{nb}}$ on the particle deletion and the particle insertion, functions $g$ and $f$ are shown. In the inset, the excess chemical potential is plotted according to eq. (3.55). The dashed line in the inset marks our estimate for the excess chemical potential via this method.

\subsubsection{Tensionless bilayers at $N_{A}=29$ and 27}

At $N_{A}=29, A_{0}=0.03314 R_{e}^{2}$ is obtained. This $A_{0}$ is smaller than the value at $N_{A}=28$ because the hydrophilic heads in amphiphiles are effectively smaller. The excess chemical potential of the tensionless bilayer composed of $2 L_{y} L_{z} / A_{0}=6030$ which spans the system $L_{x} / R_{e} \times L_{y} / R_{e} \times L_{z} / R_{e}=5 \times 10 \times 10$ in $y$ and $z$-direction is $\mu_{0}^{\mathrm{ex}}=-37.740 k_{B} T$. 
At $N_{A}=27, A_{0}=0.0352 R_{e}^{2}$. The excess chemical potential of the tensionless bilayer, $\mu_{0}^{\mathrm{ex}}=-36.044 k_{B} T$, is determined in a simulation box of size $L_{x} / R_{e} \times$ $L_{y} / R_{e} \times L_{z} / R_{e}=5 \times 10 \times 10$, composed of $2 L_{y} L_{z} / A_{0}=5680$ molecules. 


\subsection{Helmholtz free energy of the stalk at $N_{A}=28$}

Using the simulation results of tensionless membranes in section 5.1, we simulate the stalk morphology and the two apposed bilayers composed of lipids with $N_{A}=28$ in order to measure the free energy of the stalk. The Helmholtz free energy of the stalk is measured in the caonical ensemble via the thermodynamic integration and the expanded ensemble simulation (see chapter 4) on the paths sketched in Fig. 5.4. On these paths, the total number of molecules in the system $n_{p}$, the temperature $T$, and the system volume $V$ are all fixed. In this sketch, the states 1 and 5 are the two apposed bilayers and the stalk morphology respectively. From now on, $F_{i}$ denotes the Helmholtz free energy on the state $i, \mu_{i}$ the chemical potential on the state $i$, and $\mu_{i}^{\mathrm{ex}}$ the excess chemical potential on $i$. Each of the paths $3 \rightarrow 4 \rightarrow 5$ and $3 \rightarrow 2 \rightarrow 1$, on which the external fields that reproduce the stalk morphology and the two apposed bilayers are applied respectively, corresponds to the triangle path in Fig. 4.1. The Hamiltonian due to the external field in our solvent-free model has been defined in eq. (4.12).

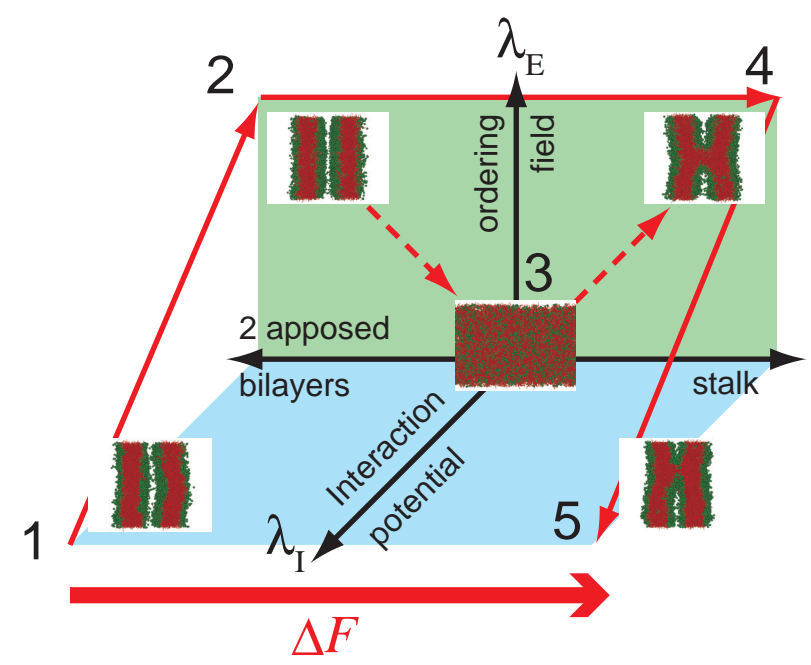

Figure 5.4: Thermodynamic integration paths for the Helmholtz free energy calculation at $N_{A}=28$. Each of the paths $3 \rightarrow 4 \rightarrow 5$ and $3 \rightarrow 2 \rightarrow 1$, on which the external fields that reproduce the stalk morphology and the two apposed bilayers are applied respectively, corresponds to the triangle path in Fig. 4.1. On all the branches in this figure, the total number of molecules in the system $n_{p}$, the temperature $T$, and the system volume $V$ are all fixed.

During the branch $1 \rightarrow 2$, the interaction potential in the system of the two apposed bilayers is gradually replaced with the external ordering fields. These external fields confine the ideal molecules into the two apposed bilayer structure at the end of this branch, state 2 , on which the interaction potential is completely turned off. Along the branch $2 \rightarrow 3$, these fields are gradually switched off and the ideal gas becomes disordered on state 3 . Along the branch $3 \rightarrow 4$, the differ- 
ent external ordering fields is gradually turned on. These different external fields gather the ideal molecules into the stalk morphology on the end of this branch, state 4 . Along the last branch $4 \rightarrow 5$, these external fields are switched off and the interaction potential is gradually turned on. On the last of this path, i.e. state 5, the external fields are completely turned off. Along the total path, we can obtain the Helmholtz free energy difference, i.e. the Helmholtz free energy of the stalk,

$$
\Delta F:=F_{5}-F_{1}=\sum_{i=1}^{4} \Delta F_{i \rightarrow i+1}, \quad \Delta F_{j \rightarrow k}:=F_{k}-F_{j} .
$$

Each of the Helmholtz free energy difference $\Delta F_{j \rightarrow k}$ is given from eq. (4.7):

$$
\begin{aligned}
& \Delta F_{1 \rightarrow 2}=\int_{0}^{1} d \lambda_{E}\left\langle H_{\mathrm{ext}}^{\mathrm{bilayers}}-H_{\mathrm{int}}\right\rangle_{T, V, n_{p}}^{\lambda_{I}=1-\lambda_{E}, \lambda_{E}}, \\
& \Delta F_{2 \rightarrow 3}=\int_{1}^{0} d \lambda_{E}\left\langle H_{\mathrm{ext}}^{\mathrm{bilayers}}\right\rangle_{T, V, n_{p}}^{\lambda_{I}=0, \lambda_{E}}
\end{aligned}
$$

where $H_{\mathrm{ext}}^{\mathrm{stalk}}$ and $H_{\mathrm{ext}}^{\text {bilayers }}$ denote the energy due to the external ordering fields for the stalk morphology and the two apposed bilayers respectively. Similar expressions hold for the branches $4 \rightarrow 5$ and $3 \rightarrow 4$ respectively.

Alternatively, the branches $2 \rightarrow 3 \rightarrow 4$ can be replaced with the direct branch $2 \rightarrow 4$ in order to directly calculate the free energy difference $\Delta F_{2 \rightarrow 4}$. On this direct branch, the external ordering fields for the two apposed bilayers is gradually mutated to the fields for the stalk morphology while the interaction potential energy $H_{\text {int }}$ is always $0 . H_{\text {ext }}$ is defined as the linear superposition of these 2 fields with use of a dimensionless parameter $\lambda$ :

$$
H_{\mathrm{ext}}=\lambda H_{\mathrm{ext}}^{\mathrm{stalk}}+(1-\lambda) H_{\mathrm{ext}}^{\text {bilayers }}, \quad 0 \leq \lambda \leq 1 .
$$

At $\lambda=0$, the system is set on the state 2 and at $\lambda=1$ the state 4 . The Helmholtz free energy difference between the states 2 and 4 via this direct branch is:

$$
\Delta F_{2 \rightarrow 4}=\int_{0}^{1} d \lambda\left\langle H_{\mathrm{ext}}^{\mathrm{stalk}}-H_{\mathrm{ext}}^{\mathrm{bilayers}}\right\rangle_{T, V, n_{p}}^{\lambda}
$$

where the angle brackets represents the canonical ensemble average at the position $\lambda$ on this direct branch.

The relation,

$$
\Delta F_{2 \rightarrow 3}+\Delta F_{3 \rightarrow 4}-\Delta F_{2 \rightarrow 4}=0,
$$

provides a gauge measuring the error in the thermodynamic integration.

The number of the molecules in the system, $n_{p}$, and the fixed system size $\left(L_{x}, L_{y}, L_{z}\right)$ chosen in this Helmholtz free energy calculation is shown in Table 5.2. Hereafter in this thesis, bilayers are assembled parallel to $y z$-plane and span the system box in $y$ and $z$-direction. This means that $x$-direction is normal to bilayers. $n_{p}$ in Table 5.2 is slightly larger than the number of lipids in the two apposed tensionless bilayers that span this system box in $y$ and $z$-direction, i.e. $4 L_{y} L_{z} / A_{0}=4197$.

Hereafter, the superscripts, stalk and bilayers, represent the morphology i.e. the stalk and the two apposed bilayers respectively. 
Table 5.2: System size and the number of lipids $n_{p}$ used for the Helmholtz free energy calculation.

$\begin{aligned} L_{x} & =10.0 R_{e} \\ L_{y} & =6.0 R_{e} \\ L_{z} & =6.0 R_{e} \\ n_{p} & =4240\end{aligned}$

\subsubsection{External field calculation}

We begin with determining the external fields that gather the ideal molecules into the stalk morphology according to the successive approximation discussed in section 4.2.4. For this calculation, $\epsilon=0.05$ is chosen in eq. (4.14). The ideal molecules are simulated in the external fields in the canonical ensemble for 10,000 MCS in order to evaluate the average molecular density distribution. The obtained molecular density distribution is used to improve the external fields and, in the improved external fields, the ideal molecules are simulated again. The convergence was achieved at about 10 iterations.

Figure 5.5 shows 2D contour plots of the molecular density distribution in the self-assembled stalk structure, i.e. the state 5 , and the ideal molecular density distribution structured by the external fields at the final iteration, i.e. the state 4 . These distributions have been radially averaged around the central axis of the stalk. $r$ and $x$ denote the radial distance from the central axis of the stalk and the coordinate normal to the bilayer. This result indicates the similarity between these structures. A similar quality of consistency between the two apposed bilayers of self-assembling and the ideal molecules structured into the bilayers by the improved external fields is also obtained (data not shown).

\subsubsection{Expanded ensemble simulations at $N_{A}=28$}

Using these adjusted external ordering fields, the expanded ensembles simulation introduced in section 4.2.3 is performed along the branches $1 \rightarrow 2,2 \rightarrow 4$, and $4 \rightarrow 5$ which are discretised into $K=58, K=52$, and $K=66$ sampling points respectively. These $K$ ensure that the distribution function at neighbouring sampling points overlap. It is noted that a slightly different $K$ is chosen for the thermodynamic integration.

Figure 5.6 shows the time evolution of $\lambda_{E}$ in the middle of the expanded ensemble simulation on the branches $1 \rightarrow 2$ and $4 \rightarrow 5$. These results indicate that each subensemble is visited with roughly equal probability and that the systems freely switch the subensembles over the whole branch, $0 \leq \lambda_{E} \leq 1$. This means that these branches are free from the first order phase transition, as was aimed. The Helmholtz free energy difference obtained by the expanded ensembles on these three branches is given in Table 5.3. 


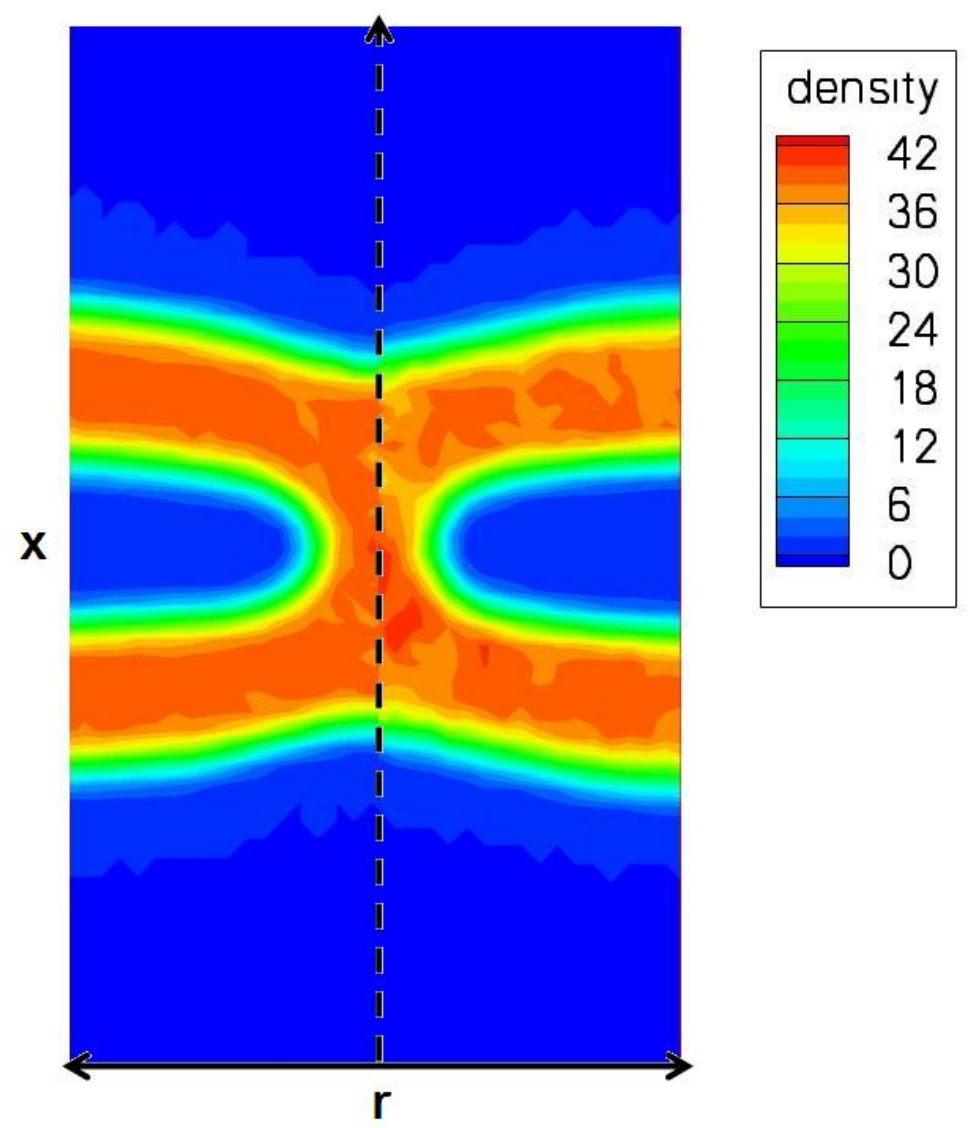

Figure 5.5: 2D contour plot of the molecular distribution for tail blocks, i.e. A-species. The result in self-assembled stalk morphology (left) and the result of ideal gas structured by the external fields determined via the successive approximation (right) are shown. These distributions have been radially averaged around the central axis of the stalk. $r$ and $x$ denote the radial distance from the central axis of the stalk and the coordinate normal to the bilayer respectively.

\subsubsection{Helmholtz free energy difference along each branch}

We show the Helmholtz free energy difference along each branch.

The thermodynamic integrands on each branch are shown in Figs. 5.7, 5.8, and 5.9, where the thermodynamic integrands are plotted in the main panels. The smooth curves in these integrands show the absence of the first order transition, which has been quantitatively confirmed by the expanded ensemble simulation.

Figure 5.7 presents the results for the branches $1 \rightarrow 2$ and $4 \rightarrow 5$. The integrands vary rapidly, approaching the ends of the branches. Around $\lambda_{E}=0$, this behaviour comes from the thermal membrane fluctuations, which occur in the interacting systems i.e. the states 1 and 5. However, this fluctuation is rapidly suppressed by switching on the external fields. The dependence of the integrand on 


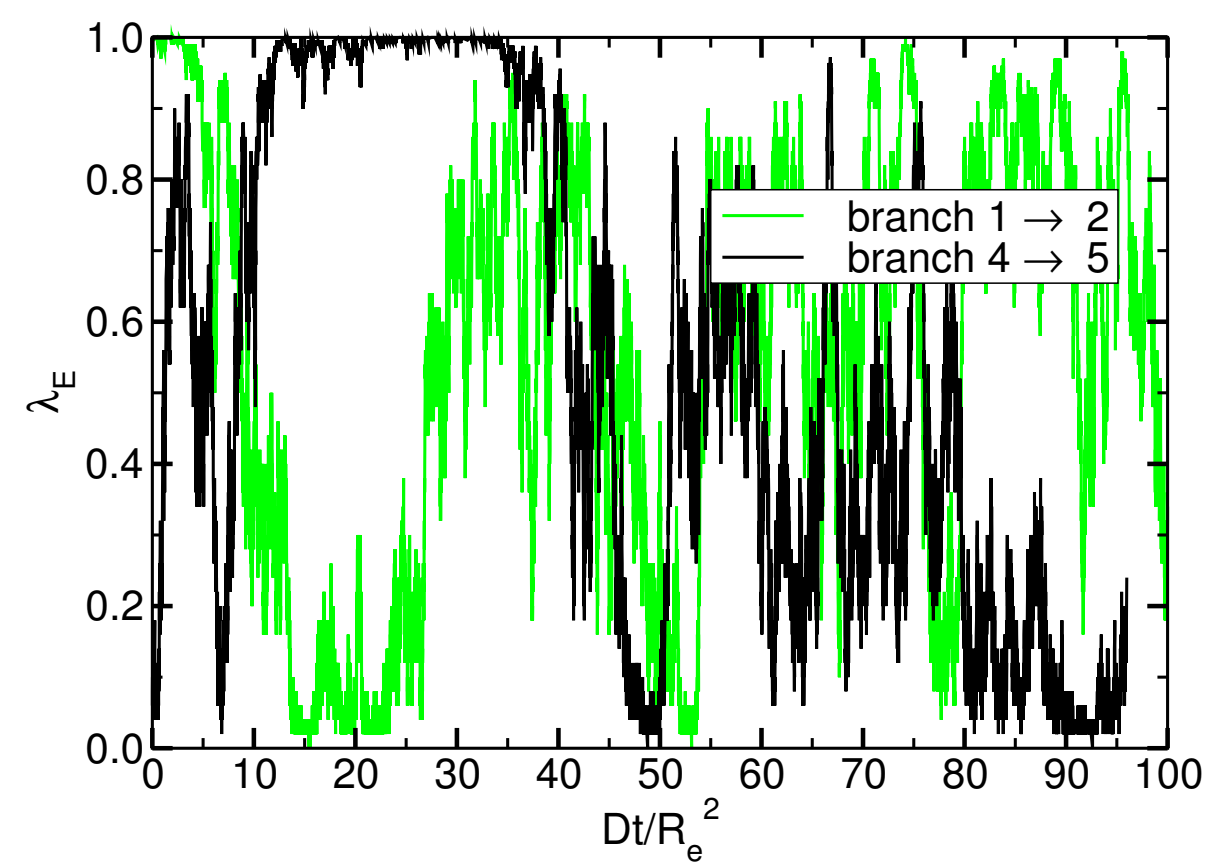

Figure 5.6: Time evolution of $\lambda_{E}$ in the middle of the expanded ensemble simulation on the branches $1 \rightarrow 2$ and $4 \rightarrow 5$ plotted by green and black lines respectively as a function of the lateral motion of one molecule in a tensionless bilayer membrane.

$\lambda_{E}$ at the limit $\lambda_{E} \rightarrow 1$ is rationalized by the strong reduction of compressibility and the concomitant growth of fluctuations as the interparticle interaction potential is completely switched off. The Helmholtz free energy difference is given in Table. 5.3.

Figure 5.8 shows the results for the branches $2 \rightarrow 3$ and $3 \rightarrow 4$, on which the ideal gas gathered into the ordered structure is transformed into a disordered one. In Fig. 5.8, an analytical estimate for the integrands around the disordered state of the ideal gas, the state 3, is also plotted with use of the Random-Phase-Approximation (RPA) [1]. This RPA result corroborates the TDI result at small $\lambda_{E}, \lambda_{E}<0.05$. The dependence of the free energy difference of the stalk and two-bilayer structure on $\lambda_{E}$ is also plotted in the insets of Fig. 5.7 for the branches $1 \rightarrow 2$ and $4 \rightarrow 5$ and Fig. 5.8 for the branches $2 \rightarrow 3$ and $3 \rightarrow 4$.

In Fig. 5.9, the results along the direct branch $2 \rightarrow 4$, where the external field is mutated from the two-bilayer morphology to the stalk morphology, is shown. The free energy along this branch, $\Delta F_{2 \rightarrow 4}(\lambda)$, is plotted in the inset.

From these data, the free energy of the stalk calculated by the TDI is $\Delta F=$ $15.1 k_{B} T$ via the path $1 \rightarrow 2 \rightarrow 3 \rightarrow 4 \rightarrow 5$ and $\Delta F=16.4 k_{B} T$ via the path $1 \rightarrow 2 \rightarrow 4 \rightarrow 5$. These results corroborates the free energy of the stalk accurately determined by the expanded ensemble simulation via the path $1 \rightarrow 2 \rightarrow 4 \rightarrow 5$, $\Delta F=15.3 k_{B} T$ which is used hereafter in this thesis. This positive $\Delta F$ indicates the metastability of the stalk. Our result is consistent with the observation that the 


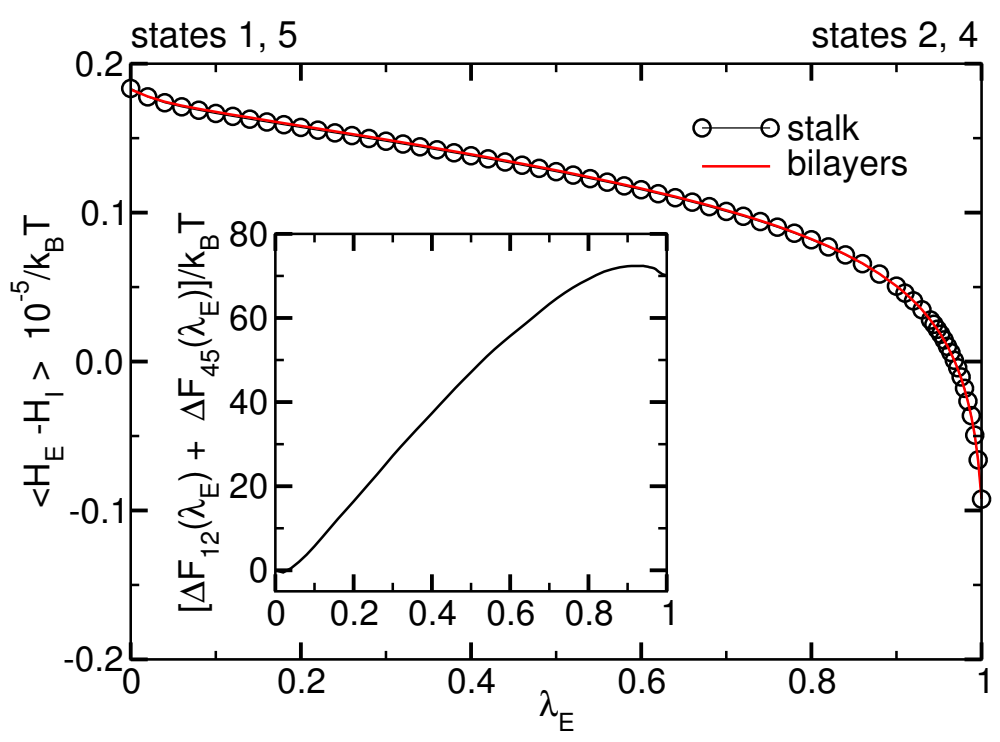

Figure 5.7: The thermodynamic integrands on the branches $1 \rightarrow 2$ and $4 \rightarrow 5$ plotted by red line and black circles respectively. These branches are discretised into 65 sampling points. The inset shows the sum of the free energy changes for the bilayer and stalk structures, obtained after integrating the data of the main figure (with proper sign to account for the direction of the branch), as a function of $\lambda_{E}$.

stalk disappears after long simulation runs.

Table 5.3: The Helmholtz free energy difference on each branch at $N_{A}=28$ via the thermodynamic integration (TDI) and the expanded ensemble simulation (EE). Hereafter in this thesis, the results of the expanded ensemble simulation is used.

\begin{tabular}{rrl}
\hline TDI & $\Delta F_{1 \rightarrow 2}+\Delta F_{4 \rightarrow 5}$ & $=70.108 k_{B} T$ \\
& $\Delta F_{2 \rightarrow 3}+\Delta F_{3 \rightarrow 4}$ & $=-55.038 k_{B} T$ \\
& $\Delta F_{2 \rightarrow 4}$ & $=-53.662 k_{B} T$ \\
\hline EE & $\Delta F_{1 \rightarrow 2}+\Delta F_{4 \rightarrow 5}$ & $=70.813 k_{B} T$ \\
$\Delta F_{2 \rightarrow 4}$ & $=-55.558 k_{B} T$ \\
\hline
\end{tabular}




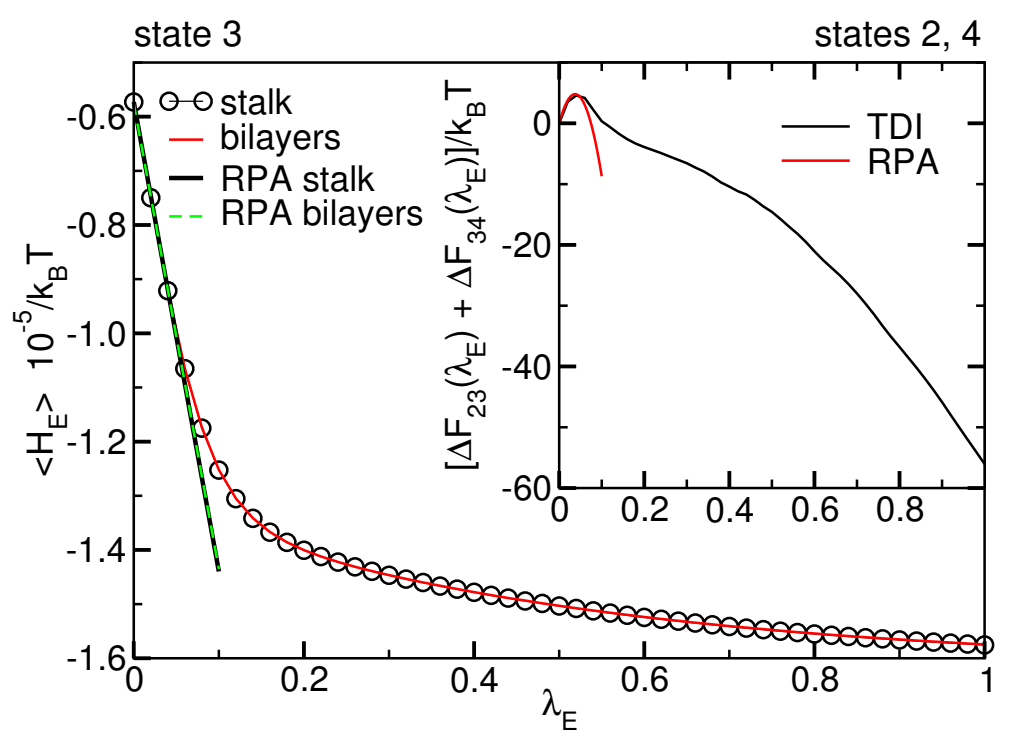

Figure 5.8: The thermodynamic integrands on the branches $2 \rightarrow 3$ and $3 \rightarrow 4$ plotted by red line and black circles respectively. These branches are discretised into 51 sampling points. The inset shows the sum of the free energy changes for the bilayer and stalk structures, obtained after integrating the data of the main figure as a function of $\lambda_{E}$. The prediction of the Random-Phase-Approximation (RPA) [1] for the behaviour of the integrands near the disordered state $\left(\lambda_{E}=0\right)$ are shown with thick solid and dashed lines for the stalk and the two bilayers, respectively. The RPA behaviour of the free energy difference for small values of $\lambda_{E}$ is marked in the inset with red line. This RPA result corroborates the TDI result at small $\lambda_{E}$.

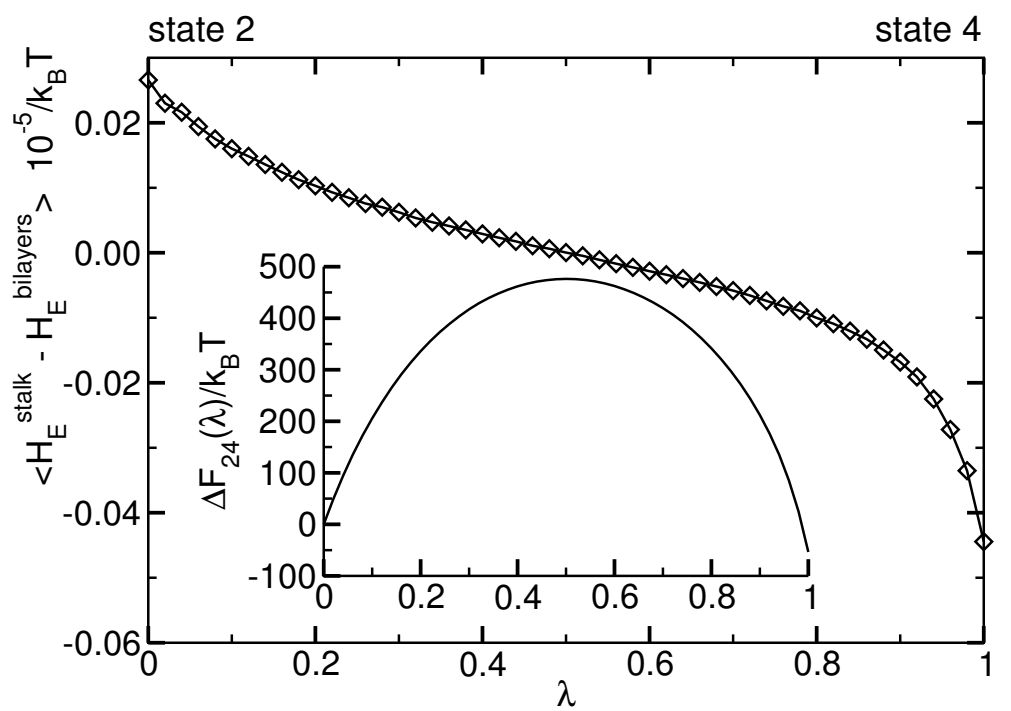

Figure 5.9: The thermodynamic integrand on the branch $2 \rightarrow 4$. This branch is discretised into 51 sampling points. The inset shows the free energy change as a function of $\lambda$ obtained after integrating the curve in the main figure. 


\subsection{Helmholtz free energy of the stalk at $N_{A}=29$}

We measure the excess Helmholtz free energy of the stalk, $\Delta F$, at $N_{A}=29$. This can be calculated by the simulation in the canonical ensemble, the scheme in section 5.2, independently of the simulation at $N_{A}=28$. We choose, however, the semigrand canonical ensemble simulations discussed in section 4.4 instead of these canonical simulations in order to reduce the computation. In our semigrand canonical simulation, $10 \%$ of the total simulation steps are chosen as the trial molecular species change on average.

The integration path based on the semigrand canonical ensemble simulation is sketched in Fig. 5.10. The single component system of the molecular species with $N_{A}=28$ for the stalk morphology is mutated to $N_{A}=29$ from the state 5 via the semigrand canonical ensemble simulation. The apposed bilayers at $N_{A}=29$ is mutated to $N_{A}=28$, i.e. state 1 . The states at $N_{A}=29$ for the apposed bilayers and the stalk morphology linked to $N_{A}=28$ via these semigrand canonical ensembles are denoted by states 6 and 7 respectively. Adding $\Delta F$ at $N_{A}=28$ to TDI on the branches $6 \rightarrow 1$ and $5 \rightarrow 7$, we obtain the Helmholtz free energy of the stalk, $\Delta F=\Delta F_{6 \rightarrow 7}$ at $N_{A}=29$. In these semigrand canonical ensemble simulations, the system size and the total number of molecules are still fixed; the values in Table 5.2 are used.

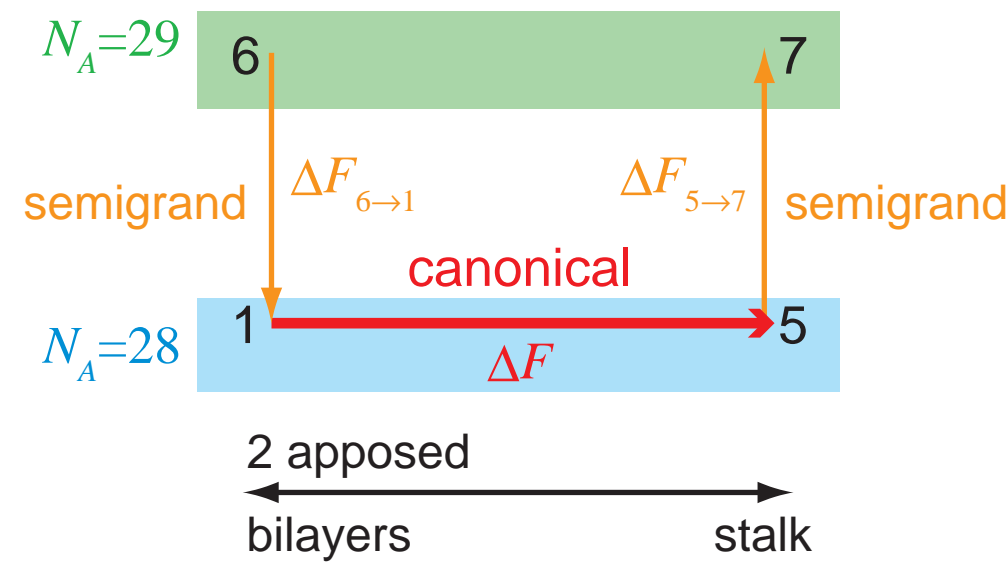

Figure 5.10: Thermodynamic integration paths for the Helmholtz free energy calculation at $N_{A}=29$. The apposed bilayers and the stalk morphology, denoted by 6 and 7 respectively, is connected to the corresponding states at $N_{A}=28$, i.e. the states 1 and 5 respectively, via the semigrand canonical ensemble simulations. Adding $\Delta F$ at $N_{A}=28$ to the thermodynamic integration on the branches $6 \rightarrow 1$ and $5 \rightarrow 7$, we obtain the Helmholtz free energy of the stalk, $\Delta F$ at $N_{A}=29$. Since the integration from the state 1 to the state 5 has been obtained in the simulations at $N_{A}=28$, we can skip the simulations in canonical ensemble at $N_{A}=29$ using these semigrand canonical ensemble simulations.

The semigrand canonical ensemble simulation results, $\left\langle n_{p \alpha}\right\rangle_{T, \mu_{\alpha}, \mu_{\gamma}, V, n_{p}}$, are plotted as a function of the chemical potential difference, $\left(\mu_{\alpha}-\mu_{\gamma}\right) / k_{B} T$, in Fig. 5.11 
where $\alpha$ and $\gamma$-species represent $N_{A}=29$ and $N_{A}=28$ respectively. The smooth form of $\left\langle n_{p \alpha}\right\rangle_{T, \mu_{\alpha}, \mu_{\gamma}, V, n_{p}}$ indicates the absence of the first-order transition. The difference between the data,

$$
\Delta\left\langle n_{p \alpha}\right\rangle_{T, \mu_{\alpha}, \mu_{\gamma}, V, n_{p}}:=\left\langle n_{p \alpha}\right\rangle_{T, \mu_{\alpha}, \mu_{\gamma}, V, n_{p}}^{\text {stalk }}-\left\langle n_{p \alpha}\right\rangle_{T, \mu_{\alpha}, \mu_{\gamma}, V, n_{p}}^{\text {bilayers }},
$$

is presented in the inset, where $\left\langle n_{p \alpha}\right\rangle_{T, \mu_{\alpha}, \mu_{\gamma}, V, n_{p}}^{\text {stalk }}$ and $\left\langle n_{p \alpha}\right\rangle_{T, \mu_{\alpha}, \mu_{\gamma}, V, n_{p}}^{\text {bilayers }}$ denote the results for the stalk morphology and the apposed bilayers respectively. This small difference, $\Delta\left\langle n_{p \alpha}\right\rangle_{T, \mu_{\alpha}, \mu_{\gamma}, V, n_{p}}$, gives the free energy change due to the mutation. From these results and the integration eq. (4.32), the Helmholtz free energy difference is obtained, i.e. $\Delta F_{6 \rightarrow 1}+\Delta F_{5 \rightarrow 7}=-15.9 k_{B} T$. Therefore, the Helmholtz free energy of the stalk is

$$
\left(\Delta F \text { at } N_{A}=29\right)=\Delta F_{6 \rightarrow 1}+\Delta F_{5 \rightarrow 7}+\left(\Delta F \text { at } N_{A}=28\right)=-0.6 k_{B} T \text {. }
$$

This negative $\Delta F$ at $N_{A}=29$ indicates the stability of the stalk; spontaneous stalk formation between the apposed bilayers is also observed in this system. These results show the stability of the stalk at the large molecular assymetry, i.e. large block ratio $N_{A} / N$.

At $\left(\mu_{\alpha}-\mu_{\gamma}\right) / k_{B} T=-2$, where a peak is observed in the inset of Fig. 5.11, the normalized difference of molecular density distribution between both the molecular species,

$$
\frac{\rho_{p \mathrm{~A}(\gamma)}^{\prime}-\rho_{p \mathrm{~A}(\alpha)}^{\prime}}{\rho_{p \mathrm{~A}(\gamma)}^{\prime}+\rho_{p \mathrm{~A}(\alpha)}^{\prime}}
$$

where $\rho_{p \mathrm{~A}(\gamma)}^{\prime}$ denotes the molecular density distribution of hydrophobic tails for $\gamma$ species and $\rho_{p \mathrm{~A}(\alpha)}^{\prime}$ for $\alpha$-species, is measured and plotted in Fig. 5.12. In this graph, negative regions are found on the stalk structure. This result means that $\alpha$-species, more asymmetric molecular architecture $N_{A}=29$, gather around the stalk in the binary mixture simulated in the semigrand canonical ensemble.

\subsection{Helmholtz free energy of the stalk at $N_{A}=27$}

The Helmholtz free energy $\Delta F$ at $N_{A}=27$ is also measured via the semigrand canonical ensemble simulations. The states 1 and 5 are linked to the corresponding states at $N_{A}=27$, the states 8 and 9 respectively, via the semigrand canonical ensemble. The calculated $\left\langle n_{p \alpha}\right\rangle_{T, \mu_{\alpha}, \mu_{\gamma}, V, n_{p}}$ and $\Delta\left\langle n_{p \alpha}\right\rangle_{T, \mu_{\alpha}, \mu_{\gamma}, V, n_{p}}$ are presented in Fig. 5.13. These data yield $\Delta F_{8 \rightarrow 1}+\Delta F_{5 \rightarrow 9}=18.1 k_{B} T$. This indicates that the stalk at $N_{A}=27$ is more unstable than the stalk at $N_{A}=28$ i.e. more asymmetric molecules; this result is consistent with the observation that the stalk disappears in a short simulation runs at $N_{A}=27$. The Helmholtz free energy of the stalk, $\Delta F$ at $N_{A}=27$, is:

$$
\left(\Delta F \text { at } N_{A}=27\right)=\Delta F_{8 \rightarrow 1}+\Delta F_{5 \rightarrow 9}+\left(\Delta F \text { at } N_{A}=28\right)=33.4 k_{B} T .
$$




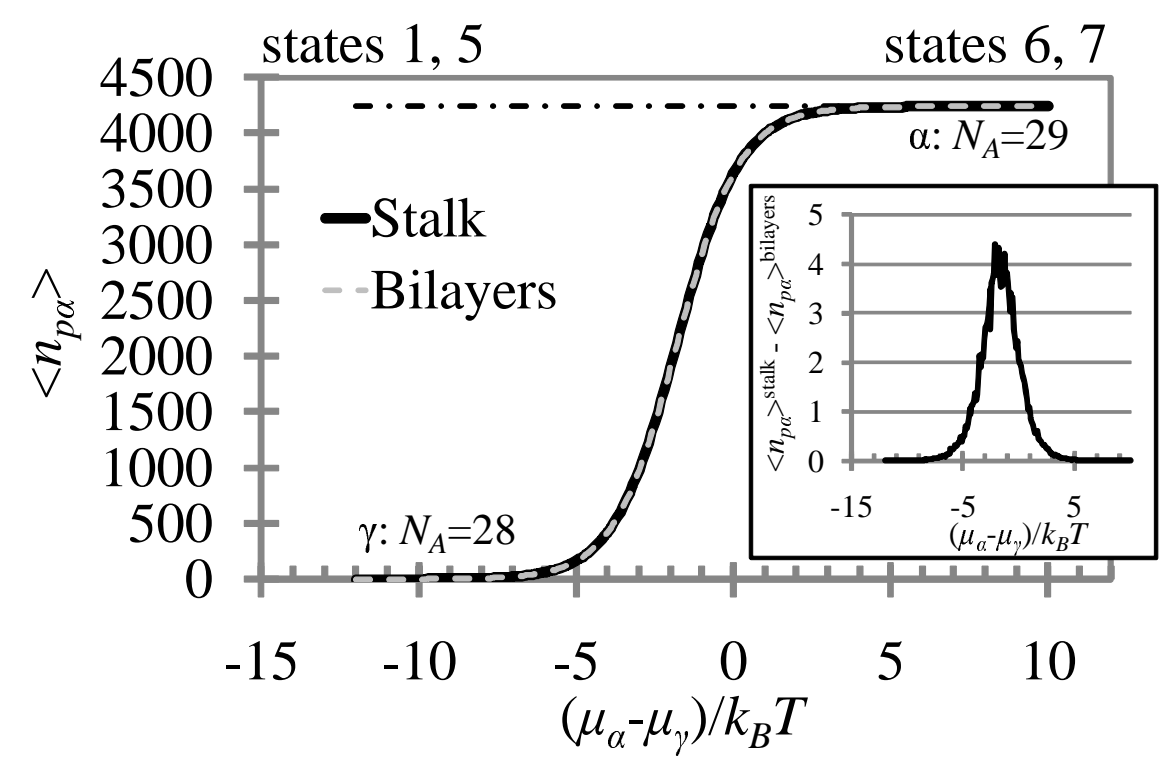

Figure 5.11: Semigrand canonical ensemble simulation results between $N_{A}=29$ and $N_{A}=28$. The main plate shows $\left\langle n_{p \alpha}\right\rangle_{T, \mu_{\alpha}, \mu_{\gamma}, V, n_{p}}$ as a function of the chemical potential difference, $\left(\mu_{\alpha}-\mu_{\gamma}\right) / k_{B} T$, for the stalk morphology and the two apposed bilayers, which are plotted by a black solid line and a broken grey line respectively. Both the branches are set in the interval $-12.0 \leq\left(\mu_{\alpha}-\mu_{\gamma}\right) / k_{B} T \leq 10.0$ and discretised homogeneously into 221 sampling points. $\alpha$-species is the molecules with $N_{A}=29$ and $\gamma$-species $N_{A}=28$. The smooth form of these lines indicates the absence of the first-order transition. The black chain line shows the total number of the molecules, $n_{p}=4240$. The inset presents the difference between the data in the main panel, $\Delta\left\langle n_{p \alpha}\right\rangle_{T, \mu_{\alpha}, \mu_{\gamma}, V, n_{p}}$. 


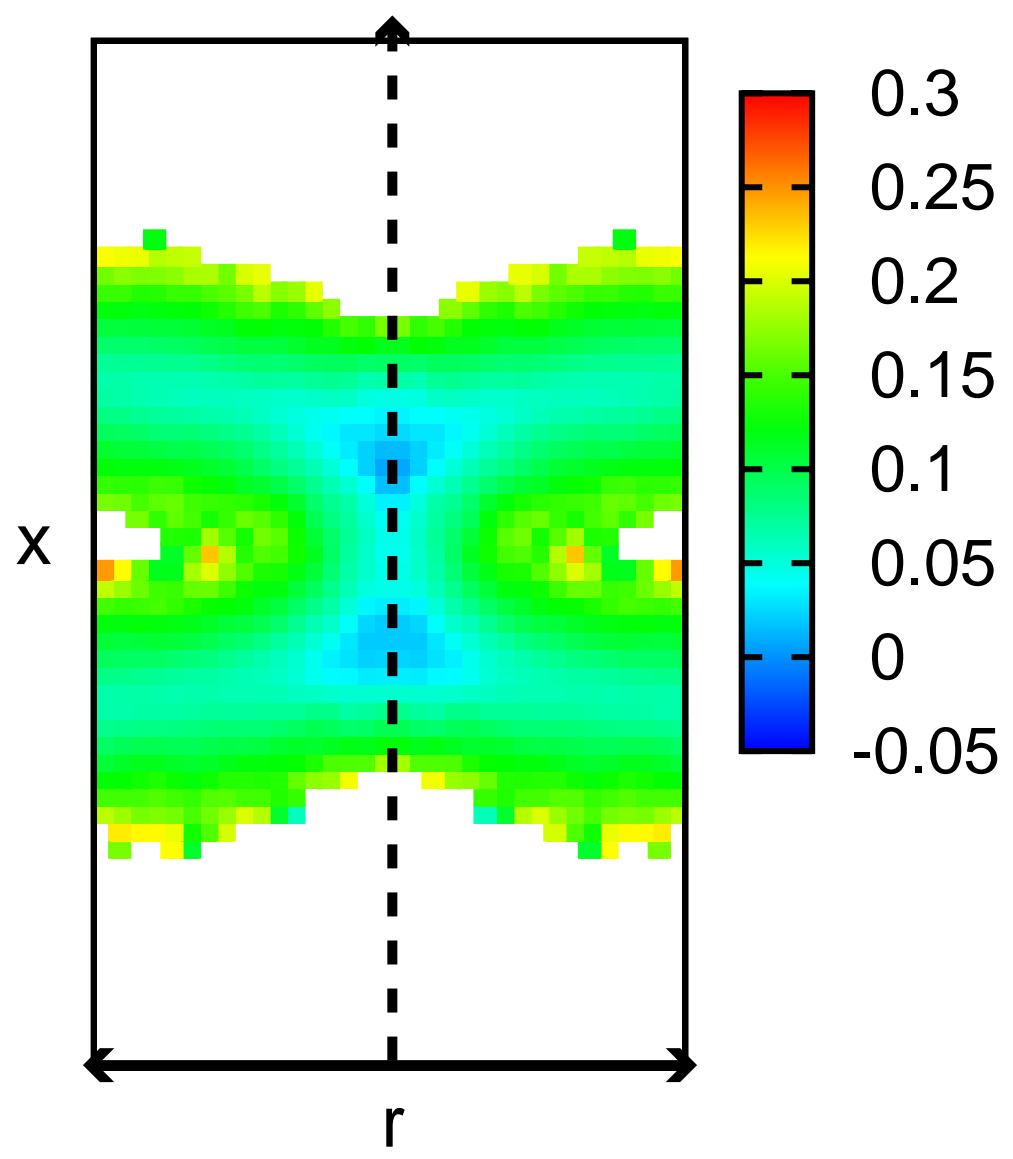

Figure 5.12: The normalized difference of molecular density distribution between both the molecular species $N_{A}=29$ and $N_{A}=28$, defined in eq. (5.8), measured at $\left(\mu_{\alpha}-\mu_{\gamma}\right) / k_{B} T=$ -2 . These data are radially averaged around the central axis of the stalk (see also Fig. 5.5). Molecules of $\alpha$-species, i.e. more asymmetric molecular architecture $N_{A}=29$, concentrate around the stalk. 


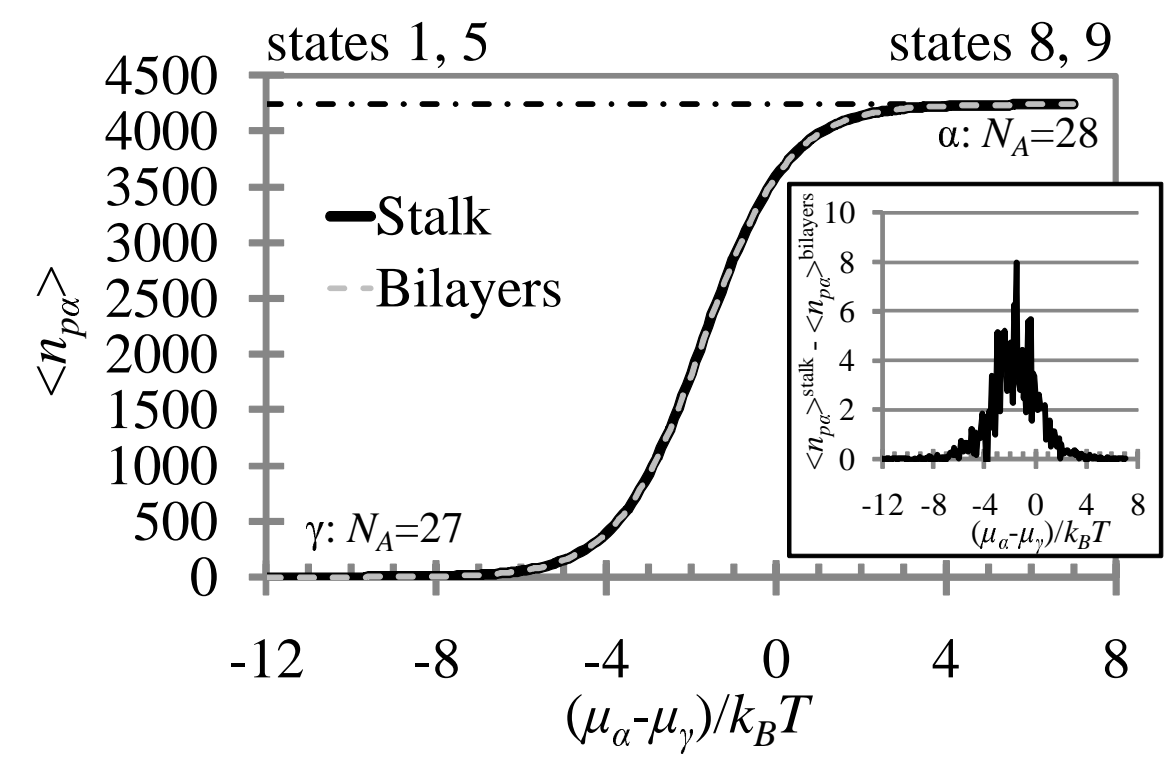

Figure 5.13: Semigrand canonical ensemble simulation results between $N_{A}=28$ and $N_{A}=27$. The main plate shows $\left\langle n_{p \alpha}\right\rangle_{T, \mu_{\alpha}, \mu_{\gamma}, V, n_{p}}$ as a function of the chemical potential difference, $\left(\mu_{\alpha}-\mu_{\gamma}\right) / k_{B} T$, for the stalk morphology and the two apposed bilayers, which are plotted by a black solid line and a broken grey line respectively. Both the branches are set in the interval $-12.0 \leq\left(\mu_{\alpha}-\mu_{\gamma}\right) / k_{B} T \leq 7.0$ and discretised homogeneously into 191 sampling points. $\alpha$-species is the molecules with $N_{A}=28$ and $\gamma$-species $N_{A}=27$. The smooth form of these lines indicates the absence of the first-order transition. The black chain line shows the total number of the molecules, $n_{p}=4240$. The inset presents the difference between the data in the main panel, $\Delta\left\langle n_{p \alpha}\right\rangle_{T, \mu_{\alpha}, \mu_{\gamma}, V, n_{p}}$. 


\subsection{Grand potential of the stalk}

When the stalk bridges a pair of large bilayers, unperturbed regions of the bilayers, i.e. regions far away from the stalk, work as the reservoir of amphiphiles. This situation means that the tension of the bilayers is a constant before and after the stalk formation. In such systems, the excess grand potential of the stalk at the constant chemical potential $\mu$, denoted by $\Delta \Phi_{G}(\mu)$, describes the stalk stability. In other words, $\Delta \Phi_{G}(\mu)$ corresponds to the stalk stability at the constant tension of the bilayers. As a result, the number of molecules in the stalk morphology is larger than the number in the two apposed bilayers.

In this section, we calculate this excess grand potential of the stalk in Grand canonical ensemble simulations discussed in section 3.3. We still use the system box given in Table 5.2. However, the number of molecules is not a constant in this simulation. The grand potential at chmical potential $=\mu$ is denoted by $\Phi_{G}^{\text {bilayers }}(\mu)$ and $\Phi_{G}^{\text {stalk }}(\mu)$ for the two apposed bilayers and the stalk morphology respectively.

Grand potential is calculated via the thermodynamic integration in the grand canonical ensemble, eq. (4.20). At $N_{A}=28$, the integration of $\left\langle n_{p}\right\rangle_{\mu, T, V}$, obtained in these simulations, from the state 1 to some chemical potential $\mu$ yields the grand potential difference between these two states, $\Phi_{G}^{\text {bilayers }}(\mu)-\Phi_{G}^{\text {bilayers }}\left(\mu_{1}\right)$. In the same way, the grand potential difference for the stalk morphology is also calculated, i.e. $\Phi_{G}^{\text {stalk }}(\mu)-\Phi_{G}^{\text {stalk }}\left(\mu_{5}\right)$. Comparing these results and adding $\Delta F$, we obtain the excess grand potential of the stalk at the fixed $\mu, \Delta \Phi_{G}(\mu)=\Phi_{G}^{\text {stalk }}(\mu)-\Phi_{G}^{\text {bilayers }}(\mu)$, at $N_{A}=28$. For the single component systems with $N_{A}=29$, instead of the states 1 and 5, the states 6 and 7 are chosen as the reference respectively.

In our grand canonical ensemble simulation, $10 \%$ of the total simulation steps is chosen as the trial particle exchange between the system and the reservoir on average. the number of trial positions in constructing one segment, denoted by $k$ in our algorithm (see section 3.3.1), is fixed at 5 .

The tension of the bilayer membranes, $\sigma(\mu, T, V)$, is calculated via the grand canonical ensemble simulations of the single isolated bilayer according to the Gibbs adsorption isotherm,

$$
\sigma(\mu, T, V)=\frac{\Phi_{G}^{\text {single }}(\mu)-\Phi_{G}^{\text {single }}\left(\mu_{0}\right)}{L_{y} L_{z}}=-\int_{\mu_{0}}^{\mu} d \mu^{\prime} \frac{\left\langle n_{p}\right\rangle_{\mu^{\prime}, T, V}^{\text {single bilayer }}}{L_{y} L_{Z}}
$$

where $\Phi_{G}^{\text {single }}(\mu)$ denotes the grand potential of the single bilayer and $\left\langle n_{p}\right\rangle_{\mu^{\prime}, T, V}^{\text {single bilayer }}$ the average $n_{p}$ of the single bilayer.

The calculated chemical potentials of the single tensionless bilayer, the stalk morphology, and the two apposed bilayers in the canonical ensemble, which are required in these grand canonical ensemble simulations as the reference of TDI, via Rosenbluth sampling are shown in Table 5.4 and 5.5. These chemical potentials are referred to the chemical potential of the single tensionless bilayer, $\mu_{0}$, by eq. (3.62). In the canonical ensemble, the chemical potential of the stalk morphology is less than the one of the two apposed bilayers, i.e. $\mu_{5}<\mu_{1}$ and $\mu_{7}<\mu_{6}$, because 
the regions on the bilayers far away from the stalk are thinner than those in the two-bilayers morphology.

Table 5.4: Chemical potentials measured via Rosenbluth sampling on each state at $N_{A}=$ 28. The chemical potentials are referred to the chemical potential of the single tensionless bilayer, $\mu_{0}$, by eq. (3.62).

\begin{tabular}{cccc}
\hline State index $i$ & $n_{p} R_{e}^{3} / V$ & $\beta \mu_{i}^{\mathrm{ex}}$ & $\beta \mu_{i}-\beta \mu_{0}$ \\
\hline 0 & 11.66 & -37.73983772 & 0 \\
1 & 11.77777778 & -37.71120594 & 0.038682113 \\
5 & 11.77777778 & -37.74760927 & 0.002278791 \\
\hline
\end{tabular}

Table 5.5: Chemical potentials measured via Rosenbluth sampling on each state at $N_{A}=$ 29. The chemical potentials are referred to the chemical potential of the single tensionless bilayer, $\mu_{0}$, by eq. (3.62).

\begin{tabular}{cccc}
\hline State index $i$ & $n_{p} R_{e}^{3} / V$ & $\beta \mu_{i}^{\mathrm{ex}}$ & $\beta \mu_{i}-\beta \mu_{0}$ \\
\hline 0 & 12.06 & -39.57944964 & 0 \\
6 & 11.77777778 & -39.64344754 & -0.087677575 \\
7 & 11.777777778 & -39.72675926 & -0.170989294 \\
\hline
\end{tabular}

$\left\langle n_{p}\right\rangle_{\mu, T, V}$ of the single component system composed of molecules with $N_{A}=$ 28 in the grand canonical ensemble are presented in Fig. 5.14. From these data, tension of the single bilayer and $\Delta \Phi_{G}(\mu)$ are determined. We observe that the excess number of amphiphiles in the stalk, $\Delta\left\langle n_{p}\right\rangle=\left\langle n_{p}\right\rangle^{\text {stalk }}-\left\langle n_{p}\right\rangle^{\text {bilayers }}$ is on the order of a few tens and it decreases as we increase the chemical potential, $\mu$, or decrease the tension of the membrane, $\sigma$. We also note that the area density of amphiphiles in the single bilayer is slightly larger than in the two, apposed bilayers. We speculate that this effect mirrors the repulsive interactions between the apposed bilayers.

The grand potential of the stalk $\Delta \Phi_{G}$ and the chemical potential is plotted as the functions of $\sigma$ in Fig. 5.15. We observe that $\Delta \Phi_{G}$ increases with membrane tension. At $\mu=\mu_{0}$, we obtain $\Delta \Phi_{G}=16.2 k_{B} T$, which is consistent with the observation that the stalk disappears in long simulation runs i.e. metastability of the stalk.

In Fig. 5.16, the difference between the molecular density distribution of hydrophobic $A$-species in the stalk morphology at $\mu-\mu_{0}=0.100 k_{B} T$ and the one at $\mu-\mu_{0}=-0.100 k_{B} T$ is shown. This graph indicates that, in the vicinity of the stalk, the material mostly increases in the stalk itself with chemical potential. This results in the stability of the stalk at high $\mu$, i.e. a thick stalk. On the other hand outside the vicinity of the stalk, i.e. in the bilayers, the number of lipids homogeneously increases. Due to this result, $\Delta\left\langle n_{p}\right\rangle$ decreases at high chemical potential.

The simulation results at $N_{A}=29$ in the grand canonical ensemble, i.e. the number of lipids in the system $\left\langle n_{p}\right\rangle$, the excess free energy of a stalk $\Delta \Phi_{G}$, and the tension of the single isolated bilayer $\sigma$ are shown in Figs. 5.17 and 5.18. These 


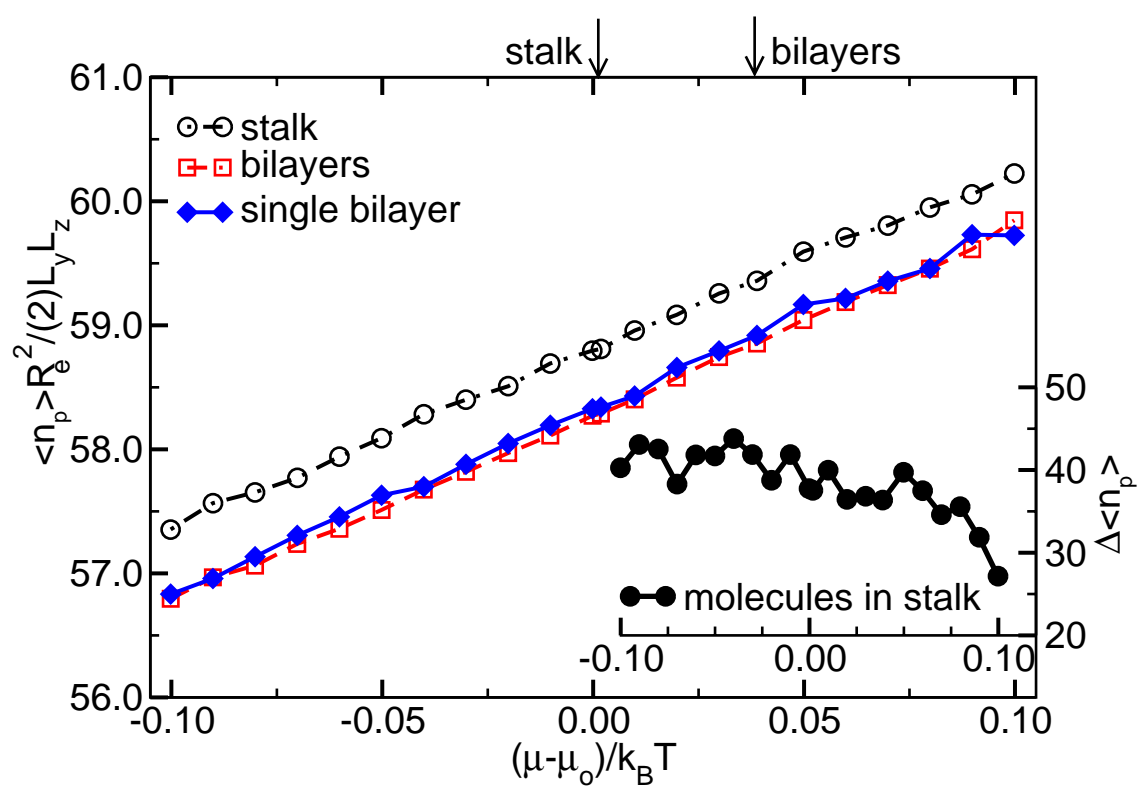

Figure 5.14: Simulation resutls at $N_{A}=28$ in the grand canonical ensemble. Number of molecules per area in a single bilayer (diamonds), two-apposed bilayers (squares), and the stalk-morphology (circles) as a function of the chemical potential referred to the chemical potential, $\mu_{0}$, of the tensionless state. The inset depicts the excess number of molecules of the stalk, $\Delta\left\langle n_{p}\right\rangle=\left\langle n_{p}\right\rangle^{\text {stalk }}-\left\langle n_{p}\right\rangle^{\text {bilayers }}$ as a function of $\mu-\mu_{0}$.

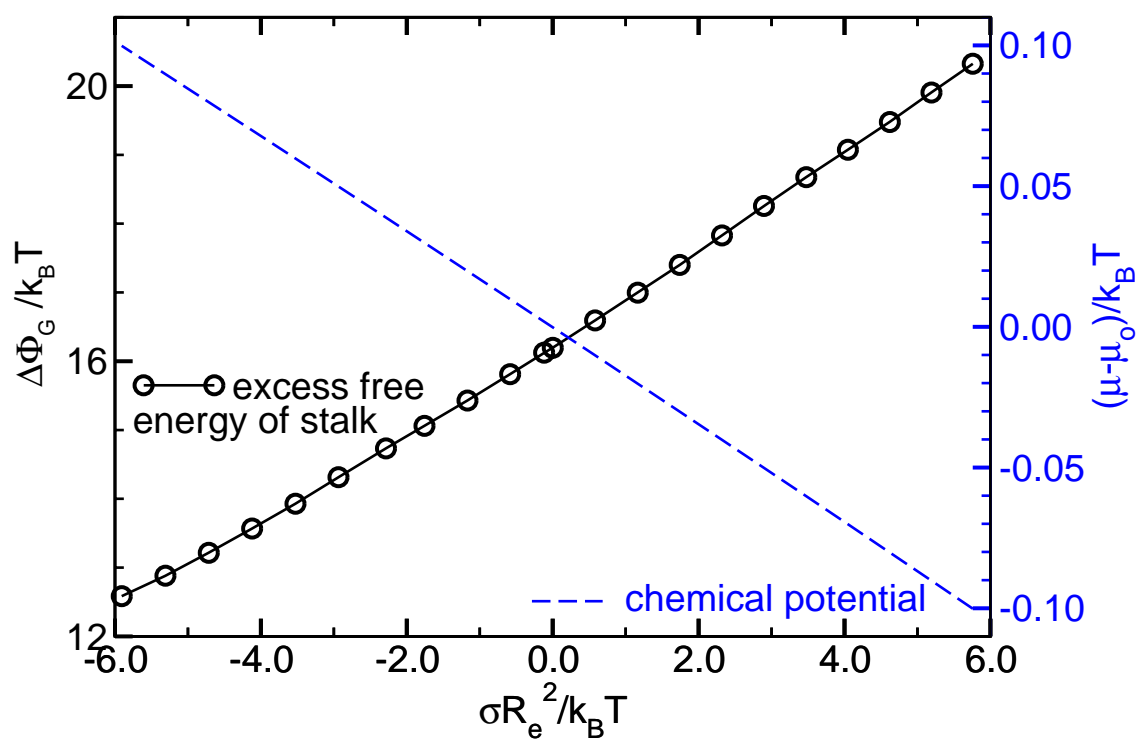

Figure 5.15: Excess grand canonical potential, $\Delta \Phi_{G}$ (circles), of the stalk as a function of the membrane tension, $\sigma$, at $N_{A}=28$. On the right hand side, we show the dependence of the chemical potential, $\mu$ (dashed line), on the membrane tension, $\sigma$. The unit of the tension, $k_{B} T / R_{e}^{2}=7.83 \times 10^{-4}\left(\mathrm{~J} / \mathrm{m}^{2}\right)$ at $T=300(\mathrm{~K})$. 


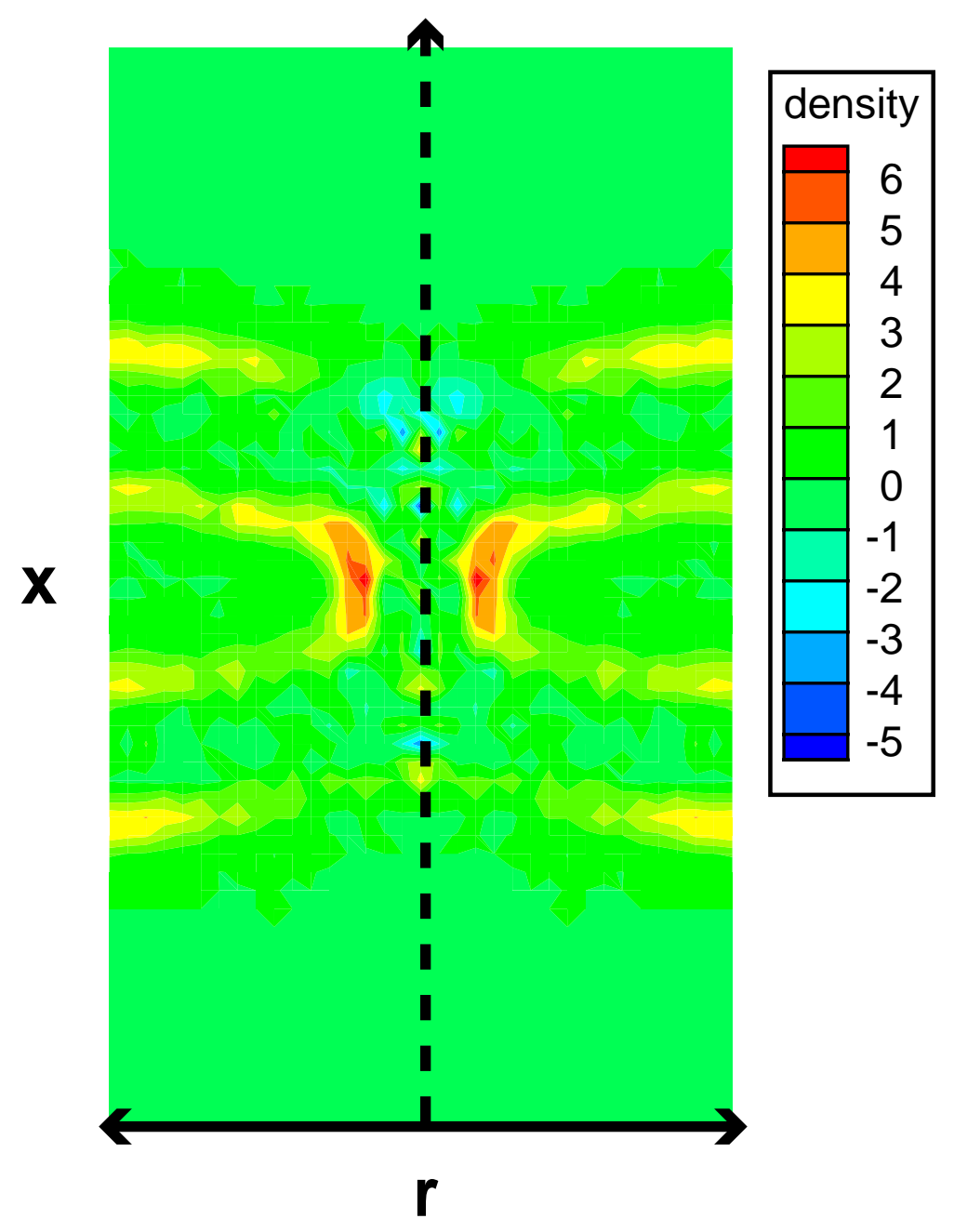

Figure 5.16: $2 \mathrm{D}$ contour plot of the difference between the molecular density distribution of hydrophobic $A$-species in the stalk morphology at $N_{A}=28$, radially averaged around the central axis of the stalk, in the grand canonical ensemble at $\mu-\mu_{0}=0.100 k_{B} T$ and the one at $\mu-\mu_{0}=-0.100 k_{B} T$ (see also Fig. 5.5). The graph shows the molecular density distribution of tails at $\mu-\mu_{0}=0.100 k_{B} T$ minus the distribution at $\mu-\mu_{0}=-0.100 k_{B} T . r$ and $x$ denote the radial distance from the central axis and the coordinate along the membrane normal respectively. This result indicates that, in the vicinity of the stalk, the materials mostly increase on the stalk itself with chemical potential. On the other hand, outside this vicinity, i.e. in bilayers, the number of molecules homogeneously increases.

data indicate the similar dependence on the chemical potential to the results at $N_{A}=28$. We also note that the excess number of amphiphiles in the stalk is larger than the result at $N_{A}=28$ and that the spontaneous stalk formation is observed in the system at $N_{A}=29$. 


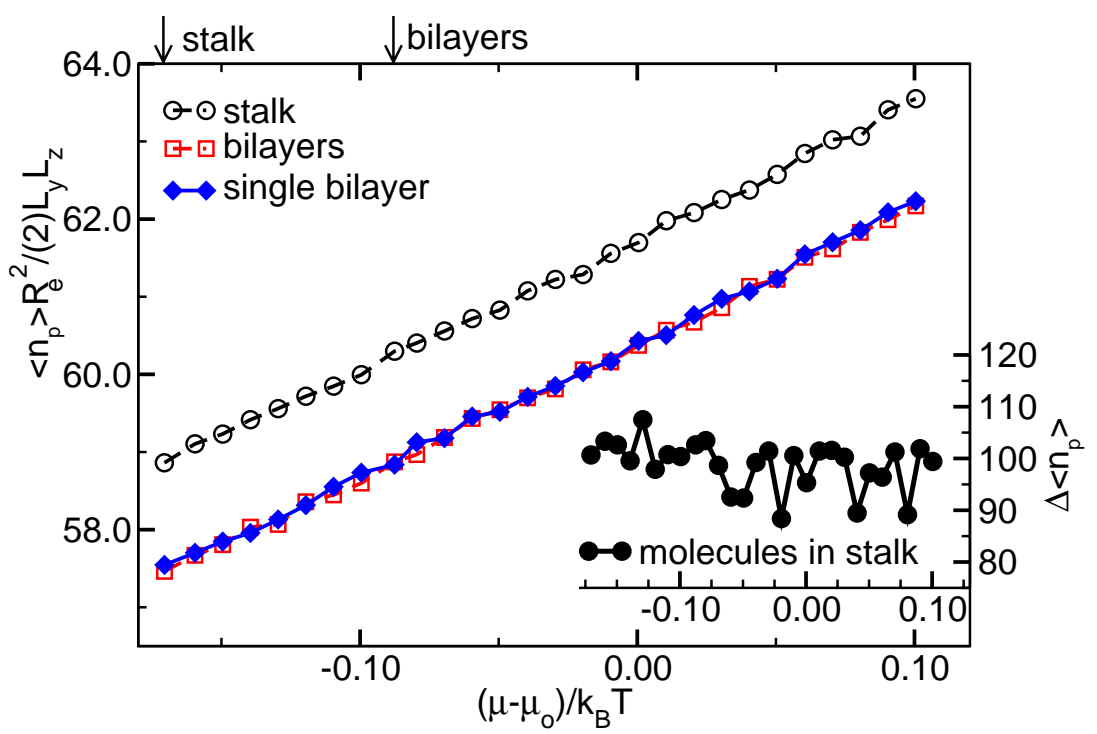

Figure 5.17: Simulation resutls at $N_{A}=29$ in the grand canonical ensemble. Number of molecules per area in a single bilayer (diamonds), two-apposed bilayers (squares), and the stalk-morphology (circles) as a function of the chemical potential referred to the chemical potential, $\mu_{0}$, of the tensionless state. The inset depicts the excess number of molecules of the stalk, $\Delta\left\langle n_{p}\right\rangle=\left\langle n_{p}\right\rangle^{\text {stalk }}-\left\langle n_{p}\right\rangle^{\text {bilayers }}$ as a function of $\mu-\mu_{0}$.

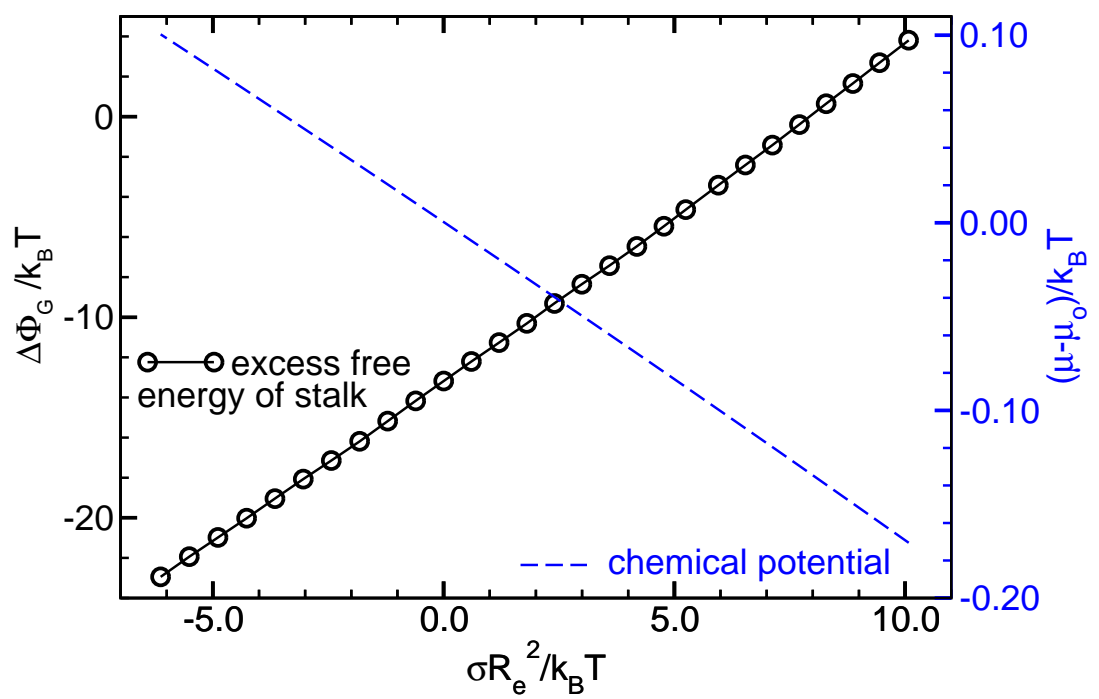

Figure 5.18: Excess grand canonical potential, $\Delta \Phi_{G}$ (circles), of the stalk as a function of the membrane tension, $\sigma$, at $N_{A}=29$. On the right hand side, we show the dependence of the chemical potential, $\mu$ (dashed line), on the membrane tension, $\sigma$. 


\section{Chapter 6}

\section{Conclusions}

We have devised a general computational strategy for computing free energies of self-assembling systems $[1,36]$. It has been employed to determine the excess free energy of a stalk that bridges a pair of apposed bilayer membranes, using a thermodynamic integration scheme based on external fields. The technique relies on reversibly transforming one self-assembled structure into another by substituting the non-bonded interactions by external, ordering fields. To ensure reversibility, these external, ordering fields have to be chosen as to generate the structure of the self-assembled system in a system, where the non-bonded interactions have been turned off, i.e., an ideal gas. Applications to dense polymer system studying fluctuation-induced, first-order transition between a disordered and a lamellar phases in diblock copolymers [36] and the free energies of grain boundariew [37] demonstrated that the external field can be estimated from mean field theory. For the case of the strongly fluctuating bilayer systems considered in the main part of this work the mean field approximation fails and we have devised a numerical strategy for calculating the external fields.

Along the thermodynamic integration paths, the excess Helmholtz free energy of the stalk has accurately been calculated with the use of expanded ensembles simulation. This method quantitatively verifies the absence of the first order transition along the paths.

The simulations have been performed using a solvent-free coarse-grained model which reduces the computational time and facilitates the Monte Carlo simulations due to the reduced number of the degrees of freedom and the soft interaction. This allows, for example, for a very accurate measurment of chemical potential required for the simulations of lipid bilayers in the grand canonical ensemble. The Widom's insertion and deletion schemes that we used would not be efficient in a system with hard interactions. However the proposed TDI approach can be used in context of a broad scope of different models (e.g. Lennard-Jones potentials) and simulation techniques such as DPD, conventional molecular dynamics, and single-chain-inmean-field-simulations [17]. For example we have utilized this technique within the framework of a Flory-Huggins type density functional to compute the free en- 
ergy of T-junctions and the free energy cost of the surface reconstruction of the soft morphologies in thin films of lamella forming diblock copolymers assembled on patterned substrates.

Focusing on the simulations of bilayers, our modeling approach provided information on the stalk structure which consists of at most one hundred amphiphilic molecules, a few $\%$ of the total molecules in our system. This in combination with the fact that the free energy differences between different morphololgies in soft-matter systems typically are small, the accurate measurement of the free energy is a computational challenge. In this work, we have utilized a combination of sophisticated simulation techniques (e.g. expanded ensemble and reweighting methods $[1,65,66])$ in conjunction with thermodynamic integration which are well suitable for parallel computing [37]. We obtained an accuracy of $4 k_{B} T$ in the free energy calculation, which corresponds to the relative accuracy of $10^{-5}$, within our computational resources.

Once the excess Helmholtz free energy of the stalk, $\Delta F$, is determined in the canonical ensemble, the dependence of the excess free energies in the canonical and grand canonical ensemble, $\Delta F$ and $\Delta \Phi_{G}$, on the molecular architecture and the chemical potential $\mu$ can be obtained with a relatively low computational cost. The dependence of the excess free energy $\Delta \Phi_{G}$ on the chemical potential can be utilized to extract the dependence of $\Delta \Phi_{G}$ on the membrane tension, $\sigma$; an information which can provide a link to experiments.

We have determined the excess free energy of a stalk connecting two tensionless bilayer membranes to be $\Delta \Phi_{G}=16.2 k_{B} T$ with $N_{A}=28$. This free energy is lower than earlier estimates based on the phenomenological theory, whereas it is consistent with the results in self-consistent field calculations [30]. This result is particularly notable owing to significant differences in the microscopic structures between our solvent-free model and the model of the self-consistent field calculations, e.g., we use an implicit solvent while the self-consistent field model represents the solvent by homopolymers. This finding suggests that the excess free energy of the stalk is not very sensitive to the specific interactions of the model and that the results for our simple coarse-grained model are also relevant for synthetic or biological bilayer membranes.

When the membrane tension increases, we observe that the number of molecules, of which the stalk is composed, increases and, in turn, the excess free energy, $\Delta \Phi_{G}$ also increases slightly. This finding differs from the results of self-consistent field calculations [30], which observe that the free energy of the stalk is almost independent from the membrane tension or decreases with $\sigma$. In the vicinity of the stalk, the distribution of the molecular density changes mostly in the stalk itself with the tension $\sigma$. This accounts for the high stability of the stalk at low $\sigma$. We observe that the stalk becomes unstable for more symmetric molecules in agreement with self-consistent field calculations [30]. We also observe that in lipid mixtures, comprised of two species with different molecular asymmetries, the more asymmetric species segregates to the stalk.

In this work we have focused on the study of the free energy of the stalk inter- 
mediate without considering its further evolution into a fusion pore. In this scope, it would be interesting to investigate potential pathways which the fusion process can follow after the stalk structure is formed. This can be performed via Monte Carlo simulations utilizing moves that mimic a realistic chain dynamics. In case some specific fusion intermediate structures are indentified rluving the simulations, a TDI technique similar to the one developed in this work could be used to estimate the free energy of these fusion intermediates and exploring the observed pathway. An additional topic that we would like to address in the future refers to investigating further the influence of the chain architecture on the stalk stability and the kinetics of its formation. For instance, it has been argued [67] that the double tail structure, a common feature of many lipid molecules, can play an important roll during stalk nucleation. 


\section{Appendix A}

\section{Gaussian chain}

We have simulated molecules in a coarse-grained molecular scale using beadspring polymer model. This model is based on the structure of ideal polymers in a coarse-grained scale. In this appendix, we begin our discussion with the microscopic polymer structure and introduce the bead-spring model, also called Gaussian chain. It is shown that any linear polymers result in Gaussian chain at high temperature.

\section{A.1 Microscopic polymer structure}

We discuss the micro structural character of a linear polymer with a carbon backbone $[45,68,69]$, e.g. polyethylene sketched in Fig. A.1 as a particular example. The number of repeating units in a polymer, $N_{0}$, is called the degree of polymerization or the polymerization degree. The material for a polymer, e.g. ethylene for polyethylene, is called a monomer.<smiles>C=C</smiles>

(b)

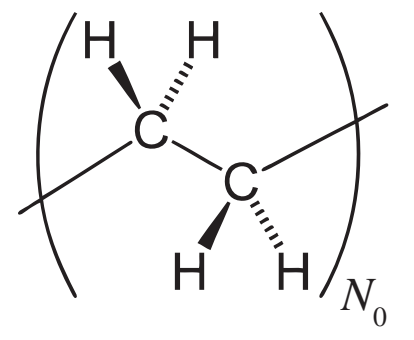

Figure A.1: Chemical structures of ethylene, (a), and polyethylene, (b), as a particular example of a linear polymer. Ethylene is polymerized into polyethylene and composes this repeating unit in polyethylene. In this case, ethylene is called a monomer. The number of repeating units, $N_{0}$, is called the degree of polymerization or the polymerization degree. Steric structure of the carbon backbone in a linear polymer is presented in Fig. A.2.

Figure A.2 presents the steric structure of the carbon backbone in a linear polymer. The angle $\theta$ between a pair of neighboring C-C bonds in a polymer, the 
valence angle, usually ranges approximately from $50^{\circ}$ to $80^{\circ}$ in response to the chemical properties of the polymer and remains fixed through the entire polymer chain. Due to the axial symmetry, rotation around this bond only slightly changes the energy.

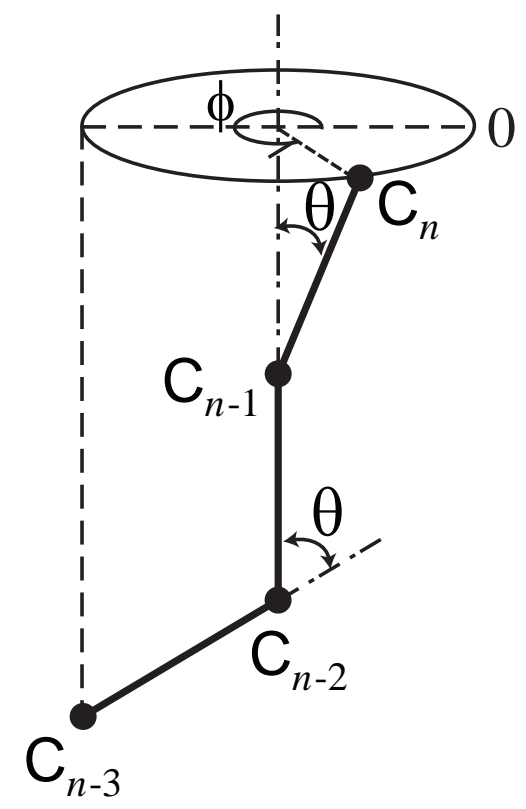

Figure A.2: Steric structure of the carbon backbone in a linear polymer on an atomic scale. Thick solid lines represent covalent bonds between adjacent carbon atoms. $\phi=0^{\circ}, 120^{\circ}$, and $240^{\circ}$ correspond to trans, gauche ${ }^{+}$, and gauche $e^{-}$respectively (see Fig. A.3).

When the polymer is formed by adding one carbon $(n)$ to 3 fixed carbon atoms $(n-3, n-2, n-1)$, the internal rotation angle along the conical surface, $\phi$, is defined (see Fig. A.2). The vertex angle of the cone always equals $2 \theta$. The energy between the carbon atoms is determined by this internal rotation angle $\phi$. This energy function of $\phi$ (shown in Fig. A.3) shows three local minimum points at $\phi=0^{\circ}, 120^{\circ}$, and $240^{\circ}$, which correspond to the three steric structures i.e. trans, gauche $e^{+}$, and gauche $^{-}$respectively (see Fig. A.2). At $\phi=0^{\circ}$, in trans, the energy between the carbon atoms takes the global minimum value. Thus, in the ground state all the bonds are fixed at $\phi=0^{\circ}$ and the whole polymer is arranged in a planar transzigzag conformation, shown in Fig. A.4, whereas the thermal energy induces the local minimum state in the energy between the carbon atoms, that is, gauche. In view of a larger scale depicted in Fig. A.5, at low temperature rigid straight conformation appears though at high temperature the whole polymer adopts a flexible structure.

We can construct a simplified model that captures these microscopic characteristics of the polymer chain e.g. Rotational Isomeric State (RIS)-model. In RISmodel, all the C-C bonds are fixed to the same length and the torsion angles are allowed to take only trans and gauche angles. In other words, the internal rotation 
Energy between the carbon atoms as a function of internal rotation angle $\phi$

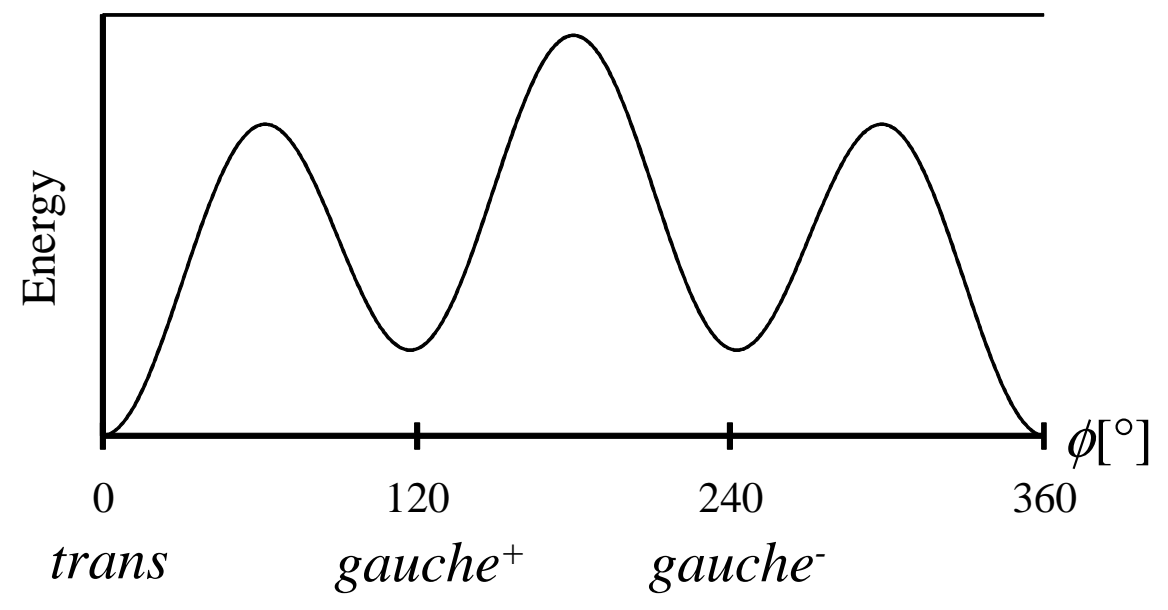

Figure A.3: Energy between the carbon atoms as a function of internal rotation angle $\phi$. The steric structure of the polymer at $\phi=0^{\circ}, 120^{\circ}$, and $240^{\circ}$ correspond to trans, gauche ${ }^{+}$, and gauche $e^{-}$respectively.

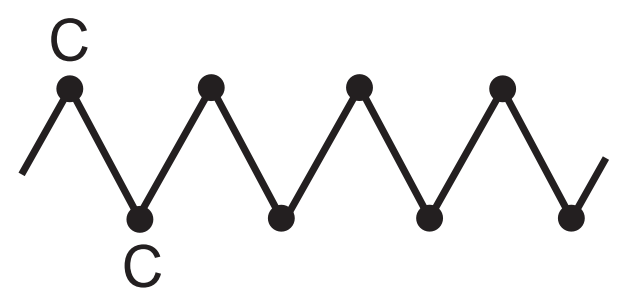

Figure A.4: Planar trans-zigzag conformation, the ground state of the polymer.

(a)

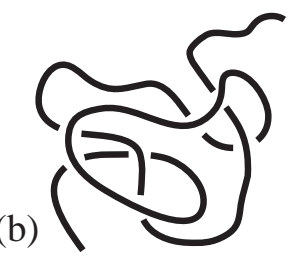

Figure A.5: Polymer conformation on a large scale, (a), in the ground state and, (b), at high temperature. Structure of the polymer in figure (a) is consistent with planar trans-zigzag conformation in a small scale (see Fig. A.4).

angle is limited to $\phi=0^{\circ}, 120^{\circ}$, and $240^{\circ}$, the three local minimum points. This model can be applied to simulation of the coarse-grained polymer systems, which retain the microscopic structural property. 


\section{A.2 Gaussian polymer chain}

Next, the statistics of a single ideal linear polymer chain is discussed in this section [70]. An ideal polymer is defined as a molecule interacting only via intramolecular interaction e.g. bonding, bending, and torsion. Other interactions between monomers, e.g. non-bonded Lennard-Jones interaction, Coulomb interaction, and excluded volume interaction, are not counted in this polymer (see also section 3.2). Ideal polymers are suited to study the effect of intra-molecular connection between monomers on the physical properties in polymeric systems.

\section{A.2.1 Persistence length}

We assume that our system consists of an ideal polymer at the high temperature at which the system can move away from the ground state. Along the polymer chain, a pair of small segments are taken. When the contour length along the polymer between this pair is small, the orientations of the polymer chain on these segments are correlated. However, at the large contour length this correlation, i.e. the memory of chain orientation, vanishes and the segments are oriented independently. When this memory is lost at some contour distance on average, this length, denoted by $l_{p}$, is called persistence length of the polymer. In a scale smaller than $l_{p}$ the polymer takes stiff conformation, e.g. shown in Fig. A.4, whereas in a scale larger than $l_{p}$ flexible conformation, e.g. in Fig. A.5 (b).

\section{A.2.2 End-to-end vector}

We are coarse-graining this ideal polymer in a length scale larger than $l_{p}$. In our coarse-grained model, the linear polymer is reduced to $N$ linearly linked segments, which is sketched in Fig A.6 (a) and (b). Here bond vectors, i.e. the relative coordinates between adjacent coarse-grained segments, are defined as (see also section 3.2):

$$
\boldsymbol{u}_{j}:=\boldsymbol{r}_{j+1}-\boldsymbol{r}_{j}, \quad 1 \leq j \leq N_{\text {bond }},
$$

where $\boldsymbol{r}_{j}$ is the position of the $j$-th segment and $N_{\text {bond }}:=N-1$ denotes the number of the bonds in the polymer. Obviously, probability density of each bond vector, denoted by $P_{1}\left(\boldsymbol{u}_{j}\right)$, is statistically independent and isotropically distributed,

$$
P_{1}\left(\boldsymbol{u}_{j}\right)=P_{1}\left(\left|\boldsymbol{u}_{j}\right|\right) .
$$

Probability density of all the bonds in the polymer, $\left(\boldsymbol{u}_{1}, \ldots, \boldsymbol{u}_{N-1}\right)$, is:

$$
P_{N_{\text {bond }}}\left(\boldsymbol{u}_{1}, \ldots, \boldsymbol{u}_{N-1}\right)=\prod_{j=1}^{N-1} P_{1}\left(\left|\boldsymbol{u}_{j}\right|\right) .
$$


(a)

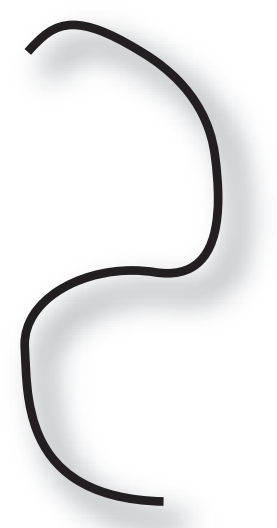

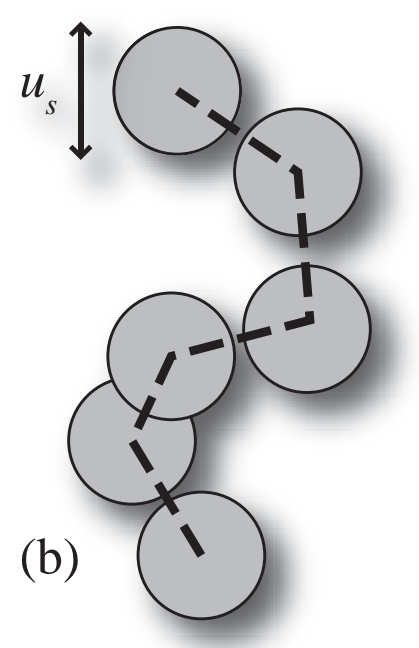

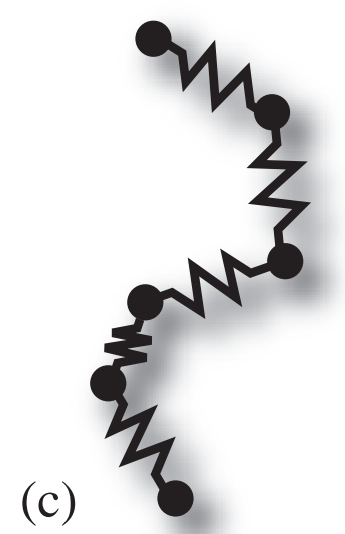

Figure A.6: A linear ideal polymer, (a), is coarse-grained into $N$ linearly linked segments in larger scale than $l_{p}$, (b). The coarse-grained segments are drawn by grey spheres. The broken line represents the links, i.e. molecular configuration, between these coarse-grained segments. Gaussian chain, (c), is obtained by replacing these bonds with the harmonic springs, eq. (A.5). The length $u_{s}$ corresponds to both the coarse-grained segment size and the average spring length.

Hereafter, we discuss one structural property of the polymer, i.e. the end-to-end vector,

$$
\boldsymbol{R}:=\boldsymbol{r}_{N}-\boldsymbol{r}_{1}=\sum_{j=1}^{N-1} \boldsymbol{u}_{j}
$$

We can determine the probability density of $\boldsymbol{R}$, denoted by $P(\boldsymbol{R})$, using Dirac delta, $\delta$.

$$
\begin{aligned}
P(\boldsymbol{R}) & =\int d \boldsymbol{u}_{1} \cdots d \boldsymbol{u}_{N-1} P_{N_{\text {bond }}}\left(\boldsymbol{u}_{1}, \ldots, \boldsymbol{u}_{N-1}\right) \delta\left(\sum_{j=1}^{N-1} \boldsymbol{u}_{j}-\boldsymbol{R}\right) \\
& =\int d \boldsymbol{u}_{1} \cdots d \boldsymbol{u}_{N-1}\left(\prod_{j=1}^{N-1} P_{1}\left(\left|\boldsymbol{u}_{j}\right|\right)\right) \frac{1}{(2 \pi)^{3}} \int d \boldsymbol{q} \exp \left[i \boldsymbol{q} \cdot\left(\sum_{j=1}^{N-1} \boldsymbol{u}_{j}-\boldsymbol{R}\right)\right] \\
& =\frac{1}{(2 \pi)^{3}} \int d \boldsymbol{q} \exp [-i \boldsymbol{q} \cdot \boldsymbol{R}]\left(\hat{P}_{1}(\boldsymbol{q})\right)^{N-1} .
\end{aligned}
$$

where $\boldsymbol{q}$ denotes the wave number vector and $\hat{P}_{1}(\boldsymbol{q})$ does the Fourier transformation of $P_{1}\left(\left|\boldsymbol{u}_{j}\right|\right)$,

$$
\hat{P}_{1}(\boldsymbol{q}):=\int d \boldsymbol{u} P_{1}(u) \exp [i \boldsymbol{q} \cdot \boldsymbol{u}], \quad \boldsymbol{u}:=\boldsymbol{u}_{j} \text { and } u:=|\boldsymbol{u}| .
$$

Equation (A.2) represents the inverse Fourier transformation of $P(\boldsymbol{R})$. 
By performing this inverse Fourier transformation,

$$
P(\boldsymbol{R})=\left(\frac{3}{2 \pi N_{\text {bond }}\left\langle u^{2}\right\rangle}\right)^{3 / 2} \exp \left[-\frac{3|\boldsymbol{R}|^{2}}{2 N_{\text {bond }}\left\langle u^{2}\right\rangle}\right],
$$

where $\left\langle u^{2}\right\rangle$ denotes the mean square of $u$. The length $u_{s}:=\sqrt{\left\langle u^{2}\right\rangle}$ corresponds to the segment size. This relation eq. (A.3) indicates that, in a length scale larger than $l_{p}$, the end-to-end vector of ideal polymers is distributed according to Gaussian distribution regardless of model details and chemical structure of polymers. This property results from the central limit theorem. The probability density of each bond vector is also calculated by the inverse Fourier transformation,

$$
P_{N_{\text {bond }}}\left(\boldsymbol{u}_{1}, \ldots, \boldsymbol{u}_{N-1}\right)=\left(\frac{3}{2 \pi\left\langle u^{2}\right\rangle}\right)^{\frac{3}{2} N_{\text {bond }}} \exp \left[-\frac{3}{2\left\langle u^{2}\right\rangle} \sum_{j=1}^{N_{\text {bond }}}\left|\boldsymbol{u}_{j}\right|^{2}\right] .
$$

The detail calculation for equations (A.3) and (A.4) is shown in section A.2.5.

\section{A.2.3 Gaussian chain}

When segments are bonded by harmonic springs with the spring constant $k_{\text {spring, }}$,

$$
k_{\text {spring }}=\frac{3 k_{B} T}{\left\langle u^{2}\right\rangle}, \quad k_{B} T:=\text { thermal energy, }
$$

the probability density of each bond vector in canonical ensemble is equivalent to eq. (A.4) (see section 3.2.2-b). This indicates that ideal polymers in the coarsegrained scale larger than $l_{p}$ can be modelled by a sequence of these harmonic springs that connect the segments.

This polymer model is called Gaussian chain or bead-spring model, which is schematically presented in Fig A.6 (c). The length $u_{s}$ corresponds to both the coarse-grained segment size and the average length of one spring in Gaussian chain.

\section{A.2.4 Size of Gaussian chain}

From the probability density eq. (A.3), mean square end-to-end distance of the Gaussian chain is determined,

$$
\left\langle R^{2}\right\rangle=\int d \boldsymbol{R} P(\boldsymbol{R})|\boldsymbol{R}|^{2}=N_{\text {bond }}\left\langle u^{2}\right\rangle, \quad R_{e}:=\sqrt{\left\langle R^{2}\right\rangle} .
$$

The length $R_{e}$ corresponds to the size of Gaussian chain.

For example in the cubic lattice model with lattice constant $b,\left\langle R^{2}\right\rangle=N_{\text {bond }} b^{2}$. This is consistent with results for random walkers on the lattice. 


\section{A.2.5 Derivation of Gaussian chain}

In order to derive equations (A.3) and (A.4), we first calculate $\hat{P}_{1}(\boldsymbol{q})$ and perform the inverse Fourier transformation eq. (A.2).

In our polymeric system, the polymerization degree $N$ is far larger than 1 . At such large $N$, physical properties of the polymer chain at long distance are important. This region corresponds to small $|\boldsymbol{q}|$. When we study the statistics at this long distance, using Taylor expansion of $\exp [i \boldsymbol{q} \cdot \boldsymbol{u}]$ around $\boldsymbol{q}=0$ and the spherical coordinate system of $\boldsymbol{u}$, we obtain

$$
\begin{aligned}
\hat{P}_{1}(\boldsymbol{q}) & =\int d \boldsymbol{u} P_{1}(u) \exp [i \boldsymbol{q} \cdot \boldsymbol{u}] \\
& \cong \int d \boldsymbol{u} P_{1}(u)\left(1+i \boldsymbol{q} \cdot \boldsymbol{u}-\frac{1}{2}(\boldsymbol{q} \cdot \boldsymbol{u})^{2}\right)=1-\frac{1}{2} \int d \boldsymbol{u} P_{1}(u)(\boldsymbol{q} \cdot \boldsymbol{u})^{2} \\
& =1-q^{2} \pi \int_{0}^{\pi} d \theta \sin \theta \cos ^{2} \theta \int_{0}^{\infty} d u u^{4} P_{1}(u) \\
& =1-q^{2} \frac{1}{6} \int_{0}^{\infty} d u 4 \pi u^{2} P_{1}(u) u^{2}=1-\frac{1}{6}\left\langle u^{2}\right\rangle q^{2} \\
& \cong \exp \left[-\frac{1}{6}\left\langle u^{2}\right\rangle q^{2}\right]
\end{aligned}
$$

where $q$ denotes $|\boldsymbol{q}|$ and $\left\langle u^{2}\right\rangle$ the mean square of $u$. The length $u_{s}:=\sqrt{\left\langle u^{2}\right\rangle}$ corresponds to the segment size.

By substituting (A.7) for eq. (A.2) and performing the inverse Fourier transformation in the spherical coordinate system of $\boldsymbol{q}$,

$$
\begin{aligned}
P(\boldsymbol{R}) & =\frac{1}{(2 \pi)^{3}} \int d \boldsymbol{q} \exp [-i \boldsymbol{q} \cdot \boldsymbol{R}] \exp \left[-\frac{1}{6}(N-1)\left\langle u^{2}\right\rangle q^{2}\right] \\
& =\frac{1}{(2 \pi)^{2}} \int_{0}^{\infty} d q \int_{0}^{\pi} d \theta \exp [-i q R \cos \theta] \exp \left[-a q^{2}\right] q^{2} \sin \theta \\
& =-\frac{1}{(2 \pi)^{2}} \frac{1}{i R} \exp \left[-\frac{R^{2}}{4 a}\right]\left(J_{1}-J_{2}\right),
\end{aligned}
$$

where $R$ denotes $|\boldsymbol{R}|$ and

$$
\begin{gathered}
a:=\frac{1}{6}(N-1)\left\langle u^{2}\right\rangle, \\
J_{1}:=\int_{0}^{\infty} d q q \exp \left[-a\left(q+\frac{i R}{2 a}\right)^{2}\right] \\
J_{2}:=\int_{0}^{\infty} d q q \exp \left[-a\left(q-\frac{i R}{2 a}\right)^{2}\right] .
\end{gathered}
$$


Integrals $J_{1}$ and $J_{2}$ are calculated on the complex plane with $z:=x+i y$.

$$
\begin{aligned}
& J_{1}=\lim _{x_{0} \rightarrow \infty} \int_{C_{0}} d z f_{1}(z)=\lim _{x_{0} \rightarrow \infty} \int_{C_{1}+C_{2}+C_{3}} d z f_{1}(z) \\
& J_{2}=\lim _{x_{0} \rightarrow \infty} \int_{C_{0}} d z f_{2}(z)=\lim _{x_{0} \rightarrow \infty} \int_{C_{4}+C_{5}+C_{6}} d z f_{2}(z),
\end{aligned}
$$

where the straight integration paths $C_{0}, C_{1}, \ldots, C_{6}$ are shown in Table. A.1 and Fig. A.7, and

$$
\begin{aligned}
& f_{1}(z):=z \exp \left[-a\left(z+\frac{i R}{2 a}\right)^{2}\right] \\
& f_{2}(z):=z \exp \left[-a\left(z-\frac{i R}{2 a}\right)^{2}\right] .
\end{aligned}
$$

\begin{tabular}{|c|c|c|c|c|c|c|c|}
\hline$C_{0}$ & $(0,0)$ & $\rightarrow$ & $\left(x_{0}, 0\right)$ & & & & \\
\hline$C_{1}$ & $(0,0)$ & $\rightarrow$ & $(0,-R / 2 a)$ & $C_{4}$ & $(0,0)$ & $\rightarrow$ & $(0, R / 2 a)$ \\
\hline$C_{2}$ & $(0,-R / 2 a)$ & $\rightarrow$ & $\left(x_{0},-R / 2 a\right)$ & $C_{5}$ & $(0, R / 2 a)$ & $\rightarrow$ & $\left(x_{0}, R / 2 a\right)$ \\
\hline$C_{3}$ & $\left(x_{0},-R / 2 a\right)$ & $\rightarrow$ & $\left(x_{0}, 0\right)$ & $C_{6}$ & $\left(x_{0}, R / 2 a\right)$ & $\rightarrow$ & $\left(x_{0}, 0\right)$ \\
\hline
\end{tabular}

Table A.1: Straight integration paths on the complex plane.

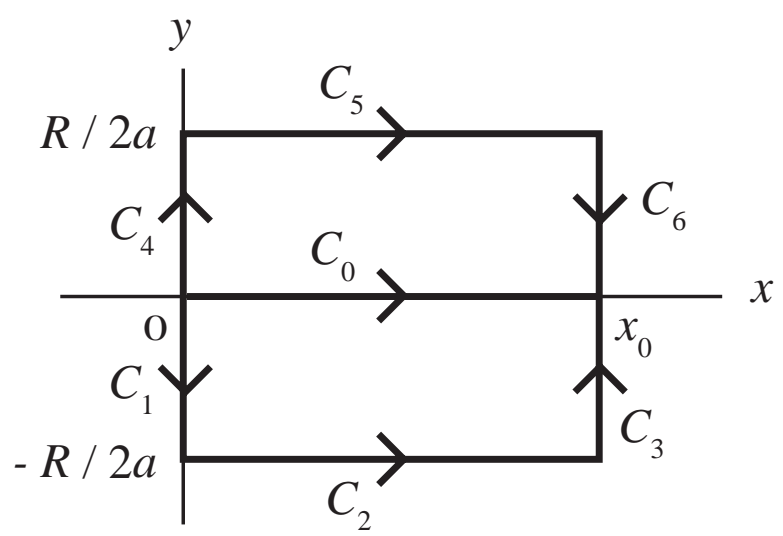

Figure A.7: Straight integration paths on the complex plane.

These complex integrals are calculated on each path.

$$
\begin{gathered}
\int_{C_{1}} d z f_{1}(z)=\int_{-R / 2 a}^{0} d y y \exp \left[a\left(y+\frac{R}{2 a}\right)^{2}\right] \\
\int_{C_{4}} d z f_{2}(z)=-\int_{0}^{R / 2 a} d y y \exp \left[a\left(y-\frac{R}{2 a}\right)^{2}\right] \\
\int_{C_{1}} d z f_{1}(z)-\int_{C_{4}} d z f_{2}(z)=0 .
\end{gathered}
$$


On the paths $C_{3}$ and $C_{6}$, the integrands vanish in the limit $x_{0} \rightarrow \infty$.

$$
\lim _{x_{0} \rightarrow \infty} \int_{C_{3}} d z f_{1}(z)=\lim _{x_{0} \rightarrow \infty} \int_{C_{6}} d z f_{2}(z)=0
$$

On the paths $C_{2}$ and $C_{5}$,

$$
\begin{aligned}
& \int_{C_{2}} d z f_{1}(z)=\int_{0}^{x_{0}} d x\left(x-\frac{i R}{2 a}\right) \exp \left[-a x^{2}\right] \\
& \int_{C_{5}} d z f_{2}(z)=\int_{0}^{x_{0}} d x\left(x+\frac{i R}{2 a}\right) \exp \left[-a x^{2}\right] .
\end{aligned}
$$

These results yield the relation

$$
J_{1}-J_{2}=-\frac{R i}{a} \int_{0}^{\infty} d x \exp \left[-a x^{2}\right]=-\frac{R i}{2 a} \sqrt{\frac{\pi}{a}} .
$$

Therefore, eq. (A.8) is:

$$
P(\boldsymbol{R})=\left(\frac{3}{2 \pi N_{\text {bond }}\left\langle u^{2}\right\rangle}\right)^{3 / 2} \exp \left[-\frac{3|\boldsymbol{R}|^{2}}{2 N_{\text {bond }}\left\langle u^{2}\right\rangle}\right]=\text { eq. (A.3). }
$$

The probability density of each bond vector is also calculated by the inverse Fourier transformation,

$$
\begin{aligned}
P_{1}(u) & =\frac{1}{(2 \pi)^{3}} \int d \boldsymbol{q} \exp [-i \boldsymbol{q} \cdot \boldsymbol{u}] \hat{P}_{1}(\boldsymbol{q}) \\
& =\frac{1}{(2 \pi)^{3}} \int d \boldsymbol{q} \exp [-i \boldsymbol{q} \cdot \boldsymbol{u}] \exp \left[-\frac{1}{6}\left\langle u^{2}\right\rangle q^{2}\right] \\
& =\left(\frac{3}{2 \pi\left\langle u^{2}\right\rangle}\right)^{3 / 2} \exp \left[-\frac{3 u^{2}}{2\left\langle u^{2}\right\rangle}\right],
\end{aligned}
$$

and

$$
P_{N_{\text {bond }}}\left(\boldsymbol{u}_{1}, \ldots, \boldsymbol{u}_{N-1}\right)=\left(\frac{3}{2 \pi\left\langle u^{2}\right\rangle}\right)^{\frac{3}{2} N_{\text {bond }}} \exp \left[-\frac{3}{2\left\langle u^{2}\right\rangle} \sum_{j=1}^{N_{\text {bond }}}\left|\boldsymbol{u}_{j}\right|^{2}\right]=\text { eq. (A.4). }
$$




\section{Appendix B}

\section{Particle deletion}

In the configurational-bias method, to remove a molecule from the system, we use the following 4-stage algorithm. In the particle deletion, the molecule is removed with the same scheme as the insertion.

1) A molecule is randomly selected. The non-bonded potential energy between the first atom, the end segment, and the other molecules is calculated and is defined as $u^{0, \mathrm{nb}}(1)$. None of the other segments in the chosen molecule is taken into account in $u^{0, \mathrm{nb}}(1)$.

2) For the $l$-th segment, the non-bonded potential energy between this segment and other segments i.e. the other molecules and the segments from the 1st to the $(l-1)$-th in the chosen molecule, denoted by $u_{l}^{0, \mathrm{nb}}(l)$, is calculated. A set of $k-1$ trial orientations is generated with a probability given by eq. (3.28), in which the internal potential energy is determined without the $l$-th to the $M$-th segments. Using this set of trial positions and the actual position, we calculate for the $l$-th segment

$$
w^{0, n b}(l)=\exp \left[-\beta u_{l}^{0, n b}(l)\right]+\sum_{j=2}^{k} \exp \left[-\beta u_{l}^{0, n b}\left(\boldsymbol{b}_{j}^{l}\right)\right],
$$

where $u_{l}^{0, n b}\left(\boldsymbol{b}_{j}^{l}\right)$ denotes the non-bonded potential energy between the trial position as the $l$-th segment and other segments i.e. the other molecules and the segments from the 1 st to the $(l-1)$-th in the chosen molecule. This step 2$)$ is also schematically shown in Fig. B.1.

3) After repeating step 2) until all $M$ atoms of the molecule have been considered, we calculate the Rosenbluth weight

$$
\mathcal{W}^{0}:=\exp \left[-\beta u^{0, n b}(1)\right] \prod_{l=2}^{M} \frac{w^{0, n b}(l)}{k}
$$

for the entire molecule. 
4) The chosen molecule is removed with a probability of

$$
\operatorname{acc}(N \rightarrow N-1 \mid\{\boldsymbol{B}\})=\min \left(1, \frac{\Lambda^{3} N}{V \exp [\beta \mu} \frac{1}{\mathcal{W}^{0}} \frac{1}{j(T)}\right) .
$$

With use of $\rho_{\text {res }}$, this formula is reduced to

$$
\operatorname{acc}(N \rightarrow N-1 \mid\{\boldsymbol{B}\})=\min \left(1, \frac{N}{V} \frac{1}{\rho_{\text {res }}} \frac{1}{\mathcal{W}^{0}}\right) .
$$

Thus, $j(T)$ and $\Lambda$ disappear from the present algorithm. Regardless of these two quantities, we can simulate our systems.

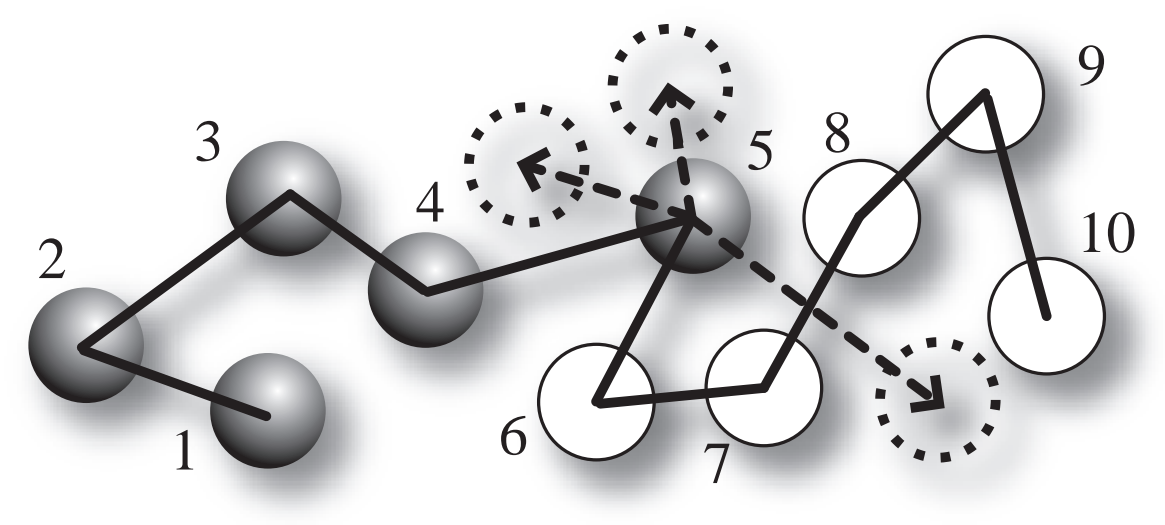

Figure B.1: Step 2) in the particle deletion at $l=6, k=4$, and $M=10$. As an example, the inserted molecule in Fig. 3.2 is deleted. The numbers in the present figure show the segment numbers. White spheres represent the real segments which are not taken into accout in the energy calculation. First, the non-bonded potential energy between the $l$-th segment and the other segments in the system excluding the white ones is determined. $k-1$ trial positions are generated according to the internal potential energy between each trial position and the 1 st to the $(l-1)$-th segments. For the trial positions, the non-bonded potential energy is also calculated between each of the trial 6th segment and the other segments in the system excluding the white ones. 


\section{Bibliography}

[1] Yuki Norizoe, Kostas Ch. Daoulas, and Marcus Müller. Measuring excess free energies of self-assembled membrane structures. Faraday Discussions, 144:369-391, 2010.

[2] Bermudez H, Brannan AK, Hammer DA, Bates FS, and Discher DE. Macromolecules, 35:8203, 2002.

[3] B. M. Discher, Y. Won, D. S. Ege, JC-M. Lee, F. S. Bates, D. E. Discher, and D. A. Hammer. Science, 284:1143, 1999.

[4] D. E. Discher and A. Eisenberg. Science, 297:967, 2002.

[5] I. W. Hamley. Soft Matter, 1:36, 2005.

[6] L. Luo and A. Eisenberg. Langmuir, 17:6804, 2001.

[7] M. Müller, K. Katsov, and M. Schick. J. Polym. Sci. B Polym. Phys. B, 41:1441, 2003.

[8] J. M. Drouffe, A. C. Maggs, and S. Leibler. Computer-simulations of selfassembled membranes. SCIENCE, 254(5036):1353 - 1356, 1991.

[9] H. Noguchi and M. P. Takasu. Phys. Rev. E, 64:041913, 2001.

[10] O. Farago. J. Chem. Phys., 119:596, 2003.

[11] I. R. Cooke, K. Kremer, Deserno, and M. Phys. Rev. E, 72:011506, 2005.

[12] I. R. Cooke and M. Deserno. J. Chem. Phys., 123:224710, 2005.

[13] Kostas Ch. Daoulas and Marcus Müller. Adv. Polym. Sci., 224:197-233, 2009.

[14] M. Laradji, H. Guo, and M. J. Zuckermann. J. Phys. Rev. E, 49:3199, 1994.

[15] K. G. Soga, M. J. Zuckermann, and H. Guo. Macromolecules, 29:2289, 1996.

[16] L. Miao, H. Guo, and M. J. Zuckermann. Macromolecules, 29:2289, 1996. 
[17] Kostas Ch. Daoulas and Marcus Müller. Single chain in mean field simulations: Quasi-instantaneous field approximation and quantitative comparison with monte carlo simulations. The Journal of Chemical Physics, 125(18):184904, 2006.

[18] P. Espanol and P. B. Warren. Europhys. Lett., 30:191, 1995.

[19] R. D. Groot and P. B. Warren. J. Chem. Phys., 107:4423, 1997.

[20] J. C. Shillcock and R. Lipowsky. Equilibrium structure and lateral stress distribution of amphiphilic bilayers from dissipative particle dynamics simulations. JOURNAL OF CHEMICAL PHYSICS, 117(10):5048 - 5061, 2002.

[21] A Mayer. Membrane fusion in eukaryotic cells. ANNUAL REVIEW OF CELL AND DEVELOPMENTAL BIOLOGY, 18:289-314, 2002.

[22] Lukas K. Tamm, Jonathan Crane, and Volker Kiessling. Membrane fusion: a structural perspective on the interplay of lipids and proteins. Current Opinion in Structural Biology, 13(4):453 - 466, 2003.

[23] R Blumenthal, MJ Clague, SR Durell, and RM Epand. Membrane fusion. CHEMICAL REVIEWS, 103(1):53-69, JAN 2003.

[24] J. Zimmerberg and L. V. Chernomordik. Synaptic membranes bend to the wilt of a neurotoxin. SCIENCE, 310(5754):1626-1627, DEC 92005.

[25] Axel T. Brunger. Structure and function of snare and snare-interacting proteins. Quarterly Reviews of Biophysics, 38(01):1-47, 2005.

[26] Leonid V. Chernomordik and Michael M. Kozlov. Mechanics of membrane fusion. NATURE STRUCTURAL E MOLECULAR BIOLOGY, 15(7):675683, JUL 2008.

[27] Lin Yang and Huey W. Huang. Observation of a Membrane Fusion Intermediate Structure. Science, 297(5588):1877-1879, 2002.

[28] S. Aeffner, T. Reusch, B. Weinhausen, and T. Salditt. The European Physical Journal E: Soft Matter and Biological Physics, 30:205-214, 2009.

[29] Y. L. Loo, R. A. Register, D. H. Adamson, and A. J. Ryan. A highly regular hexagonally perforated lamellar structure in a quiescent diblock copolymer. MACROMOLECULES, 38(11):4947-4949, MAY 312005.

[30] K Katsov, M Müller, and M Schick. Field theoretic study of bilayer membrane fusion. I. Hemifusion mechanism. BIOPHYSICAL JOURNAL, 87(5):3277-3290, NOV 2004.

[31] K Katsov, M Müller, and M Schick. Field theoretic study of bilayer membrane fusion: II. Mechanism of a stalk-hole complex. BIOPHYSICAL JOURNAL, 90(3):915-926, FEB 2006. 
[32] Y. Kozlovsky and M. M. Kozlov. Stalk model of membrane fusion: Solution of energy crisis. BIOPHYSICAL JOURNAL, 82(2):882-895, FEB 2002.

[33] M. Müller and J. J. de Pablo. Lec. Notes Phys., 703:67, 2006.

[34] Daan Frenkel and Anthony J. C. Ladd. New monte carlo method to compute the free energy of arbitrary solids. application to the fcc and hcp phases of hard spheres. The Journal of Chemical Physics, 81(7):3188-3193, 1984.

[35] E. M. Lennon, K. Katsov, and G. H. Fredrickson. Phys. Rev. Lett., 101:138302, 2002.

[36] Marcus Müller and Kostas Ch. Daoulas. Calculating the free energy of selfassembled structures by thermodynamic integration. The Journal of Chemical Physics, 128(2):024903, 2008.

[37] Marcus Müller, Kostas Ch. Daoulas, and Yuki Norizoe. Computing free energies of interfaces in self-assembling systems. PHYSICAL CHEMISTRY CHEMICAL PHYSICS, 11(12):2087-2097, 2009.

[38] Sheh-Yi Sheu, Chung-Yuan Mou, and Ronald Lovett. How a solid can be turned into a gas without passing through a first-order phase transformation. Phys. Rev. E, 51(5):R3795-R3798, May 1995.

[39] G. Grochola. J. Chem. Phys., 120:2122, 2004.

[40] D. M. Eike, J. F. Brennecke, and E. J. Maginn. J. Chem. Phys., 122:014115, 2005.

[41] J. W. Eastwood, R. W. Hockney, and D. N. Lawrence. P3m3dp - the 3dimensional periodic particle-particle-particle-mesh program. COMPUTER PHYSICS COMMUNICATIONS, 19(2):215 - 261, 1980.

[42] John M. Dawson. Particle simulation of plasmas. Rev. Mod. Phys., 55(2):403-447, Apr 1983.

[43] Markus Deserno and Christian Holm. How to mesh up ewald sums. i. a theoretical and numerical comparison of various particle mesh routines. The Journal of Chemical Physics, 109(18):7678-7693, 1998.

[44] G. Brannigan and F. L. H. Brown. Solvent-free simulations of fluid membrane bilayers. JOURNAL OF CHEMICAL PHYSICS, 120(2):1059 - 1071, 2004.

[45] Pierre giles De Gennes. Scaling Concepts in Polymer Physics. Cornell Univ Press, 1979.

[46] Glenn H. Fredrickson and Eugene Helfand. Fluctuation effects in the theory of microphase separation in block copolymers. The Journal of Chemical Physics, 87(1):697-705, 1987. 
[47] M. Rovere, D. W. Heermann, and K. Binder. The gas-liquid transition of the 2-dimensional lennard-jones fluid. JOURNAL OF PHYSICS-CONDENSED MATTER, 2(33):7009 - 7032, 1990.

[48] Akira Ueda. Molecular Simulation - from Classical to Quantum Methods -. Shokabo Publishing, Tokyo, 2003.

[49] M. E. Cates and M. R. Evans, editors. Soft and Fragile Matter. Taylor \& Francis, 2000.

[50] Daan Frenkel and Berend Smit. Understanding Molecular Simulation. Academic Press, 1996.

[51] M. Matsumoto and T. Nishimura. ACM Trans. on Modeling and Computer Simulation, 8:3, 1998.

[52] M. Matsumoto and Y. Kurita. ACM Trans. on Modeling and Computer Simulation, 2:179, 1992.

[53] M. Matsumoto and Y. Kurita. ACM Trans. on Modeling and Computer Simulation, 4:254, 1994.

[54] B. Smit. Grand-canonical monte-carlo simulations of chain molecules - adsorption-isotherms of alkanes in zeolites. MOLECULAR PHYSICS, 85(1):153 - 172, 1995.

[55] B. Widom. Some topics in the theory of fluids. The Journal of Chemical Physics, 39(11):2808-2812, 1963.

[56] Charles H. Bennett. Efficient estimation of free energy differences from monte carlo data. Journal of Computational Physics, 22(2):245 - 268, 1976.

[57] K. S. Shing and K. E. Gubbins. The chemical potential in dense fluids and fluid mixtures via computer simulation. Molecular Physics, 46(5):11091128, 1982.

[58] Marshall N. Rosenbluth and Arianna W. Rosenbluth. Monte carlo calculation of the average extension of molecular chains. The Journal of Chemical Physics, 23(2):356-359, 1955.

[59] D. Frenkel, G. C. A. M. Mooij, and B. Smit. Novel scheme to study structural and thermal properties of continuously deformable molecules. Journal of Physics: Condensed Matter, 4(12):3053-3076, 1992.

[60] A. P. Lyubartsev, A. A. Martsinovski, S. V. Shevkunov, and P. N. VorontsovVelyaminov. New approach to monte carlo calculation of the free energy: Method of expanded ensembles. The Journal of Chemical Physics, 96(3):1776-1783, 1992. 
[61] D. A. Kofke and E. D. Glandt. MONTE-CARLO SIMULATION OF MULTICOMPONENT EQUILIBRIA IN A SEMIGRAND CANONICAL ENSEMBLE. Molecular Physics, 64(6):1105-1131, AUG 201988.

[62] S. Sengupta, D. Marx, P. Nielaba, and K. Binder. Phase diagram of a model anticlustering binary mixture in two dimensions: A semi-grand-canonical monte carlo study. Phys. Rev. E, 49(2):1468-1477, Feb 1994.

[63] K. S. Shing. Infinite-dilution activity coefficients of quadrupolar lennardjones mixtures from computer simulation. The Journal of Chemical Physics, 85(8):4633-4637, 1986.

[64] J. F. Nagle and S. Tristram-Nagle. Structure of lipid bilayers. Biochimica et Biophysica Acta-Reviews on Biomembranes, 1469(3):159 - 195, 2000.

[65] Alan M. Ferrenberg and Robert H. Swendsen. Optimized monte carlo data analysis. Phys. Rev. Lett., 63(12):1195-1198, Sep 1989.

[66] S. Kumar, J. M. Rosenberg, D. Bouzida, R. H. Swendsen, and P. A. Kollman. The weighted histogram analysis method for free-energy calculations on biomolecules. I. The method. Journal of Computational Chemistry, 13(8):1011-1021, 1992.

[67] Mark J. Stevens, Jan H. Hoh, and Thomas B. Woolf. Insights into the molecular mechanism of membrane fusion from simulation: Evidence for the association of splayed tails. Phys. Rev. Lett., 91(18):188102, Oct 2003.

[68] Alexander Yu Grosberg and Alexei R. Khokhlov. Statistical Physics of Macromolecules. American Institute of Physics, 1994.

[69] Gert R. Strobl. The Physics of Polymers. Springer, 1997.

[70] T. Kawakatsu. Statistical physics of polymers: an introduction. Springer, 2004. 
Doctoral thesis

\section{Yuki Norizoe}

Göttingen

Defense on January 21 (Thursday), 2010 


\section{Acknowledgements}

This challenging work has been accomplished with the help of quite a few people in Göttingen. The author would like to, first, thank Prof. Dr Marcus Müller, the author's supervisor. The present research project started when he offered me a $\mathrm{PhD}$ student position in his group, the theoretical physics institute at Göttingen university. He always encouraged and led me to this goal during the doctoral program.

Since the author came to Germany from Japan, Dr Kostas Ch. Daoulas gave me a lot of invaluable advice. Without his suggestions and scientific discussions, this project could not be completed. The author appreciates his great help.

I also wish to thank Mr Martin Hömberg in our research group. His generous character relieved my insecurity in the first long overseas life.

Finally, I would like to thank the other people in our group, in our institute, and in Göttingen. Especially, Traude Heinrich, who was the landlady where I stayed for the first one month in Göttingen, came to my aid, when I seriously got sick just after moving from her house to a studio apartment. Japanese people in Göttingen and in Japan also encouraged and supported me. I would like to express to them my gratitude. 


\section{Zusammenfassung}

Messung der Freien Energie von spontan strukturbildenden Systemen mittels Computersimulation. 


\section{Erklärung}

Ich erkläre an Eides statt dass die Dissertation mit dem Titel "Measuring the Free Energy of Self-assembling Systems in Computer Simulation" selbständig und ohne unerlaubte Hilfe angefertigt wurde.

Göttingen

Datum:

Yuki Norizoe 


\section{Erklärung}

Ich erkläre,

1) dass ich mich an keiner anderen Universität um einen Doktorgrad beworben habe,

2) dass 4 Jahre für die Promotion (ab dem Diplom bis zur Promotion) studiert wurden.

Göttingen

Datum:

Yuki Norizoe 


\section{Lebenslauf}

\section{Persönlich}

$\begin{array}{ll}\text { Name: } & \text { Yuki Norizoe } \\ \text { Geburtsdatum: } & \text { Dezember 27, 1977 } \\ \text { Geburtsort: } & \text { Tokyo, Japan } \\ \text { Staatsangehörigkeit: } & \text { Japanisch } \\ \text { Sprachen: } & \text { Japanisch, Englisch }\end{array}$

\section{Ausbildung}

4/1993 - 3/1996 Seibu Gakuen Bunri High School, Sayama, Saitama (Japan).

4/1997 - 3/2001 Bachelor of Science, Fakultät für Physik,

Gakushuin Universität, Tokyo (Japan).

4/2002 - 3/2004 Master of Science, Fakultät für Physik,

Tohoku Universität, Sendai (Japan).

Thema:

4/2006 -

Monte-Carlo simulation of string-like colloidal assembly

Doktorarbeit, Institut für Theoretische Physik,

Georg-August-Universität zu Göttingen.

Betreuer: Prof. Dr. M. Müller

Göttingen

Datum:

Yuki Norizoe 